\title{
CELL CENTERED GALERKIN METHODS FOR DIFFUSIVE PROBLEMS*
}

\author{
Daniele A. Di Pietro ${ }^{1}$
}

\begin{abstract}
In this work we introduce a new class of lowest order methods for diffusive problems on general meshes with only one unknown per element. The underlying idea is to construct an incomplete piecewise affine polynomial space with optimal approximation properties starting from values at cell centers. To do so we borrow ideas from multi-point finite volume methods, although we use them in a rather different context. The incomplete polynomial space replaces classical complete polynomial spaces in discrete formulations inspired by discontinuous Galerkin methods. Two problems are studied in this work: a heterogeneous anisotropic diffusion problem, which is used to lay the pillars of the method, and the incompressible Navier-Stokes equations, which provide a more realistic application. An exhaustive theoretical study as well as a set of numerical examples featuring different difficulties are provided.
\end{abstract}

Mathematics Subject Classification. 65N08, 65N30, 76D05

Received August 23, 2010. Revised December 13, 2010.

Published online August 24, 2011.

\section{Introduction}

Lowest order methods for diffusive problems on general meshes have received an increasing attention over the last few years. The interest of general meshes is multi-fold. On the one hand, allowing general polyhedral elements may ease the discretization of complex domains, and is beneficial in the context of aggregative multigrid strategies. On the other hand, it is a mandatory requirement whenever the user cannot adapt the mesh to the needs of the numerical scheme. This is the case, e.g., in the context of computational geosciences, where the discretization of the subsoil integrates the effects of erosion and sedimentation, and is usually developed in a separate stage. In what follows we briefly recall some facts that are instrumental to the class of methods proposed in this paper. It is a well known fact that the classical two-point finite volume (FV) method is inconsistent on non diffusion-orthogonal meshes. In the context of reservoir simulation, a successful attempt to adapt FV methods to general meshes and full diffusion tensors has been independently proposed in the 90s by Aavatsmark et al. [1,2] and by Edwards and Rogers [28,29]. These methods are usually referred to as multi-point since the main idea is to express consistent numerical fluxes using a larger stencil than in the classical two-point FV method. In this paper we borrow some ideas from the recent multi-point scheme of

\footnotetext{
Keywords and phrases. Cell centered Galerkin, finite volumes, discontinuous Galerkin, heterogeneous anisotropic diffusion, incompressible Navier-Stokes equations.

* This work has been partially supported by the VFSitCom ANR project (ANR-08-BLAN-0275-01) and by GDR MoMaS TC5 project.

${ }^{1}$ IFP Energies nouvelles, 1 \& 4 avenue de Bois Préau, 92582 Rueil-Malmaison Cedex, France.

daniale-antonio.di-pietro@ifpen.fr
} 
Aavatsmark et al. [3], where the authors propose a compact construction to derive a consistent multi-point flux approximation honoring the heterogeneity of the diffusion tensor. A convergence analysis of the $L$-construction proposed therein has been recently carried out by Agélas et al. [4]. The main drawback of multi-point FV methods is related to the difficulty in finding easily computable stability conditions. A possible remedy to the lack of stability in multi-point methods has been proposed independently by Brezzi et al. [11,12] (mimetic finite difference methods, MFD) and by Eymard et al. [26,33] (mixed/hybrid finite volume methods, MHFV). Both classes of schemes are inspired from the variational form of the problem as in finite element (FE) methods rather than from local balances as in FV methods. The unconditional stability of both MFD and MHFV methods results from stabilization terms incorporated in the discrete bilinear form. Also, in both cases auxiliary face unknowns are added, which can be in some cases related to the enforcement of a flux conservation constraint as in the mixed FE method. A way to eliminate face unknowns has been proposed in [33], where it is suggested that a barycentric interpolator can be used to express face values in terms of a few neighboring cell unknowns. The main drawback of this construction is that it does not respect the heterogeneity of the diffusion tensor; moreover, the resulting method has a larger stencil compared to multi-point FV methods, which results in denser matrices as well as in the increase of data exchange in parallel implementations. The algebraic analogy between generalized versions of the MFD and MHFV methods has been recently pointed out by Droniou et al. [27].

In this work we propose a different philosophical approach to variational lowest-order methods which is inspired by discontinuous Galerkin $(\mathrm{dG})$ methods rather than mixed FEs. The motivation is twofold. A first objective is to devise a suitable framework for a multi-physics platform based on lowest-order methods. In this respect, the main requirement is to dispose of a method easily adapted to a variety of diffusive problems including, e.g., pure diffusion, diffusion-advection-reaction, linear and nonlinear elasticity as well as incompressible flows. A second important point is related to the robustness in the context of heterogeneous anisotropic diffusion, which is crucial in the field of computational geosciences. Cell centered Galerkin (ccG) methods have been introduced in [20,21] with application to a homogeneous diffusion problem. The main idea of ccG methods is to build an incomplete space of piecewise affine functions related to a given vector of degrees of freedom (DOFs) on the mesh $\mathcal{T}_{h}$. In this work we consider, in particular, ccG methods with cell unknowns only, i.e., the vector space of DOFs given by

$$
\mathbb{V}_{h}:=\mathbb{R}^{\mathcal{T}_{h}}
$$

The unknown in each mesh element $T \in \mathcal{T}_{h}$ is interpreted as the value of the discrete function at a given point $\mathbf{x}_{T}$ (the cell center). For $k \geq 0$ we define the space of broken polynomials on $\mathcal{T}_{h}$ of degree $\leq k$,

$$
\mathbb{P}_{d}^{k}\left(\mathcal{T}_{h}\right):=\left\{v \in L^{2}(\Omega) \mid \forall T \in \mathcal{T}_{h}, v_{\mid T} \in \mathbb{P}_{d}^{k}(T)\right\}
$$

with $\mathbb{P}_{d}^{k}(T)$ given by the restriction to $T$ of the functions in $\mathbb{P}_{d}^{k}$. A piecewise affine representation is then obtained by reconstructing a piecewise constant gradient on $\mathcal{T}_{h}$, say $\mathfrak{G}_{h}: \mathbb{V}_{h} \rightarrow\left[\mathbb{P}_{d}^{0}\left(\mathcal{T}_{h}\right)\right]^{d}$, and introducing the linear operator $\mathfrak{R}_{h}^{\mathrm{ccg}}: \mathbb{V}_{h} \rightarrow \mathbb{P}_{d}^{1}\left(\mathcal{T}_{h}\right)$ which maps every vector of degrees of freedom $\mathbf{v}_{h} \in \mathbb{V}_{h}$ onto $\mathfrak{R}_{h}\left(\mathbf{v}_{h}\right) \in \mathbb{P}_{d}^{1}\left(\mathcal{T}_{h}\right)$ such that

$$
\forall T \in \mathcal{T}_{h}, \forall \mathbf{x} \in T, \quad \mathfrak{R}_{h}\left(\mathbf{v}_{h}\right)_{\mid T}(\mathbf{x})=v_{T}+\mathfrak{G}_{h}\left(\mathbf{v}_{h}\right)_{\mid T} \cdot\left(\mathbf{x}-\mathbf{x}_{T}\right) .
$$

In the present work, the elementary gradient is obtained in two steps: first, trace values are reconstructed from values at cell centers by means of the L-construction, then Green's formula is used to infer a local value for the gradient. Formally, this procedure amounts to defining the discrete space

$$
V_{h}^{\mathrm{ccg}}:=\mathfrak{R}_{h}^{\mathrm{ccg}}\left(\mathbb{V}_{h}\right) \subset \mathbb{P}_{d}^{1}\left(\mathcal{T}_{h}\right) .
$$

The space $V_{h}^{\text {ccg }}$ is then used as a test/trial space in a suitable nonconforming FE setting. In particular, since the functions in $V_{h}^{\mathrm{ccg}}$ are discontinuous across mesh interfaces, the discrete setting largely borrows from $\mathrm{dG}$ methods. We consider two applications to problems naturally set in $H^{1}(\Omega)$ : a homogeneous anisotropic scalar diffusion problem and the incompressible Navier-Stokes equations. The scalar diffusion problem offers a simplified context to outline the main ideas of the method. The convergence analysis is here discussed in detail. In particular, we show that both classical dG arguments relying on error estimates [7] and compactness 
arguments inspired by [32,33] apply. For the latter point, a key remark is that the functional analytic results for broken polynomial spaces derived independently by Di Pietro and Ern [22] and Buffa and Ortner [14] hold a fortiori since $V_{h}^{\mathrm{ccg}} \subset \mathbb{P}_{d}^{1}\left(\mathcal{T}_{h}\right)$. In the context of $h$-convergence analysis the main technical issue is related to the approximation properties of the $V_{h}^{\mathrm{ccg}}$ space. The application to the incompressible Navier-Stokes equations provides a nonlinear case study to illustrate how a ccG method can be derived from an existing dG method.

The material is organized as follows: in Section 2 we discuss the discrete setting, provide a careful description of admissible mesh sequences in arbitrary space dimension, introduce the L-construction, and define the ccG space used throughout the rest of this work. Some functional analytical results are also recalled; in Section 3 we show an application of the ccG method to a heterogeneous anisotropic diffusion problem, derive basic error estimates and carefully study convergence rates. The main sources of inspiration are here the work of Arnold [6] on the weak enforcement of boundary and interface conditions and that of Di Pietro et al. [25] on the robust handling of heterogeneous anisotropic diffusion tensors. To infer convergence rates we study the approximation properties of the space $V_{h}^{\text {ccg }}$ with respect to the energy norm naturally associated to the discrete problem. In particular, we distinguish between the heterogeneous case, where optimal energy estimates are obtained for functions belonging to the space introduced and analyzed in [4], and the homogeneous case, where weaker regularity assumptions are sufficient and $L^{2}$-error estimates can also be derived; in Section 4 we discuss the application of ccG methods to the incompressible Navier-Stokes equations inspired from the dG scheme of [22]. In this case, a $H^{1}$-stability result for ccG spaces is crucial to infer the stability of the velocity-pressure coupling. The analysis closely follows the guidelines of [22]; finally, in Section 5 we present numerical results to assess the theoretical study for both problems at hand.

\section{THE CCG SPACE}

\subsection{Discrete setting}

Closely following [24], Chapter 1, we introduce the concept of admissible mesh sequence of a bounded connected polyhedral domain $\Omega \subset \mathbb{R}^{d}, d \geq 2$. Let $\mathcal{H} \subset \mathbb{R}_{+}^{*}$ denote a countable set having 0 as its unique accumulation point. For all $h \in \mathcal{H}$ we denote by $\mathcal{T}_{h}$ a finite collection of nonempty, disjoint open polyhedra $\mathcal{T}_{h}=\{T\}$ forming a partition of $\Omega$ such that $h=\max _{T \in \mathcal{T}_{h}} h_{T}$, with $h_{T}$ denoting the diameter of the element $T \in \mathcal{T}_{h}$. Mesh nodes are collected in the set $\mathcal{N}_{h}$. We say that a hyperplanar closed subset $F$ of $\bar{\Omega}$ is a mesh face if it has positive $(d-1)$-dimensional measure and if either there exist $T_{1}, T_{2} \in \mathcal{T}_{h}$ such that $F \subset \partial T_{1} \cap \partial T_{2}$ (and $F$ is called an interface) or there exist $T \in \mathcal{T}_{h}$ such that $F \subset \partial T \cap \partial \Omega$ (and $F$ is called a boundary face). Interfaces are collected in the set $\mathcal{F}_{h}^{\mathrm{i}}$, boundary faces in $\mathcal{F}_{h}^{\mathrm{b}}$ and we let $\mathcal{F}_{h}:=\mathcal{F}_{h}^{\mathrm{i}} \cup \mathcal{F}_{h}^{\mathrm{b}}$. For a face $F \in \mathcal{F}_{h}$ we denote by $h_{F}$ its diameter. Moreover, we set, for all $T \in \mathcal{T}_{h}$,

$$
\mathcal{F}_{T}:=\left\{F \in \mathcal{F}_{h} \mid F \subset \partial T\right\}
$$

Similarly, for all $F \in \mathcal{F}_{h}$, we define

$$
\mathcal{T}_{F}:=\left\{T \in \mathcal{T}_{h} \mid F \subset \partial T\right\} .
$$

The set $\mathcal{T}_{F}$ consists of exactly two mesh elements if $F \in \mathcal{F}_{h}^{\mathrm{i}}$ and of one if $F \in \mathcal{F}_{h}^{\mathrm{b}}$. For all mesh nodes $P \in \mathcal{N}_{h}$ we define

$$
\mathcal{F}_{P}:=\left\{F \in \mathcal{F}_{h} \mid P \in F\right\}, \quad \mathcal{T}_{P}:=\left\{T \in \mathcal{T}_{h} \mid P \in \partial T\right\} .
$$

For every interface $F \in \mathcal{F}_{h}^{\mathrm{i}}$ we introduce an arbitrary but fixed ordering of the elements in $\mathcal{T}_{F}$ and let $\mathbf{n}_{F}=$ $\mathbf{n}_{T_{1}, F}=-\mathbf{n}_{T_{2}, F}$, where $\mathbf{n}_{T_{i}, F}, i \in\{1,2\}$, denotes the unit normal to $F$ pointing out $T_{i} \in \mathcal{T}_{F}$. For all $T \in \mathcal{T}_{h}$, we also introduce the symbol $\mathbf{n}_{T}$ to denote the vector field such that $\mathbf{n}_{T \mid F}=\mathbf{n}_{T, F}$ for all $F \in \mathcal{F}_{T}$. On a boundary face $F \in \mathcal{F}_{h}^{\mathrm{b}}$ we let $\mathbf{n}_{F}$ denote the unit normal pointing out of $\Omega$. The barycenter of a face $F \in \mathcal{F}_{h}$ is denoted by $\overline{\mathbf{x}}_{F}:=\int_{F} \mathbf{x} /|F|_{d-1}$.

Definition 2.1 (jumps and weighted averages). Let $v$ be a scalar-valued function defined on $\Omega$, and assume that $v$ is smooth enough to admit on all $F \in \mathcal{F}_{h}$ a possibly two-valued trace. To any interface $F \in \mathcal{F}_{h}^{\mathrm{i}}$ with 
$F \subset \partial T_{1} \cap \partial T_{2}$ we assign two non-negative real numbers $\omega_{T_{1}, F}$ and $\omega_{T_{2}, F}$ such that

$$
\omega_{T_{1}, F}+\omega_{T_{2}, F}=1
$$

Then, if $F \in \mathcal{F}_{h}^{\mathrm{i}}$ with $F=\partial T_{1} \cap \partial T_{2}$ the jump and weighted average of $v$ at $F$ are respectively defined for a.e. $\mathbf{x} \in F$ as

$$
\llbracket v \rrbracket_{F}(\mathbf{x}):=v_{\mid T_{1}}(\mathbf{x})-v_{\mid T_{2}}(\mathbf{x}), \quad\{v\}_{\omega, F}(\mathbf{x}):=\omega_{T_{1}, F} v_{\mid T_{1}}(\mathbf{x})+\omega_{T_{2}, F} v_{\mid T_{2}}(\mathbf{x}),
$$

while, if $F \in \mathcal{F}_{h}^{\mathrm{b}}$ with $F=\partial T \cap \partial \Omega$, we set $\{v\}_{\omega, F}(\mathbf{x})=\llbracket v \rrbracket_{F}(\mathbf{x})=v_{\mid T}(\mathbf{x})$.

When $v$ is vector-valued, the jump and average operators act component-wise. Whenever no confusion can arise, the subscript $F$ and the variable $\mathbf{x}$ are omitted, and we simply write $\{v\}_{\omega}$ and $\llbracket v \rrbracket$. Moreover, in the classical case $\omega_{T_{1}, F}=\omega_{T_{2}, F}=1 / 2$ we also omit the subscript $\omega$ and write $\{v\}$ instead of $\{v\}_{\omega}$. The use of weighted averages in $\mathrm{dG}$ methods has been pointed out and used in various contexts, e.g., by Stenberg [42] and by Heinrich and Pietsch [36].

Definition 2.2 (matching simplicial submesh). Let $\mathcal{T}_{h}$ be a general mesh. We say that $\mathfrak{S}_{h}$ is a matching simplicial submesh of $\mathcal{T}_{h}$ if (i) $\mathfrak{S}_{h}$ is a matching simplicial mesh, (ii) for all $T^{\prime} \in \mathfrak{S}_{h}$, there is only one $T \in \mathcal{T}_{h}$ such that $T^{\prime} \subset T$, (iii) for all $F^{\prime} \in \mathfrak{F}_{h}$, the set collecting the mesh faces of $\mathfrak{S}_{h}$, there is at most one $F \in \mathcal{F}_{h}$ such that $F^{\prime} \subset F$.

Definition 2.3 (shape- and contact-regularity). We say that the mesh sequence

$$
\mathcal{T}_{\mathcal{H}}:=\left(\mathcal{T}_{h}\right)_{h \in \mathcal{H}}
$$

is shape- and contact-regular if for all $h \in \mathcal{H}, \mathcal{T}_{h}$ admits a matching simplicial submesh $\mathfrak{S}_{h}$ such that (i) the mesh sequence $\left(\mathfrak{S}_{h}\right)_{h \in \mathcal{H}}$ is shape-regular in the usual sense of Ciarlet [18], meaning that there is a parameter $\varrho_{1}>0$ independent of $h$ such that for all $T^{\prime} \in \mathfrak{S}_{h}$,

$$
\varrho_{1} h_{T^{\prime}} \leq \delta_{T^{\prime}},
$$

where $h_{T^{\prime}}$ is the diameter of $T^{\prime}$ and $\delta_{T^{\prime}}$ the radius of the largest ball inscribed in $T^{\prime}$, (ii) there is a parameter $\varrho_{2}>0$ independent of $h$ such that for all $T \in \mathcal{T}_{h}$ and for all $T^{\prime} \in \mathfrak{S}_{T}$,

$$
\varrho_{2} h_{T} \leq h_{T^{\prime}}
$$

The following result is proven as in [24], Lemma 1.41.

Lemma 2.4 (bounds on geometric quantities). Let $\mathcal{T}_{\mathcal{H}}$ be a shape- and contact-regular mesh sequence. Then, for all $h \in \mathcal{H}$ and all $T \in \mathcal{T}_{h}$, (i) the number of mesh faces belonging to the boundary of an element is uniformly bounded in $h$,

(ii) for all $F \in \mathcal{F}_{T}$,

$$
N_{\partial}:=\sup _{h \in \mathcal{H}, T \in \mathcal{T}_{h}} \operatorname{card}\left(\mathcal{F}_{T}\right)<+\infty
$$

$$
h_{F} \geq \varrho_{1} \varrho_{2} h_{T} .
$$

For all $h \in \mathcal{H}$ and $k \geq 0$, we consider the broken polynomial spaces $\mathbb{P}_{d}^{k}\left(\mathcal{T}_{h}\right)$ defined by (1.1). Broken polynomial spaces are a special instance of broken Sobolev spaces: for all $k \geq 1$,

$$
H^{k}\left(\mathcal{T}_{h}\right):=\left\{v \in L^{2}(\Omega) \mid \forall T \in \mathcal{T}_{h}, v_{\mid T} \in H^{k}(T)\right\} .
$$

The shape- and contact-regularity of the mesh sequence $\mathcal{T}_{\mathcal{H}}$ are essential to infer the following results; see, e.g., [24], Lemmatas 1.46 and 1.49 . 
Lemma 2.5 (trace inequalities). Let $\mathcal{T}_{\mathcal{H}}$ be a shape- and contact-regular mesh sequence. Then, for all $h \in \mathcal{H}$, all $T \in \mathcal{T}_{h}$, and all $F \in \mathcal{F}_{T}$,

$$
\begin{array}{ll}
\forall v_{h} \in \mathbb{P}_{d}^{k}\left(\mathcal{T}_{h}\right), & \left\|v_{h}\right\|_{L^{2}(F)} \leq C_{\mathrm{tr}} h_{F}^{-1 / 2}\left\|v_{h}\right\|_{L^{2}(T)}, \\
\forall v \in H^{1}\left(\mathcal{T}_{h}\right), & \|v\|_{L^{2}(F)} \leq C_{\mathrm{tr}, \mathrm{c}}\left(h_{T}^{-1}\|v\|_{L^{2}(T)}^{2}+h_{T}|v|_{H^{1}(T)}^{2}\right)^{1 / 2},
\end{array}
$$

with $C_{\mathrm{tr}}$ and $C_{\mathrm{tr}, \mathrm{c}}$ independent of the meshsize $h$ but depending on the polynomial degree $k$.

The following property is necessary to obtain optimal estimates of the convergence rate.

Definition 2.6 (optimal polynomial approximation). We say that the mesh sequence $\mathcal{T}_{\mathcal{H}}$ has optimal polynomial approximation properties if for all $h \in \mathcal{H}$, all $T \in \mathcal{T}_{h}$, and all polynomial degree $k \geq 0$, there is a polynomial $p^{k} \in \mathbb{P}_{d}^{k}(T)$ such that, for all $s \in\{0, \ldots, k+1\}$ and all $v \in H^{s}(T)$, there holds

$$
\left|v-p^{k}\right|_{H^{m}(T)} \leq C_{\mathrm{app}} h_{T}^{s-m}|v|_{H^{s}(T)} \quad \forall m \in\{0, \ldots, s\}
$$

with $C_{\text {app }}$ independent of both $T$ and $h$.

The approximation property is a consequence of the Bramble-Hilbert lemma (see, e.g., [10], Lem. 4.3.8) for mesh sequences featuring general elements whose chunkiness parameter is bounded from above uniformly with respect to $h$. We recall that the chunkiness parameter is defined for an element $T \in \mathcal{T}_{h}, h \in \mathcal{H}$, as the ratio $h_{T} / r_{\max , T}$, where $r_{\max , T}:=\sup \left\{r \in \mathbb{R}^{+} \mid T\right.$ is star-shaped with respect to a ball of radius $\left.r\right\}$. Using the optimality of the $L^{2}$-orthogonal projection in the $L^{2}$-norm, one can easily infer that the $L^{2}$-orthogonal projection has optimal polynomial approximation properties on mesh sequences with optimal approximation properties; see [24], Lemma 1.58. In what follows, we mainly use the optimal polynomial approximation for polynomial degrees up to 1 . Higher polynomial degrees are only needed in view of $k$-adaptivity; $c f$. Remark 2.13.

In order to obtain a piecewise affine representation, we associate the unique degree of freedom of each element to a point inside the cell.

Definition 2.7 (cell centers). Let $\mathcal{T}_{\mathcal{H}}$ be a shape- and contact-regular mesh sequence. We say that $\mathcal{T}_{\mathcal{H}}$ admits a set of cell centers if (i) for every $T \in \mathcal{T}_{h}$ there exists a point $\mathbf{x}_{T}$ such that $T$ is star-shaped with respect to $\mathbf{x}_{T}$ (the cell-center) and (ii) there exists $\varrho_{3}>0$ such that for all $h \in \mathcal{H}$, all $T \in \mathcal{T}_{h}$, and all $F \in \mathcal{F}_{T}$,

$$
d_{T, F}:=\operatorname{dist}\left(\mathbf{x}_{T}, F\right) \geq \varrho_{3} h_{T}
$$

Definition 2.8 (admissible mesh sequence). We say that the mesh sequence $\mathcal{T}_{\mathcal{H}}$ is admissible if it is shape- and contact-regular, it possesses optimal approximation properties, and it admits a set of cell centers.

In what follows we always work with admissible mesh sequences. For the sake of brevity, this assumption is understood in most of the cases. Admissible mesh sequences include general polyhedral discretizations with possibly nonconforming interfaces; see Figure 1 for an example. For all $h \in \mathcal{H}$ we can define a pyramidal submesh of $\mathcal{T}_{h}$ as follows:

$$
\mathcal{S}_{h}:=\left\{\mathcal{P}_{T, F}\right\}_{T \in \mathcal{T}_{h}, F \in \mathcal{F}_{T}},
$$

where, for all $T \in \mathcal{T}_{h}$ and all $F \in \mathcal{F}_{T}, \mathcal{P}_{T, F}$ denotes the open pyramid of apex $\mathbf{x}_{T}$ and base $F$, i.e.,

$$
\mathcal{P}_{T, F}:=\left\{\mathbf{x} \in T|\exists \mathbf{y} \in F \backslash \partial F, \exists \theta \in(0,1)| \mathbf{x}=\theta \mathbf{y}+(1-\theta) \mathbf{x}_{T}\right\}
$$

The pyramids $\left\{\mathcal{P}_{T, F}\right\}_{T \in \mathcal{T}_{h}, F \in \mathcal{F}_{T}}$ are non-degenerated owing to assumption (2.6). Since faces are planar, for all $T \in \mathcal{T}_{h}$ and all $F \in \mathcal{F}_{T}$ there holds

$$
\left|\mathcal{P}_{T, F}\right|_{d}=\frac{|F|_{d-1} d_{T, F}}{d}
$$



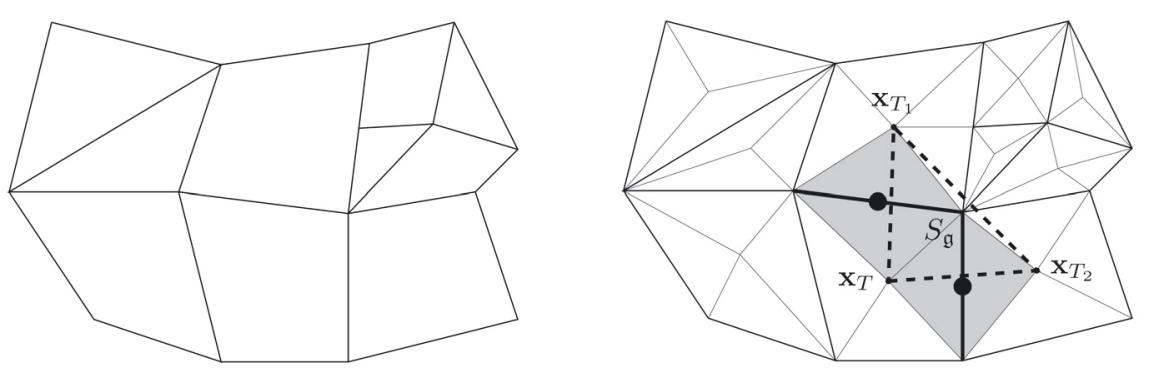

Figure 1. Mesh $\mathcal{T}_{h}$ (left panel), submesh $\mathcal{S}_{h}$ (right panel, thin lines), and simplex $S_{\mathfrak{g}}$ (right panel, dashed lines) for the L-group $\mathfrak{g}$ in bold line. The faces belonging to the group $\mathfrak{g}$ are marked with a dot; the patch $\mathcal{P}_{\mathfrak{g}}$ is shaded.

and, for all $T \in \mathcal{T}_{h}$,

$$
\sum_{F \in \mathcal{F}_{T}}\left|\mathcal{P}_{T, F}\right|_{d}=\sum_{F \in \mathcal{F}_{T}} \frac{|F|_{d-1} d_{T, F}}{d}=|T|_{d}
$$

\subsection{The L-construction}

In this section we briefly recall the L-construction originally introduced in [3] and analyzed in [4]; see also Cao et al. [17]. This construction is an important ingredient in the definition of the ccG spaces presented in this work. Let $P_{\Omega}$ denote a given partition of $\Omega$ into bounded connected polyhedral domains corresponding, e.g., to regions with different physical properties. When considering refined mesh sequences $\mathcal{T}_{\mathcal{H}}$, throughout the rest of this work we understand the following assumption, which is stated once and for all for the sake of conciseness.

Assumption 2.9 (mesh compatibility). For all $h \in \mathcal{H}, \mathcal{T}_{h}$ is compatible with the partition $P_{\Omega}$.

Let $\boldsymbol{\kappa} \in\left[L^{\infty}(\Omega)\right]^{d, d}$ denote a symmetric, uniformly elliptic tensor field piecewise constant on $P_{\Omega}$. Clearly, owing to Assuption 2.9, $\boldsymbol{\kappa}$ is such that

$$
\forall h \in \mathcal{H}, \quad \kappa \in\left[\mathbb{P}_{d}^{0}\left(\mathcal{T}_{h}\right)\right]^{d, d} .
$$

We assume that the spectrum of $\boldsymbol{\kappa}(\mathbf{x})$ is bounded from below by $\underline{\lambda}$ for a.e. $\mathbf{x} \in \Omega$ and we let $\bar{\lambda}:=\|\boldsymbol{\kappa}\|_{\left[L^{\infty}(\Omega)\right]^{d, d}}$. We also denote by $\boldsymbol{\kappa}^{1 / 2}$ the piecewise symmetric, uniformly elliptic tensor field such that $\boldsymbol{\kappa}^{1 / 2} \boldsymbol{\kappa}^{1 / 2}=\boldsymbol{\kappa}$ in $\Omega$. For all $F \in \mathcal{F}_{h}$ and all $T \in \mathcal{T}_{F}$ we denote the diffusion coefficient in the normal direction by

$$
\lambda_{T, F}:=\boldsymbol{\kappa}_{\mid T} \mathbf{n}_{F} \cdot \mathbf{n}_{F} .
$$

The key idea of the L-construction is to use $d$ cell and boundary face values (provided, in this case, by a homogeneous boundary condition) to express a continuous piecewise affine function with continuous diffusive fluxes. The values are selected using $d$ neighboring faces belonging to a cell and sharing a common vertex. More precisely, we define the set of L-groups as follows:

$$
\mathcal{G}:=\left\{\mathfrak{g} \subset \mathcal{F}_{T} \cap \mathcal{F}_{P}, T \in \mathcal{T}_{P}, P \in \mathcal{N}_{h} \mid \operatorname{card}(\mathfrak{g})=d\right\},
$$

with $\mathcal{F}_{T}$ given by $(2.1)$ and $\mathcal{F}_{P}$ and $\mathcal{T}_{P}$ given by (2.2). For each $\mathfrak{g} \in \mathcal{G}$ we select a primary element $T_{\mathfrak{g}}$ such that $\mathfrak{g} \subset \mathcal{F}_{T_{\mathfrak{g}}}$; see Figure 2a. Such an element may not be unique as non-convex elements are allowed. We let, for the sake of brevity,

$$
\mathfrak{g}^{\mathrm{i}}:=\mathfrak{g} \cap \mathcal{F}_{h}^{\mathrm{i}}, \quad \mathfrak{g}^{\mathrm{b}}:=\mathfrak{g} \cap \mathcal{F}_{h}^{\mathrm{b}} .
$$



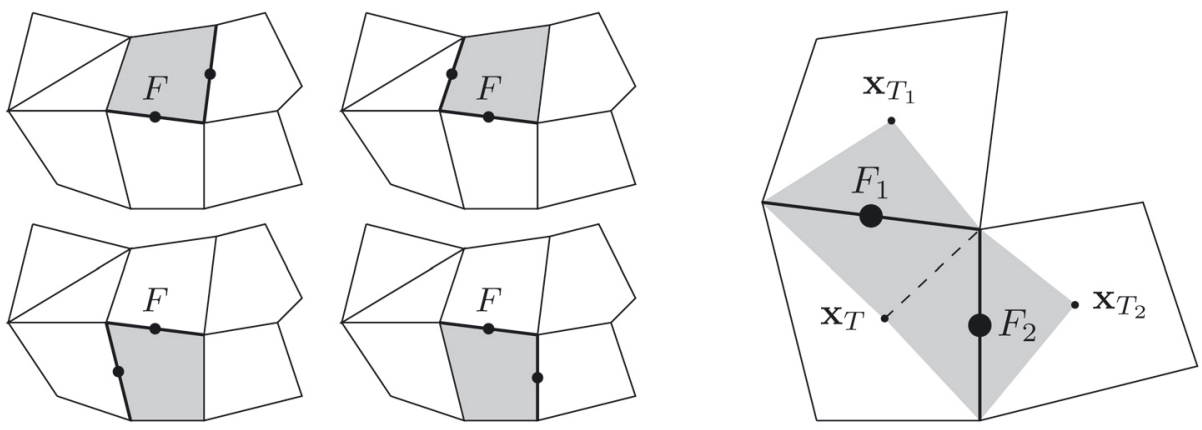

(a) L-groups containing the face $F$. The primary element $T_{\mathfrak{g}}$ such that $\mathfrak{g} \subset \mathcal{F}_{T}$ is shaded

(b) L-construction

Figure 2. L-construction. Group faces are marked with a dot.

It is also useful to introduce a symbol for the set of cells concurring in the L-construction as well as for the union of the pyramids based on the group faces (see Fig. 2): For all $\mathfrak{g} \in \mathcal{G}$,

$$
\mathcal{T}_{\mathfrak{g}}:=\left\{T \in \mathcal{T}_{h} \mid T \in \mathcal{T}_{F}, F \in \mathfrak{g}\right\}, \quad \mathcal{P}_{\mathfrak{g}}:=\bigcup_{F \in \mathfrak{g}, T \in \mathcal{T}_{F}} \mathcal{P}_{T, F} .
$$

The patch $\mathcal{P}_{\mathfrak{g}}$ is shaded in the right panel of Figure 1 and in Figure $2 \mathrm{~b}$. Let now $\mathfrak{g} \in \mathcal{G}$. In what follows, for any set $D \subset \mathbb{R}^{d}$ of codimension $l$ we denote by $\langle\varphi\rangle_{D}$ the average $\langle\varphi\rangle_{D}:=\int_{D} \varphi /|D|_{d-l}$. For all $\mathbf{v}_{h} \in \mathbb{V}_{h}$ we construct the piecewise function $\xi_{\mathbf{v}_{h}}^{\mathfrak{g}}$ affine inside every pyramid in the the family $\left\{\mathcal{P}_{T, F}\right\}_{F \in \mathfrak{g}, T \in \mathcal{T}_{F}}$ and such that:

(i) $\xi_{\mathbf{v}_{h}}^{\mathfrak{g}}\left(\mathbf{x}_{T}\right)=v_{T}$ for all $T \in \mathcal{T}_{\mathfrak{g}}$ and $\left\langle\xi_{\mathbf{v}_{h}}^{\mathfrak{g}}\right\rangle_{F}=\xi_{\mathbf{v}_{h}}^{\mathfrak{g}}\left(\overline{\mathbf{x}}_{F}\right)=0$ for all $F \in \mathfrak{g}^{\mathrm{b}}$;

(ii) $\xi_{\mathbf{v}_{h}}^{\mathfrak{h}}$ is affine inside the primary element $T_{\mathfrak{g}}$ and it is continuous across every interface in the group: for all $F \in \mathfrak{g}^{\mathrm{i}}$ such that $F \subset \partial T_{1} \cap \partial T_{2}$,

$$
\forall \mathbf{x} \in F, \quad \xi_{\mathbf{v}_{h} \mid T_{1}}^{\mathfrak{g}}(\mathbf{x})=\xi_{\mathbf{v}_{h} \mid T_{2}}^{\mathfrak{g}}(\mathbf{x})
$$

(iii) $\xi_{\mathbf{v}_{h}}^{\mathfrak{g}}$ has continuous diffusive flux across every interface in the group: For all $F \in \mathfrak{g}^{\mathrm{i}}$ such that $F \subset \partial T_{1} \cap \partial T_{2}$,

$$
\left(\kappa \nabla \xi_{\mathbf{v}_{h}}^{\mathfrak{g}}\right)_{\mid T_{1}} \cdot \mathbf{n}_{F}=\left(\kappa \nabla \xi_{\mathbf{v}_{h}}^{\mathfrak{g}}\right)_{\mid T_{2}} \cdot \mathbf{n}_{F}
$$

(The quantities in both sides are constant since $\xi_{\mathbf{v} h}^{\mathfrak{g}}$ is piecewise affine and the face $F$ is planar.) The following result is instrumental to derive approximation properties for the ccG space.

Proposition 2.10 (L-construction). For all $\mathbf{v}_{h} \in \mathbb{V}_{h}$ and all $\mathfrak{g} \in \mathcal{G}$, there holds

$$
\mathbf{A}_{\mathfrak{g}}\left(\nabla \xi_{\mathbf{v}_{h}}^{\mathfrak{g}}\right)_{\mid T_{\mathfrak{g}}}=\mathbf{b}_{\mathfrak{g}}\left(\mathbf{v}_{h}\right)
$$

where the matrix $\mathbf{A}_{\mathfrak{g}} \in \mathbb{R}^{d, d}$ and the linear application $\mathbf{b}_{\mathfrak{g}}: \mathbb{V}_{h} \rightarrow \mathbb{R}^{d}$ are defined row-wise by

$$
\mathbf{A}_{\mathfrak{g}}:=\left[\begin{array}{c}
\left(\frac{\lambda_{T, F}}{d_{T, F}}\left(\mathbf{x}_{T}-\mathbf{x}_{T_{\mathfrak{g}}}\right)+\boldsymbol{\kappa}_{T_{\mathfrak{g}}} \mathbf{n}_{T_{\mathfrak{g}}, F}+\boldsymbol{\kappa}_{T, F} \mathbf{n}_{T, F}\right)_{\mathfrak{g}^{\mathrm{i}} \ni F \subset T_{\mathfrak{g}} \cap T}^{t} \\
\left(\frac{\lambda_{T_{\mathfrak{g}}, F}}{d_{T_{\mathfrak{g}}, F}}\left(\overline{\mathbf{x}}_{F}-\mathbf{x}_{T_{\mathfrak{g}}}\right)\right)_{F \in \mathfrak{g}^{\mathrm{b}}}^{t}
\end{array}\right], \quad \mathbf{b}_{\mathfrak{g}}\left(\mathbf{v}_{h}\right)=\left[\begin{array}{c}
\left(\frac{\lambda_{T, F}}{d_{T, F}}\left(v_{T}-v_{T_{\mathfrak{g}}}\right)\right)_{\mathfrak{g}^{\mathrm{i}} \ni F \subset T_{\mathfrak{g}} \cap T} \\
\left(-\frac{\lambda_{T_{\mathfrak{g}}, F}}{d_{T_{\mathfrak{g}}, F}} v_{T_{\mathfrak{g}}}\right)_{F \in \mathfrak{g}^{\mathrm{b}}}
\end{array}\right] .
$$

Proof. See [4], Lemma 3.1. 
In order to express $\xi_{\mathbf{v}_{h}}^{\mathfrak{g}}$ terms of the values $\left\{v_{T}\right\}_{T \in T_{\mathfrak{g}}}$, the matrix $\mathbf{A}_{\mathfrak{g}}$ must be invertible. Simple sufficient inversibility conditions are discussed in what follows. In practice, however, the inversibility can be checked directly, and backup strategies can be devised; see Remark 2.12.

\subsection{The ccG space}

In this section we introduce a ccG space based on the L-construction and on a local gradient inspired by Green's formula. For a face $F \in \mathcal{F}_{h}^{\mathrm{i}}$, let $\mathcal{G}_{F}$ denote the set of L-groups containing $F$,

$$
\mathcal{G}_{F}:=\{\mathfrak{g} \in \mathcal{G} \mid F \in \mathfrak{g}\} .
$$

Assumption 2.11 (existence of an L-group leading to an invertible matrix for each interface). We assume that, for all $F \in \mathcal{F}_{h}^{\mathrm{i}}, \mathcal{G}_{F}$ is non-empty and there exists $\mathfrak{g} \in \mathcal{G}_{F}$ such that the matrix $\mathbf{A}_{\mathfrak{g}}$ defined by (2.11) is invertible.

For the sake of simplicity, Assumption 2.11 holds tacitly from this point on. Should this not be true, backup strategies can be envisaged, as discussed in Remark 2.12. The discrete gradient is obtained as follows:

(i) For every $F \in \mathcal{F}_{h}^{\mathrm{i}}$ we select a unique L-group $\mathfrak{g}_{F}$ yielding an invertible matrix and, for all $\mathbf{v}_{h} \in \mathbb{V}_{h}$, we denote by $\xi_{\mathbf{v}_{h}}^{\mathfrak{g}_{F}}$ the piecewise affine function obtained from the L-construction and supported in the patch $\mathcal{P}_{\mathfrak{g}_{F}}$. Whenever more than one such group is present, we select $\mathfrak{g} \in \mathcal{G}_{F}$ for which $\left\|\mathbf{A}_{\mathfrak{g}}^{-1}\right\|_{2}$ is minimal (this ensures the best approximation properties; see Lem. 3.6 and Assumption 3.7). For convenience of notation, for all boundary faces $F \subset \partial T \cap \partial \Omega$ we introduce the affine function $\xi_{\mathbf{v}_{h}}$ on $\mathcal{P}_{T, F}$ such that $\xi_{\mathbf{v}_{h} \mid F}^{\mathfrak{g}_{F}} \equiv 0$ on $F$ and $\xi_{\mathbf{v}_{h}}^{\mathfrak{g}_{F}}\left(\mathbf{x}_{T}\right)=v_{T}$. Such a function is well-defined since faces are planar and cell centers cannot lie on cell boundaries.

(ii) We then define the trace reconstruction operator $\mathfrak{T}_{h}: \mathbb{V}_{h} \rightarrow \mathbb{R}^{\mathcal{F}_{h}}$ which realizes the mapping $\mathbb{V}_{h} \ni \mathbf{v}_{h} \mapsto$ $\mathfrak{T}_{h}\left(\mathbf{v}_{h}\right)=\left(v_{F}\right)_{F \in \mathcal{F}_{h}}$ with $\left(v_{F}\right)_{F \in \mathcal{F}_{h}} \in \mathbb{R}^{\mathcal{F}_{h}}$ and, for all $F \in \mathcal{F}_{h}$,

$$
v_{F}=\left\langle\xi_{\mathbf{v}_{h}}^{\mathfrak{g}_{F}}\right\rangle_{F}=\xi_{\mathbf{v}_{h}}^{\mathfrak{g}_{F}}\left(\overline{\mathbf{x}}_{F}\right) .
$$

(iii) The gradient reconstruction operator $\mathfrak{G}_{h}: \mathbb{V}_{h} \rightarrow\left[\mathbb{P}_{d}^{0}\left(\mathcal{T}_{h}\right)\right]^{d}$ is defined following Droniou and Eymard [26] as the application $\mathbb{V}_{h} \ni \mathbf{v}_{h} \mapsto \mathfrak{G}_{h}\left(\mathbf{v}_{h}\right) \in\left[\mathbb{P}_{d}^{0}\left(\mathcal{T}_{h}\right)\right]^{d}$ with

$$
\forall T \in \mathcal{T}_{h}, \quad \mathfrak{G}_{h}\left(\mathbf{v}_{h}\right)_{\mid T}=\frac{1}{|T|_{d}} \sum_{F \in \mathcal{F}_{T}}|F|_{d-1}\left(v_{F}-v_{T}\right) \mathbf{n}_{T, F}
$$

where we have set $\left(v_{F}\right)_{F \in \mathcal{F}_{h}}=\mathfrak{T}_{h}\left(\mathbf{v}_{h}\right)$.

The expression (2.13) is inspired by Green's formula. As such, $v_{F}$ has to be related to the average on the face $F$. For affine functions, the average coincides with the barycentric value, thereby justifying the choice in (2.12). The linear reconstruction operator $\mathfrak{R}_{h}^{\mathrm{ccg}}: \mathbb{V}_{h} \rightarrow \mathbb{P}_{d}^{1}\left(\mathcal{T}_{h}\right)$ leading to the ccG space is finally defined as the mapping $\mathbb{V}_{h} \ni \mathbf{v}_{h} \mapsto v_{h} \in \mathbb{P}_{d}^{1}\left(\mathcal{T}_{h}\right)$ with

$$
\forall T \in \mathcal{T}_{h}, \forall \mathbf{x} \in T, \quad v_{h \mid T}(\mathbf{x})=v_{T}+\mathfrak{G}_{h}\left(\mathbf{v}_{h}\right)_{\mid T} \cdot\left(\mathbf{x}-\mathbf{x}_{T}\right)
$$

In the above formula, $v_{T}$ is interpreted as the point value in $\mathbf{x}_{T}$. The incomplete polynomial space $V_{h}^{\mathrm{ccg}}$ upon which the ccG method relies is the image of the vector space of degrees of freedom $\mathbb{V}_{h}$ through $\mathfrak{R}_{h}^{\mathrm{ccg}}$,

$$
V_{h}^{\mathrm{ccg}}:=\mathfrak{R}_{h}^{\mathrm{ccg}}\left(\mathbb{V}_{h}\right) \subset \mathbb{P}_{d}^{1}\left(\mathcal{T}_{h}\right) .
$$

Proceeding by contradiction, it is a simple matter to prove that $\mathfrak{R}_{h}^{\text {ccg }}$ is injective from $\mathbb{V}_{h}$ to $\mathbb{P}_{d}^{1}\left(\mathcal{T}_{h}\right)$ and, hence, bijective from $\mathbb{V}_{h}$ to $V_{h}^{\mathrm{ccg}}$. It is also useful to observe that the discrete space $V_{h}^{\mathrm{ccg}}$ is contained in the larger broken Sobolev space $H^{1}\left(\mathcal{T}_{h}\right)$. 
Remark 2.12 (backup strategies). Numerical evidence [3,4] shows that Assumption 2.11 is true in most circumstances. In the presence of highly heterogeneous diffusion tensors or for extremely deformed meshes, it may occur, however, that no L-group yielding an invertible matrix can be found for some interfaces. In this case, several backup strategies are possible, and the choice can be mostly guided by the implementation at hand. We mention two possibilities, although others are possible. A first strategy relying on the tight link of ccG methods to dG methods consists in using a full $\mathbb{P}_{d}^{1}$ basis on the mesh elements which have at least one face for which no invertible L-group exists. This results in a local increase of the number of unknowns but yields a completely robust method. Whenever exactly one unknown per cell is allowed, the L-construction can be replaced by the barycentric interpolator of [33], Section 2.2. The method can then be constructed provided the $d$ points involved in the barycentric interpolation form a non-degenerate simplex (an extremely mild mesh regularity assumption). In this case, however, a local loss of precision may be observed as the barycentric interpolator does not honor the heterogeneity of $\boldsymbol{\kappa}$. We emphasize that no backup strategy whatsoever was required in the numerical examples of Section 5.

Remark 2.13 ( $k$-adaptivity). In the spirit of the previous remark, the polynomial degree can be adapted in ccG methods by using full polynomial spaces inside selected elements. This is naturally handled whenever the discrete formulation relying on the ccG space is inspired by a dG method, as is the case for all the examples provided in this work.

\subsection{Discrete functional analysis}

This section collects some discrete functional analysis results that are used in the rest of the paper. The material is mainly adapted from [22], Section 6 , to which we refer for further details. We state, in particular, the Sobolev embeddings for broken polynomial spaces in the Hilbertian case and the discrete counterpart of the Rellich-Kondrachov theorem. To this end, we introduce the following norm on $H^{1}\left(\mathcal{T}_{h}\right)$ :

$$
\|v\|^{2}:=\left\|\nabla_{h} v\right\|_{\left[L^{2}(\Omega)\right]^{d}}^{2}+|v|_{\mathrm{J}}^{2}, \quad|v|_{J}^{2}:=\sum_{F \in \mathcal{F}_{h}} \frac{1}{h_{F}}\|\llbracket v \rrbracket\|_{L^{2}(F)}^{2}
$$

where $\nabla_{h}$ denotes the broken gradient on $\mathcal{T}_{h}$, i.e., for all $v \in H^{1}\left(\mathcal{T}_{h}\right), \nabla_{h} v_{\mid T}=\nabla\left(v_{\mid T}\right)$ for all $T \in \mathcal{T}_{h}$.

Theorem 2.14 (discrete Sobolev embeddings, Hilbertian case). For all $q$ such that (i) $1 \leq q<+\infty$ if $d \leq 2$, (ii) $1 \leq q \leq \frac{2 d}{d-2}$ if $d>2$, there is $\sigma_{q}$ independent of $h$ such that

$$
\forall v_{h} \in \mathbb{P}_{d}^{k}\left(\mathcal{T}_{h}\right), \quad\left\|v_{h}\right\|_{L^{q}(\Omega)} \leq \sigma_{q}\left\|v_{h}\right\| .
$$

The constant $\sigma_{q}$ additionally depends on $k,|\Omega|_{d}$ and on the mesh regularity parameters.

The discrete Poincaré inequality is obtained as a special case for $q=2$. For a proof in the more general non-Hilbertian case we refer to [22]. Sequences in

$$
V_{\mathcal{H}}^{\mathrm{ccg}}:=\left(V_{h}^{\mathrm{ccg}}\right)_{h \in \mathcal{H}}
$$

uniformly bounded in the ||$|\|| \mid$-norm possess an important compactness property which we discuss in what follows. Following the idea of Brezzi et al. [13] modified according to [5], Section 3.1, we introduce for all $F \in \mathcal{F}_{h}$ the local lifting operator $\mathbf{r}_{\omega, F}: L^{2}(F) \rightarrow\left[\mathbb{P}_{d}^{0}\left(\mathcal{T}_{h}\right)\right]^{d}$ which maps every $\varphi \in L^{2}(F)$ onto $\mathbf{r}_{\omega, F}(\varphi) \in\left[\mathbb{P}_{d}^{0}\left(\mathcal{T}_{h}\right)\right]^{d}$ solution to:

$$
\int_{\Omega} \mathbf{r}_{\omega, F}(\varphi) \cdot \tau_{h}=\int_{F} \varphi\left\{\tau_{h}\right\}_{\omega} \cdot \mathbf{n}_{F}, \quad \forall \tau_{h} \in\left[\mathbb{P}_{d}^{0}\left(\mathcal{T}_{h}\right)\right]^{d} .
$$

An important remark is that, for a given face $F \in \mathcal{F}_{h}$, the lifting operator $\mathbf{r}_{\omega, F}$ is supported in $\bigcup_{T \in \mathcal{T}_{F}} T$. For further use we also introduce the global lifting

$$
\mathbf{R}_{\omega, h}(\varphi):=\sum_{F \in \mathcal{F}_{h}} \mathbf{r}_{\omega, F}(\varphi)
$$


If $\omega_{T_{1}, F}=\omega_{T_{2}, F}=1 / 2$ for all $F \in \mathcal{F}_{h}^{\mathrm{i}}$ with $F \subset \partial T_{1} \cap \partial T_{2}$ we simply write $\mathbf{r}_{F}$ and $\mathbf{R}_{h}$. The lifting operators can be used to define a corrected discrete gradient accounting for the jumps across mesh interfaces and on $\partial \Omega$. More precisely, we introduce the linear operator $\mathbf{G}_{\omega, h}: H^{1}\left(\mathcal{T}_{h}\right) \rightarrow\left[L^{2}(\Omega)\right]^{d}$ defined as follows: For all $v \in H^{1}\left(\mathcal{T}_{h}\right)$,

$$
\mathbf{G}_{\omega, h}(v):=\nabla_{h} v-\mathbf{R}_{\omega, h}(\llbracket v \rrbracket) .
$$

As before, if $\omega_{T_{1}, F}=\omega_{T_{2}, F}=1 / 2$ for all $F \in \mathcal{F}_{h}^{\mathrm{i}}$ with $F \subset \partial T_{1} \cap \partial T_{2}$ we omit the subscript $\omega$ and write $\mathbf{G}_{h}$.

Theorem 2.15 (discrete Rellich-Kondrachov). Let $\left(v_{h}\right)_{h \in \mathcal{H}}$ be a sequence in $\left(\mathbb{P}_{d}^{k}\left(\mathcal{T}_{h}\right)\right)_{h \in \mathcal{H}}, k \geq 0$, uniformly bounded in the $\|\cdot\| \|-n o r m$. Then, there exists a function $v \in H_{0}^{1}(\Omega)$ such that as $h \rightarrow 0$, up to a subsequence $v_{h} \rightarrow v$ strongly in $L^{2}(\Omega)$.

Proof. See [22], Section 6.

Lemma 2.16 (weak asymptotic consistency of $\mathbf{G}_{\omega, h}$ for sequences of discrete functions). Let $\left(v_{h}\right)_{h \in \mathcal{H}}$ be a sequence in $\left(\mathbb{P}_{d}^{k}\left(\mathcal{T}_{h}\right)\right)_{h \in \mathcal{H}}, k \geq 0$, uniformly bounded in the $\left\|\left|\|\mid\|\right.\right.$-norm. Then, as $h \rightarrow 0, \mathbf{G}_{\omega, h}\left(v_{h}\right) \rightarrow \nabla v$ weakly in $\left[L^{2}(\Omega)\right]^{d}$, where $v \in H_{0}^{1}$ is the limit provided by Theorem 2.15 .

Proof. Denote by $\pi_{h}^{1}$ the $L^{2}$-orthogonal projection onto $\left[\mathbb{P}_{d}^{1}\left(\mathcal{T}_{h}\right)\right]^{d}$. To prove the weak convergence of $\mathbf{G}_{\omega, h}\left(v_{h}\right)$ to $\nabla v$, let $\Phi \in\left[C_{0}^{\infty}(\Omega)\right]^{d}$, set $\Phi_{h}:=\pi_{h}^{1} \Phi$, and observe that

$$
\int_{\Omega} \mathbf{G}_{\omega, h}\left(v_{h}\right) \cdot \Phi=-\int_{\Omega} v_{h} \nabla \cdot \Phi+\sum_{T \in \mathcal{T}_{h}} \int_{\partial T} \Phi \cdot \mathbf{n}_{T} v_{h}-\sum_{F \in \mathcal{F}_{h}} \int_{\Omega} \mathbf{r}_{\omega, F}\left(\llbracket v_{h} \rrbracket\right) \cdot \Phi_{h}=-\int_{\Omega} v_{h} \nabla \cdot \Phi+\sum_{F \in \mathcal{F}_{h}} \int_{F} \llbracket v_{h} \rrbracket\left\{\Phi-\Phi_{h}\right\}_{\omega} \cdot \mathbf{n}_{F},
$$

where we have used the definition of the $L^{2}$-orthogonal projection, the fact that $\{\Phi\}_{\omega}=\Phi$ on every $F \in \mathcal{F}_{h}$, and (2.16). Denote by $\mathfrak{T}_{1}$ and $\mathfrak{T}_{2}$ the addends in the right-hand side. Clearly, $\mathfrak{T}_{1} \rightarrow \int_{\Omega} v \nabla \cdot \Phi$. For the second term, the Cauchy-Schwarz inequality yields $\mathfrak{T}_{2} \leq\left|v_{h}\right|_{J} \times\left(\sum_{F \in \mathcal{F}_{h}} h_{F} \int_{F}\left|\left\{\Phi-\Phi_{h}\right\}_{\omega}\right|^{2}\right)^{1 / 2}$, which tends to zero owing to the approximation properties of the $L^{2}$-orthogonal projection for the smooth function $\Phi$ together with the trace inequality (2.5) and the fact that $\left|v_{h}\right|_{\mathrm{J}}$ is uniformly bounded by assumption.

It is important to observe that, since $V_{h}^{\text {ccg }} \subset \mathbb{P}_{d}^{1}\left(\mathcal{T}_{h}\right)$, both Theorems 2.14 and 2.15 and Lemma 2.16 hold $a$ fortiori for sequences of functions in the ccG spaces $V_{\mathcal{H}}^{\text {ccg }}$.

\section{PuRE DifFusion}

\subsection{The discrete problem}

The space $V_{h}^{\text {ccg }}$ is used in this section to discretize the heterogeneous diffusion problem

$$
\begin{aligned}
& -\nabla \cdot(\kappa \nabla u)=f \quad \text { in } \Omega \text {, } \\
& u=0 \quad \text { on } \partial \Omega \text {, }
\end{aligned}
$$

with source term $f \in L^{2}(\Omega)$. The weak form of this problem is the following:

$$
\text { Find } u \in V \text { s.t. } a(u, v)=\int_{\Omega} f v \text { for all } v \in V \text {, }
$$

with $V=H_{0}^{1}(\Omega)$ and $a(u, v):=\int_{\Omega} \boldsymbol{\kappa} \nabla u \cdot \nabla v$. The functions in $V_{h}^{\text {ccg }}$ are possibly discontinuous across interfaces, and $V_{h}^{\text {ccg }}$ is therefore not $V$-conforming. In order to devise a suitable discretization, we take inspiration from the work of Arnold [6] on the weak enforcement of potential continuity across interfaces. In particular, we consider the modification proposed by Di Pietro et al. [25] in the context of degenerate diffusion-advectionreaction problems to attain robustness with respect to the diffusion tensor $\boldsymbol{\kappa}$. This requires to relate the weights 
introduced in Definition 2.1 to the diffusion coefficient on both sides of an interface. The idea of diffusiondependent weights can be traced back to the work of Burman and Zunino [16] on mortaring techniques for a singularly perturbed diffusion-advection equation. For all $F \in \mathcal{F}_{h}^{\mathrm{i}}$ such that $F \subset \partial T_{1} \cap \partial T_{2}$, we let

$$
\omega_{T_{1}, F}:=\frac{\lambda_{T_{2}, F}}{\lambda_{T_{1}, F}+\lambda_{T_{2}, F}}, \quad \omega_{T_{2}, F}:=\frac{\lambda_{T_{1}, F}}{\lambda_{T_{1}, F}+\lambda_{T_{2}, F}} .
$$

The analysis in the spirit of Céa's Lemma requires to extend the discrete bilinear form to a continuous space containing the exact solution. In order to guarantee that boundary terms remain well-defined when doing so, it is useful to introduce a space $V_{*}$ featuring additional local regularity with respect to $V$.

Definition 3.1 ( $\operatorname{space} V_{*}$ ). We let $V_{*}$ denote the subspace of $V$ such that

$$
V_{*}:=V \cap H^{2}\left(P_{\Omega}\right),
$$

with $H^{2}\left(P_{\Omega}\right)$ spanned by the functions whose restriction to every $S \in P_{\Omega}$ (with $P_{\Omega}$ defined in Sect. 2.2) is in $H^{2}(S)$.

Remark 3.2 ( $L^{2}$-regularity of traces). Using classical trace inequalities (see, e.g., Brenner and Scott [10], Chap. 1) it is inferred that there exists $C$ such that, for all $v \in V_{*}$ and all $T \in \mathcal{T}_{h}$,

$$
\|\nabla v\|_{\left[L^{2}(\partial T)\right]^{d}} \leq C\|\nabla v\|_{\left[L^{2}(T)\right]^{d}}^{1 / 2}\|\nabla v\|_{\left[H^{1}(T)\right]^{d, d}}^{1 / 2},
$$

that is to say, since $v \in H^{2}(T)$, the gradient of $v$ has square-integrable trace on $\partial T$ for all $T \in \mathcal{T}_{h}$. This implies, in particular, that both $\nabla v_{\mid T} \cdot \mathbf{n}_{T}$ and, using $(2.9),\left(\boldsymbol{\kappa}^{1 / 2} \nabla v\right)_{\mid T} \cdot \mathbf{n}_{T}$ have square-integrable traces on $\partial T$.

The space which is assumed to contain both the discrete and the continuous solution is defined as

$$
V_{* h}:=V_{h}^{\mathrm{ccg}}+V_{*} .
$$

The additional regularity assumption $u \in V_{*}$ is made in what follows to simplify the exposition and write integrals instead of duality pairings. For some details on how to relax this assumption we refer to Di Pietro and Ern [23] and references therein. Regularity results for the solution of problem (3.1) can be found, e.g., in the work of Kellogg [38]; see also Nicaise and Sändig [40].

We are now ready to define the bilinear form $a_{h} \in \mathcal{L}\left(V_{* h} \times V_{* h}, \mathbb{R}\right)$,

$$
a_{h}(v, w):=\int_{\Omega} \boldsymbol{\kappa} \nabla_{h} v \cdot \nabla_{h} w-\sum_{F \in \mathcal{F}_{h}} \int_{F}\left\{\boldsymbol{\kappa} \nabla_{h} v\right\}_{\omega} \cdot \mathbf{n}_{F} \llbracket w \rrbracket-\sum_{F \in \mathcal{F}_{h}} \int_{F} \llbracket v \rrbracket\left\{\boldsymbol{\kappa} \nabla_{h} w\right\}_{\omega} \cdot \mathbf{n}_{F}+\sum_{F \in \mathcal{F}_{h}} \eta \frac{\gamma_{F}}{h_{F}} \int_{F} \llbracket v \rrbracket \llbracket w \rrbracket,
$$

where $\eta>0$ denotes a user-dependent penalty parameter while $\gamma_{F}$ is such that

$$
\gamma_{F}:= \begin{cases}2 \frac{\lambda_{T_{1}, F} \lambda_{T_{2}, F}}{\lambda_{T_{1}, F}+\lambda_{T_{2}, F}} & \text { if } F \in \mathcal{F}_{h}^{\mathrm{i}}, F \subset \partial T_{1} \cap \partial T_{2}, \\ \lambda_{T, F} & \text { if } F \in \mathcal{F}_{h}^{\mathrm{b}}, F \subset \partial T \cap \partial \Omega .\end{cases}
$$

The penalty parameter $\gamma_{F}$, molded after [25], contains the harmonic averaging of the normal diffusion coefficient on both sides of an interface. This, together with the definition of the weights (3.3), ensures that the correct amount of penalization is added to control the second and third term in the right hand side of (3.4) when proving the coercivity of $a_{h}$ with respect to the natural energy norm defined by (3.7). As a result, the coercivity constant does not depend on $\boldsymbol{\kappa} ; c f$. Lemma 3.3. The discrete problem reads

$$
\text { Find } u_{h} \in V_{h}^{\mathrm{ccg}} \text { s.t. } a_{h}\left(u_{h}, v_{h}\right)=\int_{\Omega} f v_{h} \text { for all } v_{h} \in V_{h}^{\mathrm{ccg}} \text {. }
$$




\subsection{Basic error estimate}

We introduce the following data dependent norms on $V_{* h}$ :

$$
\left\|v \left|\left\|_{\boldsymbol{\kappa}}^{2}:=\right\| \boldsymbol{\kappa}^{1 / 2} \nabla_{h} v\left\|_{\left[L^{2}(\Omega)\right]^{d}}^{2}+|v|_{\mathrm{J}, \boldsymbol{\kappa}}^{2}, \quad\right\|\|v\|_{\boldsymbol{\kappa}, *}^{2}:=\|v\|_{\boldsymbol{\kappa}}^{2}+\sum_{T \in \mathcal{T}_{h}} h_{T}\left\|\left(\boldsymbol{\kappa}^{1 / 2} \nabla v\right)_{\mid T} \cdot \mathbf{n}_{T}\right\|_{L^{2}(\partial T)}^{2},\right.\right.
$$

where the jump seminorm is given by

$$
|v|_{\mathrm{J}, \kappa}^{2}:=\sum_{F \in \mathcal{F}_{h}} \frac{\gamma_{F}}{h_{F}}\|\llbracket v \rrbracket\|_{L^{2}(F)}^{2}
$$

Observe that the seminorm $|\cdot|_{\mathrm{J}, \boldsymbol{\kappa}}$ only depends on the diffusion coefficient $\boldsymbol{\kappa}$ via the stabilization parameter $\gamma_{F}$ defined by (3.5). The following lemma contains some important properties of the bilinear form $a_{h}$, namely consistency, coercivity and boundedness. We emphasize, in particular, the role of the choices (3.3) and (3.5) in delivering coercivity and boundedness constant that are independent of the diffusion coefficient $\boldsymbol{\kappa}$.

Lemma 3.3 (properties of the bilinear form $a_{h}$ ). The bilinear form $a_{h}$ enjoys the following properties:

(i) consistency. Assume $u \in V_{*}$. Then, for all $v_{h} \in V_{h}^{\mathrm{ccg}}$,

$$
a_{h}\left(u, v_{h}\right)=\int_{\Omega} f v_{h}
$$

(ii) coercivity. For all $\eta>\underline{\eta}:=C_{\mathrm{tr}}^{2} N_{\partial}$ there holds

$$
\forall v_{h} \in V_{h}^{\mathrm{ccg}}, \quad a_{h}\left(v_{h}, v_{h}\right) \geq C_{\text {sta }}\left\|v_{h}\right\|_{\kappa}^{2},
$$

with $C_{\text {sta }}:=\left(\eta-C_{\mathrm{tr}}^{2} N_{\partial}\right)\left\{\max \left(1 / 2, \eta+C_{\mathrm{tr}}^{2} N_{\partial}\right)\right\}^{-1}$ independent of both $\boldsymbol{\kappa}$ and $h$;

(iii) boundedness. There is $C_{\mathrm{bnd}}$ independent of the meshsize $h$ and of the diffusion coefficient $\boldsymbol{\kappa}$ such that

$$
\forall\left(v, w_{h}\right) \in V_{* h} \times V_{h}^{\mathrm{ccg}}, \quad a_{h}\left(v, w_{h}\right) \leq C_{\mathrm{bnd}}\left\|\left|v\|\|_{\kappa, *}\right|\right\| w_{h} \mid \|_{\kappa} .
$$

Proof. We preliminarily note the following bound resulting from the Cauchy-Schwarz inequality:

$$
\forall\left(v, w_{h}\right) \in V_{* h} \times V_{h}^{\mathrm{ccg}}, \quad\left|\sum_{F \in \mathcal{F}_{h}} \int_{F}\left\{\boldsymbol{\kappa} \nabla_{h} v\right\}_{\omega} \cdot \mathbf{n}_{F} \llbracket w_{h} \rrbracket\right| \leq\left(\sum_{T \in \mathcal{T}_{h}} \sum_{F \in \mathcal{F}_{T}} h_{F}\left\|\left(\boldsymbol{\kappa}^{1 / 2} \nabla v\right)_{\mid T} \cdot \mathbf{n}_{F}\right\|_{L^{2}(F)}^{2}\right)^{1 / 2}\left|w_{h}\right|_{\mathrm{J}, \boldsymbol{\kappa}}
$$

Consistency. Plugging the exact solution $u$ into the first argument of $a_{h}$ and integrating by parts we obtain, for all $v_{h} \in V_{h}^{\mathrm{ccg}}$,

$$
a_{h}\left(u, v_{h}\right)=-\int_{\Omega} \nabla \cdot(\boldsymbol{\kappa} \nabla u) v_{h}+\sum_{F \in \mathcal{F}_{h}^{\mathrm{i}}} \int_{F} \llbracket \boldsymbol{\kappa} \nabla_{h} u \rrbracket \cdot \mathbf{n}_{F}\left\{v_{h}\right\} \bar{\omega}-\sum_{F \in \mathcal{F}_{h}} \int_{F} \llbracket u \rrbracket\left\{\boldsymbol{\kappa} \nabla_{h} v_{h}\right\}_{\omega} \cdot \mathbf{n}_{F}+\sum_{F \in \mathcal{F}_{h}} \eta \frac{\gamma_{F}}{h_{F}} \int_{F} \llbracket u \rrbracket \llbracket v_{h} \rrbracket
$$

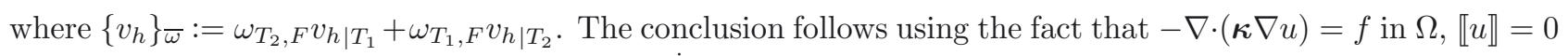
for all $F \in \mathcal{F}_{h}$ and $\llbracket \kappa \nabla u \rrbracket \cdot \mathbf{n}_{F}=0$ for all $F \in \mathcal{F}_{h}^{\mathrm{i}}$.

Coercivity. It is inferred from the bound (3.8) together with the discrete trace inequality (2.4) that

$$
\forall v_{h} \in V_{h}^{\mathrm{ccg}}, \quad\left|\sum_{F \in \mathcal{F}_{h}} \int_{F}\left\{\boldsymbol{\kappa} \nabla_{h} v_{h}\right\}_{\omega} \cdot \mathbf{n}_{F} \llbracket v_{h} \rrbracket\right| \leq C_{\mathrm{tr}} N_{\partial}^{1 / 2}\left\|\boldsymbol{\kappa}^{1 / 2} \nabla_{h} v_{h}\right\|_{\left[L^{2}(\Omega)\right]^{d}}\left|v_{h}\right|_{\mathrm{J}, \boldsymbol{\kappa}} .
$$


Using the inequality $2 a b \leq \epsilon a^{2}+1 / \epsilon b^{2}$ valid for any $\epsilon>0$ together with the above bound, we obtain

$$
\begin{aligned}
a_{h}\left(v_{h}, v_{h}\right) & =\left\|\boldsymbol{\kappa}^{1 / 2} \nabla_{h} v_{h}\right\|_{\left[L^{2}(\Omega)\right]^{d}}^{2}-2 \sum_{F \in \mathcal{F}_{h}} \int_{F}\left\{\boldsymbol{\kappa} \nabla_{h} v_{h}\right\}_{\omega} \cdot \mathbf{n}_{F} \llbracket v_{h} \rrbracket+\eta\left|v_{h}\right|_{\mathrm{J}, \boldsymbol{\kappa}}^{2} \\
& \geq\left(1-C_{\mathrm{tr}}^{2} N_{\partial} \epsilon\right)\left\|\boldsymbol{\kappa}^{1 / 2} \nabla_{h} v_{h}\right\|_{\left[L^{2}(\Omega)\right]^{d}}^{2}+(\eta-1 / \epsilon)\left|v_{h}\right|_{\mathrm{J}, \boldsymbol{\kappa}}^{2} .
\end{aligned}
$$

The desired result follows by properly selecting $\epsilon$.

Boundedness. Let $\left(v, w_{h}\right) \in V_{* h} \times V_{h}^{\text {ccg }}$ and let $\mathfrak{T}_{1}, \ldots, \mathfrak{T}_{4}$ denote the addends in the expression of $a_{h}\left(v, w_{h}\right)$ obtained from (3.4). Using the Cauchy-Schwarz inequality it is readily inferred that $\left|\mathfrak{T}_{1}+\mathfrak{T}_{4}\right| \leq(1+\eta)|| v\left|\left\|_{\kappa}\right\|\right| w_{h} \mid \|_{\kappa}$. Moreover, owing to the bound (3.8), $\left|\mathfrak{T}_{2}\right| \leq\left|\left\|\left.v\left|\|_{\boldsymbol{\kappa}, *}\right| w_{h}\right|_{\mathrm{J}, \boldsymbol{\kappa}}\right.\right.$. Finally, $\left.\left|\mathfrak{T}_{3}\right| \leq C_{\mathrm{tr}} N_{\partial}^{1 / 2}|v|_{\mathrm{J}, \boldsymbol{\kappa}}\right\| \boldsymbol{\kappa}^{1 / 2} \nabla_{h} w_{h} \|_{\left[L^{2}(\Omega)\right]^{d}}$, and the conclusion follows.

The following result classically follows from Lemma 3.3.

Theorem $3.4\left(|||\cdot| \|_{\kappa}\right.$-error estimate). Let $u \in H_{0}^{1}(\Omega)$ solve $(3.1)$ and assume $u \in V_{*}$. Let, moreover, $\left(u_{h}\right)_{h \in \mathcal{H}}$ denote the sequence of discrete solution to problem (3.6) on the admissible mesh sequence $\mathcal{T}_{\mathcal{H}}$. Then,

$$
\left\|u-u_{h}\left|\left\|_{\kappa} \leq\left(1+\frac{C_{\text {bnd }}}{C_{\text {sta }}}\right) \inf _{v_{h} \in V_{h}^{\text {ccg }}} \mid\right\| u-v_{h} \|_{\kappa, *}\right.\right.
$$

An important remark is that the error estimate in Theorem 3.4 is robust in that the multiplicative constant in the right-hand side does not depend on $\boldsymbol{\kappa}$. To infer a convergence rate from Theorem 3.4 we have to study the approximation properties of the discrete space $V_{h}^{\mathrm{ccg}}$ and further bound inf $v_{v_{h} \in V_{h}^{\mathrm{ccg}}}\left\|u-v_{h} \mid\right\| \|_{\kappa, *}$.

\subsection{Convergence rate}

\subsubsection{The heterogeneous case}

We first consider the heterogeneous case and focus on exact solutions exhibiting further local regularity. For $k \geq 0$ define the spaces of piecewise regular functions

$$
C^{k}\left(\mathcal{T}_{h}\right):=\left\{v \in L^{2}(\Omega) \mid v_{\mid T} \in C^{k}(\bar{T}), \forall T \in \mathcal{T}_{h}\right\}
$$

classically equipped with the norm

$$
\|v\|_{C^{k}\left(\mathcal{T}_{h}\right)}:=\max _{T \in \mathcal{T}_{h}} \max _{0 \leq l \leq k, 1 \leq i \leq d}\left\|\partial_{i}^{l} v\right\|_{C^{0}(\bar{T})},
$$

and $\|w\|_{C^{0}(\bar{T})}:=\max _{x \in \bar{T}}|w(x)|$ for all $w \in C^{0}(\bar{T})$. A proof of the following result is given in [4], Appendix A.

Theorem 3.5 (space $\left.\mathcal{Q}_{P_{\Omega}, \kappa}\right)$. Let $\mathcal{Q}_{P_{\Omega}, \kappa}$ be the space of functions $\varphi: \bar{\Omega} \rightarrow \mathbb{R}$ enjoying the following properties:

(i) global and local regularity. The function $\varphi$ belongs to $C_{0}(\bar{\Omega}) \cap C^{2}\left(P_{\Omega}\right)$ with $C^{2}\left(P_{\Omega}\right)$ spanned by the functions that are $C^{2}(\bar{S})$ for all $S \in P_{\Omega}$;

(ii) continuity of the tangential derivatives at interfaces. For all $S_{1}, S_{2} \in P_{\Omega}$ with intersection $\varsigma:=\partial S_{1} \cap \partial S_{2}$ such that $|\varsigma|_{d-1}>0$ and all vector $\mathbf{t}$ parallel to $\varsigma$,

$$
\nabla \varphi_{\mid S_{1}} \cdot \mathbf{t}=\nabla \varphi_{\mid S_{2}} \cdot \mathbf{t} \quad \text { on } \varsigma
$$

(iii) continuity of the diffusive flux at interfaces. For all $S_{1}, S_{2} \in P_{\Omega}$ with intersection $\varsigma:=\partial S_{1} \cap \partial S_{2}$ such that $|\varsigma|_{d-1}>0$,

$$
(\kappa \nabla \varphi)_{\mid S_{1}} \cdot \mathbf{n}_{\varsigma}=(\kappa \nabla \varphi)_{\mid S_{2}} \cdot \mathbf{n}_{\varsigma}, \quad \text { on } \varsigma,
$$

where $\mathbf{n}_{\varsigma}$ is the outer normal to $S_{1}$.

Then, $\mathcal{Q}_{P_{\Omega}, \boldsymbol{\kappa}}$ is dense in $H_{0}^{1}(\Omega)$. 
An important remark is that $\mathcal{Q}_{P_{\Omega}, \kappa}$ is a subspace of $V_{*}$, and so the assumptions required to prove the error estimate in Theorem 3.4 are met by solutions that are in $\mathcal{Q}_{P_{\Omega}, \kappa}$. Moreover, it is clear from Assumption 2.9 that $\mathcal{Q}_{P_{\Omega}, \kappa} \subset C^{2}\left(\mathcal{T}_{h}\right)$. In what follows we denote by $\mathcal{I}_{h}^{\text {ccg }}: C^{0}\left(\mathcal{T}_{h}\right) \rightarrow \mathbb{V}_{h}$ the interpolator at cell centers which maps every function $v \in C^{0}\left(\mathcal{T}_{h}\right)$ onto the vector

$$
\mathcal{I}_{h}^{\mathrm{ccg}}(v)=\left(v\left(\mathbf{x}_{T}\right)\right)_{T \in \mathcal{T}_{h}} \in \mathbb{V}_{h}
$$

The following result is an immediate consequence of [4], Lemma 3.3, together with the shape- and contactregularity of the mesh family $\mathcal{T}_{\mathcal{H}}$.

Lemma 3.6 (consistency of group gradients). For all $h \in \mathcal{H}$, all $v \in \mathcal{Q}_{P_{\Omega}, \kappa}$, and all $F \in \mathcal{F}_{h}$,

$$
\left|\nabla \xi_{\mathbf{v}_{h}}^{\mathfrak{g}_{F}}-\nabla v\left(\mathbf{x}_{T_{\mathfrak{g}_{F}}}\right)\right| \leq C\left(1+\left\|\mathbf{A}_{\mathfrak{g}_{F}}^{-1}\right\|_{2}\right) h_{T_{\mathfrak{g}_{F}}}
$$

where $\mathbf{v}_{h}=\mathcal{I}_{h}^{\mathrm{ccg}}(v)$ and $C$ depends on $\boldsymbol{\kappa}$, on the mesh regularity parameters, and on $\|v\|_{C^{2}\left(\mathcal{I}_{h}\right)}$ but not on $h$.

In view of Lemma 3.6, we need to ensure first-order convergence of the group gradients, which requires the following assumption.

Assumption 3.7 (uniform bound on $\left\|\mathbf{A}_{\mathfrak{g}_{F}}^{-1}\right\|_{2}$ ). We assume that there exists $\Lambda<+\infty$ independent of the meshsize $h$ uniformly bounding the set $\left\{\left\|\mathbf{A}_{\mathfrak{g}_{F}}^{-1}\right\|_{2}\right\}_{h \in \mathcal{H}, F \in \mathcal{F}_{h}^{\mathrm{i}}}$ from above.

For further use we introduce the following augmented version of the $\||\cdot| \mid-$-norm on $V_{*}$ :

$$
\left\|v\left|\left\|_{*}^{2}:=\right\| v\right|\right\|^{2}+\sum_{T \in \mathcal{T}_{h}} h_{T}\left\|\nabla v_{\mid T} \cdot \mathbf{n}_{T}\right\|_{L^{2}(\partial T)}^{2} .
$$

The proof of the following result, which is instrumental in estimating the convergence rate, is given in Section A.1.

Theorem 3.8 (approximation of functions in $\left.\mathcal{Q}_{P_{\Omega}, \kappa}\right)$. Let $v \in \mathcal{Q}_{P_{\Omega}, \kappa}$ and set $v_{h}:=\left(\Re_{h}^{\mathrm{ccg}} \circ \mathcal{I}_{h}^{\mathrm{ccg}}\right)(v) \in V_{h}^{\mathrm{ccg}}$. Then, under Assumption 3.7 there holds

$$
\left\|v-v_{h}\right\|_{\kappa, *} \leq \bar{\lambda}^{1 / 2}\left\|v-v_{h}\right\|_{*} \leq C_{v} h
$$

with $C_{v}$ depending on $\boldsymbol{\kappa}$, on the mesh regularity parameters, on $\Lambda$, and on $\|v\|_{C^{2}\left(\mathcal{T}_{h}\right)}$ but independent of the meshsize $h$.

Corollary 3.9 (convergence rate, heterogeneous case). Assume $u \in \mathcal{Q}_{P_{\Omega}, \kappa}$. Then, under Assumption 3.7, there holds

$$
\left\|u-u_{h}\right\|_{\kappa} \leq C h
$$

with $C=C_{u} \bar{\lambda}^{1 / 2}\left(1+\frac{C_{\mathrm{bnd}}}{C_{\mathrm{sta}}}\right)$ and $C_{u}$ results from Theorem 3.8. C additionally depends on the mesh regularity parameters but is independent of the meshsize $h$.

\subsubsection{The homogeneous isotropic case}

Whenever the diffusion coefficient is homogeneous and isotropic, the above results can be further refined. We assume throughout this section that

$$
\boldsymbol{\kappa}=\mathbf{1}_{d}, \quad d \in\{2,3\} .
$$

The more general case $\boldsymbol{\kappa}=\nu \mathbf{1}_{d}, \nu>0$, can be handled with minor modifications. When (3.10) holds, convergence rates can be estimated with milder regularity assumptions on the exact solution, and $L^{2}$-error estimates for the method (3.6) can be obtained by the Aubin-Nietsche trick [8,41]. The key point is here to show how optimal convergence rates can be obtained avoiding pointwise estimations, thereby removing the need for the strong local regularity assumption $u \in C^{2}\left(\mathcal{T}_{h}\right)$ used all along the previous section. To this end, we need to 
introduce further mild assumptions on the mesh family. For all faces we define a patch of pyramids on which the piecewise affine functions $\left\{\xi_{\mathbf{v}_{h}}^{\mathfrak{g}_{F}}\right\}_{F \in \mathcal{F}_{h}}$ are required to exhibit approximation properties. More precisely, for all $F \in \mathcal{F}_{h}$ let

$$
\mathcal{P}_{F}:= \begin{cases}\mathcal{P}_{\mathfrak{g}_{F}} & \text { if } F \in \mathcal{F}_{h}^{\mathrm{i}}, \\ \mathcal{P}_{T, F} & \text { if } F \subset \partial T \cap \partial \Omega,\end{cases}
$$

with $\mathcal{P}_{\mathfrak{g}_{F}}$ defined by (2.10); see Figure 1.

Assumption 3.10 (approximation property for L-constructions). We assume that the L-constructions are such that there exists $C$ independent of the meshsize $h$ such that, for all $F \in \mathcal{F}_{h}$ and all $v \in H^{2}(\Omega) \cap H_{0}^{1}(\Omega)$,

$$
\left\|v-\xi_{\mathbf{v}_{h}}^{\mathfrak{g}_{F}}\right\|_{L^{2}\left(\mathcal{P}_{F}\right)}+h_{\mathcal{P}_{F}}\left|v-\xi_{\mathbf{v}_{h}}^{\mathfrak{g}_{F}}\right|_{H^{1}\left(\mathcal{P}_{F}\right)} \leq C h_{\mathcal{P}_{F}}^{2}|v|_{H^{2}\left(\mathcal{P}_{F}\right)}
$$

with $\mathbf{v}_{h}=\mathcal{I}_{h}^{\mathrm{ccg}}(v)$. Moreover, for all $F \in \mathcal{F}_{h}$ and all $T \in \mathcal{T}_{F}, h_{\mathcal{P}_{F}}$ is bounded by $h_{T}$ uniformly with respect to $h$.

Some comments are of order. We start by observing that Assumption 2.11 has a straightforward interpretation in the homogeneous case, as it amounts to requiring that, for all $F \in \mathcal{F}_{h}^{\mathrm{i}}$, there exists at least a group $\mathfrak{g} \in$ $\mathcal{G}_{F}$ such that the cell centers of the elements in $\mathcal{T}_{\mathfrak{g}}$ and the barycenters of the faces $F \in \mathfrak{g}^{\mathrm{b}}$ form a nondegenerate simplex $S_{\mathfrak{g}}$; see Figure 1. Assumption 3.7 is then essentially a shape-regularity requirement on the family of simplices $\left\{S_{\mathfrak{g}_{F}}\right\}_{h \in \mathcal{H}, F \in \mathcal{F}_{h}^{\mathrm{i}}}$. A second important remark is that, for any function $v$ regular enough, the function $\xi_{\mathbf{v}_{h}}^{\mathfrak{g}}$ with $\mathbf{v}_{h}=\mathcal{I}_{h}^{\text {ccg }}(v)$ coincides with the Lagrange interpolator on $S_{\mathfrak{g}}$ (a sufficient regularity is $v \in H^{2}(\Omega)$ for $d \in\{2,3\}$; see, e.g., [30], Ex. 1.106). In this respect, Assumption 3.10 can be regarded as a strengthening of Assumption 3.7. Indeed, Assumption 3.10 can be proven using classical FE techniques in some cases provided sufficient (geometric) regularity holds. A simple example is provided by Cartesian orthogonal grids with nonconforming local refinement and bounded difference in refinement level between neighbouring elements. As a matter of fact, in this case we have a finite number of reference configurations onto which the patches used in the L-construction can be mapped via an affine bijective mapping, and one can classically proceed using the Deny-Lions Lemma. We also emphasize that Assumption 3.10 does not necessarily follow from the approximation properties of the mesh sequence $\mathcal{T}_{\mathcal{H}}$, since the reconstruction is grounded on the patches $\mathcal{P}_{F}$, $F \in \mathcal{F}_{h}$, and not on the elements of $\mathcal{T}_{h}$. The proof of the following technical result is postponed to Section A.2.

Theorem 3.11 (approximation of functions in $V \cap H^{2}(\Omega)$ ). Under Assumption 3.10, there holds

$$
\forall v \in V \cap H^{2}(\Omega), \quad\|\| v-v_{h}\left\|_{*} \leq C h\right\| v \|_{H^{2}(\Omega)},
$$

with $v_{h}:=\left(\Re_{h}^{\mathrm{ccg}} \circ \mathcal{I}_{h}^{\mathrm{ccg}}\right)(v) \in V_{h}^{\mathrm{ccg}}$ and $C$ depending on the mesh regularity parameters but not on $h$.

Definition 3.12 (elliptic regularity). We say that elliptic regularity holds true if there is $C_{\text {ell }}$, depending only on the domain $\Omega$, such that, for all $\psi \in L^{2}(\Omega)$, the solution to the following problem:

$$
\text { Find } \chi \in H_{0}^{1}(\Omega) \text { s.t. } a(\chi, v)=\int_{\Omega} \psi v \text { for all } v \in H_{0}^{1}(\Omega)
$$

with $a(w, v):=\int_{\Omega} \nabla w \cdot \nabla v$ satisfies $\|\chi\|_{H^{2}(\Omega)} \leq C_{\mathrm{ell}}\|\psi\|_{L^{2}(\Omega)}$.

Elliptic regularity can be asserted if, for instance, the polygonal domain $\Omega$ is convex; see Grisvard [35].

Lemma 3.13 (convergence rate, homogeneous case). Let $u \in V$ be the unique solution to (3.1) and assume elliptic regularity. Then, under Assumption 3.10, there exists $C$ independent of the meshsize $h$ such that

$$
\begin{aligned}
\left\|u-u_{h}\right\| & \leq C h\|u\|_{H^{2}(\Omega)}, \\
\left\|u-u_{h}\right\|_{L^{2}(\Omega)} & \leq C h^{2}\|u\|_{H^{2}(\Omega)}
\end{aligned}
$$


Proof. (i) Energy-norm estimate (3.12). Use the error estimate of Theorem 3.4 together with Theorem 3.11 and the fact that the ||$|\cdot| \|_{\kappa^{-}}$and $\mid\|\cdot\| \|_{\kappa, *}$-norms coincide with the ||$|\cdot|||$ - and ||$\cdot \mid \|_{*}$-norms respectively under assumption (3.10) to conclude that $\left\|u-u_{h}\right\|_{\kappa} \leq C\left(1+\frac{C_{\text {bnd }}}{C_{\text {sta }}}\right)\|v\|_{H^{2}(\Omega)}$.

(ii) $L^{2}$-error estimate (3.13). We only give a sketch of the proof and refer, e.g., to [7] or [24], Chapter 4, for further details. A preliminary step consists in estimating the error in the augmented norm $\|\mid \cdot\| \|_{*}$. To this end, remark that the bilinear form $a_{h}$ is coercive in $V_{h}^{\mathrm{ccg}} \times V_{h}^{\mathrm{ccg}}$ with respect to the augmented norm $\|\cdot\|_{*}$, i.e., there exists $C_{\text {sta }}^{\prime}$ such that, for all $\eta>\underline{\eta}$ and all $v_{h} \in V_{h}^{\text {ccg }}, a_{h}\left(v_{h}, v_{h}\right) \geq C_{\text {sta }}^{\prime}\left\|v_{h}\right\| \|_{*}^{2}$ (indeed, the $\|\mid\|$-norm and the $\|\cdot\| \|_{*}$-norm are uniformly equivalent on $V_{h}^{\mathrm{ccg}}$ ). Also, there exists $C_{\mathrm{bnd}}^{\prime}$ independent of the meshsize $h$ such that, for all $w, v \in V_{* h}, a_{h}(w, v) \leq C_{\mathrm{bnd}}^{\prime}\|w\|\left\|_{*}\right\| \mid v \|_{*}$. Hence, proceeding as in Theorem 3.4 and using Theorem 3.11 we conclude that $\left\|u-u_{h}\right\|_{*} \leq C h\|u\|_{H^{2}(\Omega)}$ with $C$ independent of the meshsize $h$. Consider now the auxiliary problem (3.11) with $\psi=u-u_{h}$. By the elliptic regularity assumption, there exists $C_{\text {ell }}$ such that $\|\chi\|_{H^{2}(\Omega)} \leq C_{\text {ell }}\left\|u-u_{h}\right\|_{L^{2}(\Omega)}$. Moreover, owing to the symmetry and the consistency of $a_{h}$, $a_{h}\left(u-u_{h}, \chi\right)=-\int_{\Omega} \triangle \chi\left(u-u_{h}\right)$ and, for $\chi_{h}=\left(\Re_{h}^{\mathrm{ccg}} \circ \mathcal{I}_{h}^{\mathrm{ccg}}\right)(\chi) \in V_{h}^{\mathrm{ccg}}, a_{h}\left(u-u_{h}, \chi_{h}\right)=0$. As a result,

$\left\|u-u_{h}\right\|_{L^{2}(\Omega)}^{2}=a_{h}\left(u-u_{h}, \chi-\chi_{h}\right) \lesssim\left\|u-u_{h}\right\|_{*}\left\|\chi \chi-\chi_{h}\left|\left\|_{*} \lesssim\right\| u-u_{h}\right|\right\|\left\|_{*} h\right\| \chi\left\|_{H^{2}\left(\mathcal{T}_{h}\right)} \lesssim\right\| u-u_{h}\|\|_{*} h\left\|u-u_{h}\right\|_{L^{2}(\Omega)}$,

with $\lesssim$ indicating inequalities up to a multiplicative constant independent of the meshsize $h$. To conclude the proof, use the fact that $\left\|u-u_{h}\right\|\left\|_{*} \leq C h\right\| v \|_{H^{2}(\Omega)}$.

\subsection{Convergence to minimal regularity solutions}

We investigate the convergence of the method (3.6) to minimal regularity solutions, i.e., solutions that barely sit in $H_{0}^{1}(\Omega)$. Throughout this section we restore the original assumptions on the diffusion coefficient $\boldsymbol{\kappa}$, and we consider an arbitrary space dimension, i.e.

$$
\kappa \in\left[\mathbb{P}_{d}^{0}\left(\mathcal{T}_{h}\right)\right]^{d, d}, \quad d \geq 2 .
$$

The analysis follows the ideas of Eymard et al. [32,33] originally developed in the context of FV methods and recently transposed to $\mathrm{dG}$ methods by Di Pietro and Ern [22]. An important remark is that the bilinear form $a_{h}$ admits the following equivalent form on $V_{h}^{\mathrm{ccg}} \times V_{h}^{\mathrm{ccg}}$ :

$$
a_{h}\left(u_{h}, v_{h}\right)=\int_{\Omega} \kappa \mathbf{G}_{\omega, h}\left(u_{h}\right) \cdot \mathbf{G}_{\omega, h}\left(v_{h}\right)+j_{h}\left(u_{h}, v_{h}\right)
$$

with $j_{h}\left(u_{h}, v_{h}\right):=-\int_{\Omega} \boldsymbol{\kappa} \mathbf{R}_{\omega, h}\left(\llbracket u_{h} \rrbracket\right) \cdot \mathbf{R}_{\omega, h}\left(\llbracket v_{h} \rrbracket\right)+\sum_{F \in \mathcal{F}_{h}} \eta \gamma_{F} h_{F}^{-1} \int_{F} \llbracket u_{h} \rrbracket \llbracket v_{h} \rrbracket$ and discrete gradients defined by (2.17). When extended to $V_{* h} \times V_{h}^{\text {ccg }}$, this alternative form is no longer consistent in the sense of point (i) in Lemma 3.3; see [22], Remark 3.3. However, $a_{h}$ retains a different form of consistency which suffices to infer the convergence of the method when $u$ only exhibits the minimal regularity.

Definition 3.14 (asymptotic consistency). We say that the bilinear form $a_{h}$ is asymptotically consistent with the exact bilinear form $a$ on $V_{\mathcal{H}}^{\text {ccg }}$ if, for any sequence $\left(v_{h}\right)_{h \in \mathcal{H}}$ in $V_{\mathcal{H}}^{\text {ccg }}$ uniformly bounded in the $\|\cdot\| \|$-norm, and for all $\varphi \in \mathcal{Q}_{P_{\Omega}, \kappa}$ with $\varphi_{h}:=\left(\mathfrak{R}_{h}^{\mathrm{ccg}} \circ \mathcal{I}_{h}^{\mathrm{ccg}}\right)(\varphi) \in V_{h}^{\mathrm{ccg}}$,

$$
\lim _{h \rightarrow 0} a_{h}\left(v_{h}, \varphi_{h}\right)=a(v, \varphi)=\int_{\Omega} \kappa \nabla v \cdot \nabla \varphi,
$$

and $v \in H_{0}^{1}(\Omega)$ results from Theorem 2.15.

To prove the convergence of the method, we then proceed as follows: (i) using the coercivity of $a_{h}$ we prove a uniform bound for the ||$|\cdot| \mid-$-norm of the discrete solutions $\left(u_{h}\right)_{h \in \mathcal{H}}$; (ii) by virtue of Theorem 2.15 , we infer the existence of $\bar{u} \in V=H_{0}^{1}(\Omega)$ limit of $\left(u_{h}\right)_{h \in \mathcal{H}}$; (iii) using the asymptotic consistency of $a_{h}$ together with the 
fact that $\mathcal{Q}_{P_{\Omega}, \kappa}$ is dense in $V$, we conclude that $\bar{u}=u$ with $u$ solution to (3.1) (and, by the uniqueness of $u$, that the convergence property extends to the whole sequence); (iv) using the above result, we prove the strong convergence of the sequence $\left(\mathbf{G}_{\omega, h}\left(u_{h}\right)\right)_{h \in \mathcal{H}}$ to $\nabla u$ in $\left[L^{2}(\Omega)\right]^{d}$ as $h \rightarrow 0$. An important intermediate result to prove the asymptotic consistency of $a_{h}$ is the consistency of the discrete gradient $\mathbf{G}_{\omega, h}$ defined by (2.17) for the interpolates of functions in $\mathcal{Q}_{P_{\Omega}, \kappa}$. We first prove the following.

Lemma 3.15 (bound on global lifting). For all $v \in V_{*}$, there holds

$$
\left\|\boldsymbol{\kappa}^{1 / 2} \mathbf{R}_{\omega, h}(\llbracket v \rrbracket)\right\|_{\left[L^{2}(\Omega)\right]^{d}} \leq C_{\mathrm{tr}} N_{\partial}^{1 / 2}|v|_{J, \kappa} .
$$

Proof. By definition,

$$
\left\|\boldsymbol{\kappa}^{1 / 2} \mathbf{R}_{\omega, h}(\llbracket v \rrbracket)\right\|_{\left[L^{2}(\Omega)\right]^{d}}^{2}=\sum_{F \in \mathcal{F}_{h}} \int_{\Omega} \boldsymbol{\kappa} \mathbf{R}_{\omega, h}(\llbracket v \rrbracket) \cdot \mathbf{r}_{\omega, F}(\llbracket v \rrbracket)=\sum_{F \in \mathcal{F}_{h}} \int_{F}\left\{\boldsymbol{\kappa} \mathbf{R}_{\omega, h}\left(\llbracket v_{h} \rrbracket\right)\right\}_{\omega} \cdot \mathbf{n}_{F} \llbracket v \rrbracket .
$$

For brevity of notation, for all $F \in \mathcal{F}_{h}^{\mathrm{i}}$ with $F=\partial T_{1} \cap \partial T_{2}$, let $\omega_{i}=\omega_{T_{i}, F}, \lambda_{T_{i}, F}=\lambda_{i}, \boldsymbol{\kappa}_{i}=\boldsymbol{\kappa}_{\mid T_{i}}$, and $\mathbf{a}_{i}=\boldsymbol{\kappa}_{i}^{1 / 2} \mathbf{R}_{\omega, h}(\llbracket v \rrbracket)_{\mid T_{i}}, i \in\{1,2\}$. The Cauchy-Schwarz inequality yields

$$
\begin{aligned}
\int_{F}\left\{\boldsymbol{\kappa} \mathbf{R}_{\omega, h}(\llbracket v \rrbracket)\right\}_{\omega} \cdot \mathbf{n}_{F} \llbracket v \rrbracket & =\int_{F}\left(\omega_{1} \boldsymbol{\kappa}_{1}^{1 / 2} \mathbf{n}_{T_{1}} \cdot \mathbf{a}_{1}+\omega_{2} \boldsymbol{\kappa}_{2}^{1 / 2} \mathbf{n}_{T_{2}} \cdot \mathbf{a}_{2}\right) \llbracket v \rrbracket \\
& \leq\left\{\frac{1}{2} h_{F}\left(\left\|\mathbf{a}_{1}\right\|_{\left[L^{2}(F)\right]^{d}}^{2}+\left\|\mathbf{a}_{2}\right\|_{\left[L^{2}(F)\right]^{d}}^{2}\right)\right\}^{1 / 2} \times\left\{2\left(\omega_{1}^{2} \lambda_{1}+\omega_{2}^{2} \lambda_{2}\right) \frac{1}{h_{F}}\|\llbracket v \rrbracket\|_{L^{2}(F)}^{2}\right\}^{1 / 2},
\end{aligned}
$$

and since $2\left(\omega_{1}^{2} \lambda_{1}+\omega_{2}^{2} \lambda_{2}\right)=\gamma_{F}$, it is inferred that

$$
\int_{F}\left\{\boldsymbol{\kappa} \mathbf{R}_{\omega, h}(\llbracket v \rrbracket)\right\}_{\omega} \cdot \mathbf{n}_{F} \llbracket v \rrbracket \leq\left\{\frac{1}{2} h_{F}\left(\left\|\mathbf{a}_{1}\right\|_{\left[L^{2}(F)\right]^{d}}^{2}+\left\|\mathbf{a}_{2}\right\|_{\left[L^{2}(F)\right]^{d}}^{2}\right)\right\}^{1 / 2} \times\left\{\frac{\gamma_{F}}{h_{F}}\|\llbracket v \rrbracket\|_{L^{2}(F)}^{2}\right\}^{1 / 2} .
$$

Moreover, for all $F \in \mathcal{F}_{h}^{\mathrm{b}}$ with $F=\partial T \cap \partial \Omega$,

$$
\int_{F}\left\{\boldsymbol{\kappa} \mathbf{R}_{\omega, h}(\llbracket v \rrbracket)\right\}_{\omega} \cdot \mathbf{n}_{F} \llbracket v \rrbracket \leq h_{F}^{1 / 2}\left\|\left(\boldsymbol{\kappa}^{1 / 2} \mathbf{R}_{\omega, h}(\llbracket v \rrbracket)\right)_{\mid T} \cdot \mathbf{n}_{F}\right\|_{L^{2}(F)} \times\left\{\frac{\gamma_{F}}{h_{F}}\|\llbracket v \rrbracket\|_{L^{2}(F)}^{2}\right\}^{1 / 2} .
$$

Summing over mesh faces, and using the Cauchy-Schwarz and discrete trace inequality (2.4) we obtain

$$
\begin{aligned}
\left\|\boldsymbol{\kappa}^{1 / 2} \mathbf{R}_{\omega, h}(\llbracket v \rrbracket)\right\|_{\left[L^{2}(\Omega)\right]^{d}}^{2} & \leq \sum_{F \in \mathcal{F}_{h}}\left\{h_{F} \sum_{T \in \mathcal{T}_{F}}\left\|\left(\boldsymbol{\kappa}^{1 / 2} \mathbf{R}_{\omega, h}(\llbracket v \rrbracket)\right)_{\mid T}\right\|_{L^{2}(F)}^{2}\right\}^{1 / 2} \\
& \times|v|_{\mathrm{J}, \boldsymbol{\kappa}} \leq C_{\mathrm{tr}} N_{\partial}^{1 / 2}\left\|\boldsymbol{\kappa}^{1 / 2} \mathbf{R}_{\omega, h}(\llbracket v \rrbracket)\right\|_{\left[L^{2}(\Omega)\right]^{d}}|v|_{\mathrm{J}, \boldsymbol{\kappa}} .
\end{aligned}
$$

This concludes the proof.

Lemma 3.16 (strong convergence of $\mathbf{G}_{\omega, h}$ for smooth functions). Let $\varphi \in \mathcal{Q}_{P_{\Omega}, \kappa}$ and set $\varphi_{h}:=\left(\mathfrak{R}_{h}^{\mathrm{ccg}} \circ \mathcal{I}_{h}^{\mathrm{ccg}}\right)(\varphi) \in$ $V_{h}^{\mathrm{ccg}}$. Under Assumption 3.7, there holds

$$
\mathbf{G}_{\omega, h}\left(\varphi_{h}\right) \rightarrow \nabla \varphi \quad \text { strongly in }\left[L^{2}(\Omega)\right]^{d} .
$$

Proof. The triangular inequality yields

$$
\left\|\mathbf{G}_{\omega, h}\left(\varphi_{h}\right)-\nabla \varphi\right\|_{\left[L^{2}(\Omega)\right]^{d}} \leq\left\|\nabla_{h} \varphi_{h}-\nabla \varphi\right\|_{\left[L^{2}(\Omega)\right]^{d}}+\left\|\mathbf{R}_{\omega, h}\left(\llbracket \varphi_{h} \rrbracket\right)\right\|_{\left[L^{2}(\Omega)\right]^{d}}:=\mathfrak{T}_{1}+\mathfrak{T}_{2} .
$$


Using Theorem 3.8 it is readily inferred that $\mathfrak{T}_{1} \rightarrow 0$ as $h \rightarrow 0$. For the second term, use Lemma 3.15 together with the fact that $\llbracket \varphi \rrbracket_{F}=0$ on every $F \in \mathcal{F}_{h}$ to infer

$$
\left\|\boldsymbol{\kappa}^{1 / 2} \mathbf{R}_{\omega, h}\left(\llbracket \varphi_{h} \rrbracket\right)\right\|_{\left[L^{2}(\Omega)\right]^{d}} \leq C_{\mathrm{tr}} N_{\partial}^{1 / 2}\left|\varphi_{h}\right|_{\mathrm{J}, \kappa} \leq C_{\mathrm{tr}} N_{\partial}^{1 / 2}\left|\varphi_{h}-\varphi\right|_{\mathrm{J}, \kappa},
$$

and the right-hand side tends to zero as $h \rightarrow 0$ again by virtue of Theorem 3.8. This concludes the proof.

We are now ready to prove the following.

Lemma 3.17 (asymptotic consistency of the bilinear form $a_{h}$ ). Under Assumption 3.7, the bilinear form $a_{h}$ is asymptotically consistent with the exact bilinear form a on $V_{\mathcal{H}}^{\mathrm{ccg}}$.

Proof. Let $\left(v_{h}\right)_{h \in \mathcal{H}}$ be a sequence in $V_{\mathcal{H}}^{\mathrm{ccg}}$ bounded in the $\|\cdot\| \mid \|$-norm and let $\varphi \in \mathcal{Q}_{P_{\Omega}, \kappa}$. For all $h \in \mathcal{H}$, we set $\varphi_{h}:=\left(\Re_{h}^{\text {ccg }} \circ \mathcal{I}_{h}^{\text {ccg }}\right)(\varphi) \in V_{h}^{\text {ccg }}$. By Theorem 3.8, it is clear that $\left\|\varphi-\varphi_{h} \mid\right\|_{\kappa} \rightarrow 0$ as $h \rightarrow 0$. Observe that

$$
a_{h}\left(v_{h}, \varphi_{h}\right)=\int_{\Omega} \kappa \mathbf{G}_{\omega, h}\left(v_{h}\right) \cdot \mathbf{G}_{\omega, h}\left(\varphi_{h}\right)+j_{h}\left(v_{h}, \varphi_{h}\right):=\mathfrak{T}_{1}+\mathfrak{T}_{2}
$$

Clearly, as $h \rightarrow 0, \mathfrak{T}_{1} \rightarrow \int_{\Omega} \kappa \nabla v \cdot \nabla \varphi$ owing to the weak convergence of $\mathbf{G}_{\omega, h}\left(v_{h}\right)$ to $\nabla v$ stated in Lemma 2.16 and to the strong convergence of $\mathbf{G}_{\omega, h}\left(\varphi_{h}\right)$ to $\nabla \varphi$ proven in Lemma 3.16. Furthermore, using the CauchySchwarz inequality together with Lemma 3.15 and the fact that, for all $w \in H^{1}\left(\mathcal{T}_{h}\right),|w|_{\mathrm{J}, \boldsymbol{\kappa}} \leq \bar{\lambda}^{1 / 2}|w|_{\mathrm{J}}$, it is inferred that

$$
\left|\mathfrak{T}_{2}\right|=\left|j_{h}\left(v_{h}, \varphi_{h}\right)\right| \leq\left(C_{\mathrm{tr}}^{2} N_{\partial}+\eta\right)\left|v_{h}\right|_{\mathrm{J}, \kappa}\left|\varphi_{h}\right|_{\mathrm{J}, \kappa} \leq\left(C_{\mathrm{tr}}^{2} N_{\partial}+\eta\right) \bar{\lambda}^{1 / 2}\left|v_{h}\right|_{\mathrm{J}}\left|\varphi_{h}\right|_{\mathrm{J}, \kappa}
$$

Since $\left|v_{h}\right|_{\mathrm{J}}$ is bounded by assumption, and since $\left|\varphi_{h}\right|_{\mathrm{J}, \kappa}=\left|\varphi_{h}-\varphi\right|_{\mathrm{J}, \kappa}$ tends to zero as $h \rightarrow 0$, it is inferred that $\mathfrak{T}_{2} \rightarrow 0$. The proof is complete.

Remark 3.18 (weakening Assumption 3.7). To prove the asymptotic consistency of $a_{h}$, and hence the convergence to minimal regularity solutions, we only need that

$$
\forall \varphi \in \mathcal{Q}_{P_{\Omega}, \kappa}, \quad\|\| \varphi-\varphi_{h} \mid \| \rightarrow 0 \text { as } h \rightarrow 0,
$$

with $\varphi_{h}=\left(\mathfrak{R}_{h}^{\mathrm{ccg}} \circ \mathcal{I}_{h}^{\mathrm{ccg}}\right)(\varphi) \in V_{h}^{\mathrm{ccg}}$. Property (3.15) holds, e.g., if $\max _{F \in \mathcal{F}_{h}}\left\|\mathbf{A}_{\mathfrak{g}_{F}}^{-1}\right\|_{2} \leq C h^{-\epsilon}$ with $0 \leq \epsilon<1$ and $C$ independent of the meshsize $h$. Whenever the solution exhibits sufficient regularity, however, one may wish to have $\epsilon=0$ to attain optimal convergence rates. For the sake of simplicity, Assumption 3.7 is required in the statements of Lemma 3.17 and Theorem 3.19, although (3.15) could have been used instead.

Theorem 3.19 (convergence to minimal regularity solutions). Let $\left(u_{h}\right)_{h \in \mathcal{H}}$ be the sequence of approximate solutions generated by solving the discrete problems (3.6) on the mesh sequence $\mathcal{T}_{\mathcal{H}}$. Then, under Assumption 3.7 , for $\eta>\underline{\eta}$ with $\underline{\eta}$ defined in Lemma 3.3ii, as $h \rightarrow 0$, (i) $u_{h} \rightarrow u$ strongly in $L^{2}(\Omega)$, (ii) $\nabla_{h} u_{h} \rightarrow \nabla u$ strongly in $\left[L^{2}(\Omega)\right]^{\bar{d}}$, (iii) $\left|\bar{u}_{h}\right|_{\mathrm{J}} \rightarrow 0$, with $u \in V$ unique solution to $(3.2)$.

Proof. We follow the four steps outlined above.

(i) A priori estimate. Owing to Lemma 3.3ii and to the discrete Poincaré inequality obtained from (2.15) with $q=2$,

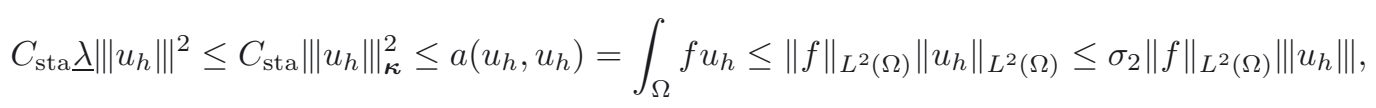

hence $\left\|u_{h}\right\| \mid \leq \sigma_{2}\left(C_{\eta} \underline{\lambda}\right)^{-1}\|f\|_{L^{2}(\Omega)}$.

(ii) Compactness. Owing to Theorem 2.15 together with Lemma 2.16, there exists $\bar{u} \in V=H_{0}^{1}(\Omega)$ such that, as $h \rightarrow 0$, up to a subsequence, $u_{h} \rightarrow \bar{u}$ strongly in $L^{2}(\Omega)$ and $\mathbf{G}_{\omega, h}\left(u_{h}\right) \rightarrow \nabla \bar{u}$ weakly in $\left[L^{2}(\Omega)\right]^{d}$. 
(iii) Identification of the limit. Owing to the asymptotic consistency of $a_{h}$ proven in Lemma 3.17, for all $\varphi \in \mathcal{Q}_{P_{\Omega}, \kappa}$ with $\varphi_{h}:=\left(\Re_{h}^{\mathrm{ccg}} \circ \mathcal{I}_{h}^{\mathrm{ccg}}\right)(\varphi) \in V_{h}^{\mathrm{ccg}}$,

$$
\int_{\Omega} f \varphi \leftarrow \int_{\Omega} f \varphi_{h}=a_{h}\left(u_{h}, \varphi_{h}\right) \rightarrow \int_{\Omega} \kappa \nabla \bar{u} \cdot \nabla \varphi
$$

i.e., $\bar{u}$ solves problem (3.2) by the density of $\mathcal{Q}_{P_{\Omega}, \kappa}$ in $V$ stated in Theorem 3.5 and, hence, $\bar{u}=u$. Moreover, since the solution $u$ to problem (3.2) is unique, the whole sequence converges (which is proven by contradiction). (iv) Strong convergence of the gradient and of the jumps. Lemma 3.15 and (3.14) yield

$$
\forall v_{h} \in V_{h}^{\mathrm{ccg}}, \quad a_{h}\left(v_{h}, v_{h}\right) \geq\left\|\boldsymbol{\kappa}^{1 / 2} \mathbf{G}_{\omega, h}\left(v_{h}\right)\right\|_{\left[L^{2}(\Omega)\right]^{d}}+\left(\eta-C_{\mathrm{tr}}^{2} N_{\partial}\right)\left|v_{h}\right|_{\mathrm{J}, \boldsymbol{\kappa}}^{2}
$$

Moreover, from the weak convergence of $\mathbf{G}_{\omega, h}\left(u_{h}\right)$ to $\nabla u$, we readily infer the weak convergence of $\boldsymbol{\kappa}^{1 / 2} \mathbf{G}_{\omega, h}\left(u_{h}\right)$ to $\boldsymbol{\kappa}^{1 / 2} \nabla u$. Owing to (3.16) and to weak convergence,

$$
\liminf _{h \rightarrow 0} a_{h}\left(u_{h}, u_{h}\right) \geq \liminf _{h \rightarrow 0}\left\|\boldsymbol{\kappa}^{1 / 2} \mathbf{G}_{\omega, h}\left(u_{h}\right)\right\|_{\left[L^{2}(\Omega)\right]^{d}}^{2} \geq\left\|\boldsymbol{\kappa}^{1 / 2} \nabla u\right\|_{\left[L^{2}(\Omega)\right]^{d}}^{2}
$$

Furthermore, still owing to (3.16),

$$
\limsup _{h \rightarrow 0}\left\|\boldsymbol{\kappa}^{1 / 2} \mathbf{G}_{\omega, h}\left(u_{h}\right)\right\|_{\left[L^{2}(\Omega)\right]^{d}}^{2} \leq \limsup _{h \rightarrow 0} a_{h}\left(u_{h}, u_{h}\right)=\limsup _{h \rightarrow 0} \int_{\Omega} f u_{h}=\int_{\Omega} f u=\left\|\boldsymbol{\kappa}^{1 / 2} \nabla u\right\|_{\left[L^{2}(\Omega)\right]^{d}}^{2} .
$$

This classically proves the strong convergence of $\boldsymbol{\kappa}^{1 / 2} \mathbf{G}_{\omega, h}\left(u_{h}\right)$ to $\boldsymbol{\kappa}^{1 / 2} \nabla u$ in $\left[L^{2}(\Omega)\right]^{d}$ and, hence, the strong convergence of $\mathbf{G}_{\omega, h}\left(u_{h}\right)$ to $\nabla u$ in $\left[L^{2}(\Omega)\right]^{d}$. Note that $a_{h}\left(u_{h}, u_{h}\right) \rightarrow\left\|\boldsymbol{\kappa}^{1 / 2} \nabla u\right\|_{\left[L^{2}(\Omega)\right]^{d}}^{2}$ also. Owing to (3.16),

$$
\left(\eta-C_{\mathrm{tr}}^{2} N_{\partial}\right)\left|u_{h}\right|_{\mathrm{J}, \boldsymbol{\kappa}}^{2} \leq a_{h}\left(u_{h}, u_{h}\right)-\left\|\boldsymbol{\kappa}^{1 / 2} \mathbf{G}_{\omega, h}\left(u_{h}\right)\right\|_{\left[L^{2}(\Omega)\right]^{d}}^{2},
$$

and, since $\eta>C_{\mathrm{tr}}^{2} N_{\partial}$ and the right-hand side tends to zero, $\left|u_{h}\right|_{\mathrm{J}, \kappa} \rightarrow 0$. To infer that $\left|u_{h}\right|_{\mathrm{J}} \rightarrow 0$, simply observe that $\left|u_{h}\right|_{\mathrm{J}} \leq \underline{\lambda}^{-1 / 2}\left|u_{h}\right|_{\mathrm{J}, \kappa}$ and that the right-hand side tends to zero.

Remark 3.20 (rough forcing terms). A possible way to handle forcing terms $f$ in $H^{-1}(\Omega)$ consists in replacing the test function by an interpolate in $H_{0}^{1}(\Omega)$ in the second member. For the sake of simplicity, assume that $\mathcal{T}_{h}$ is conforming (if this is not the case, $\mathfrak{S}_{h}$ can be used instead) and let $\mathcal{I}_{\text {Os }}$ denote the Oswald interpolator discussed, e.g., by Burman and Ern [15]. It can be proven that there exists $C$ independent of the meshsize $h$ such that, for all $v_{h} \in V_{h}^{\mathrm{ccg}},\left\|\mathcal{I}_{\mathrm{Os}} v_{h}\right\|_{H^{1}(\Omega)} \leq C_{\text {sta, Os }}\left\|v_{h}\right\| \|$. We consider the following modification of the method (3.6):

$$
\text { Find } u_{h} \in V_{h}^{\text {ccg }} \text { s.t. } a_{h}\left(u_{h}, v_{h}\right)=\left\langle f, \mathcal{I}_{\text {Os }} v_{h}\right\rangle_{-1,1} \text {. }
$$

The a priori estimate for the discrete solutions on the admissible mesh family $\mathcal{T}_{\mathcal{H}}$ is obtained as follows:

$$
C_{\text {sta }} \underline{\lambda}\left\|u_{h}\right\|^{2} \leq a_{h}\left(u_{h}, u_{h}\right)=\left\langle f, \mathcal{I}_{\mathrm{Os}} u_{h}\right\rangle_{-1,1} \leq\|f\|_{H^{-1}(\Omega)}\left\|\mathcal{I}_{\mathrm{Os}} u_{h}\right\|_{H^{1}(\Omega)} \leq C_{\mathrm{sta}, \mathrm{Os}}\|f\|_{H^{-1}(\Omega)}\left\|u_{h}\right\| \|,
$$

hence \|\|$u_{h}\left\|\mid \leq C_{\text {sta, Os }} / C_{\text {sta }}\right\| f \|_{H^{-1}(\Omega)}$. The argument of Theorem 3.19 then applies verbatim.

\section{Steady incompressible Navier-Stokes Equations}

\subsection{The continuous setting}

In the second part of this work we corroborate the claim that ccG methods are easily extended to problems for which a $\mathrm{dG}$ scheme can be devised. Our focus is on the steady incompressible Navier-Stokes (INS) equations 
for $d \in\{2,3\}$,

$$
\begin{aligned}
-\nu \triangle u_{i}+\partial_{j}\left(u_{i} u_{j}\right)+\partial_{i} p & =f_{i} & & \text { in } \Omega, i \in\{1, \ldots, d\}, \\
\partial_{i} u_{i} & =0 & & \text { in } \Omega, \\
u & =0 & & \text { on } \partial \Omega, \\
\langle p\rangle_{\Omega} & =0, & &
\end{aligned}
$$

where the positive real $\nu$ denotes the kinematic viscosity and $f \in\left[L^{2}(\Omega)\right]^{d}$. In (4.1) and throughout this section Einstein's convention on repeated indices is adopted. The natural spaces for the weak formulation of (4.1) are

$$
U:=\left[H_{0}^{1}(\Omega)\right]^{d}, \quad P:=L_{0}^{2}(\Omega), \quad X:=U \times P,
$$

where we have set $L_{0}^{2}(\Omega):=\left\{v \in L^{2}(\Omega) \mid\langle v\rangle_{\Omega}=0\right\}$. We define the bilinear forms $a \in \mathcal{L}(U \times U, \mathbb{R})$ and $b \in \mathcal{L}(U \times P, \mathbb{R})$, and the trilinear form $t \in \mathcal{L}(U \times U \times U, \mathbb{R})$ such that

$$
a(u, v):=\int_{\Omega} \nu \nabla u_{i} \cdot \nabla v_{i}, \quad b(v, q):=-\int_{\Omega} q \nabla \cdot v, \quad t(w, u, v):=\int\left(w \cdot \nabla u_{i}\right) v_{i}-\frac{1}{2} \int_{\Omega}(\nabla \cdot w)(u \cdot v) .
$$

The trilinear form $t$ includes Temam's device [43] to control the kinetic energy balance as this is needed in what follows for the asymptotic consistency of its discrete counterpart. The weak formulation of system (4.1) is:

$$
\text { Find }(u, p) \in X \text { s.t. } c((u, p),(v, q))+t(u, u, v)=\int_{\Omega} f \cdot v \text { for all }(v, q) \in X,
$$

with bilinear form $c \in \mathcal{L}(X \times X, \mathbb{R})$ such that $c((u, p),(v, q))=a(u, v)+b(v, p)-b(u, q)$.

\subsection{The discrete setting}

We seek a discretization of (4.2) based on the following discrete spaces:

$$
U_{h}:=\left[V_{h}^{\mathrm{ccg}}\right]^{d}, \quad P_{h}:=\mathbb{P}_{d}^{0}\left(\mathcal{T}_{h}\right) / \mathbb{R}, \quad X_{h}:=U_{h} \times P_{h} .
$$

The main difficulties in the approximation of the INS equations lie in the discretization of the velocity-pressure coupling and of the convective term. In our case, the velocity-pressure coupling is stabilized by penalizing the pressure jumps across interfaces with a weight proportional to the meshsize; see, e.g., [19]. As regards the convective term, we use the non-dissipative trilinear form recently proposed by Di Pietro and Ern [22], which has proven suitable to convection-dominated regimes; see also Botti and Di Pietro [9] for the application to a $\mathrm{dG}$ discretization of the advection step in the context of a pressure-correction time-integration scheme. Since the convergence analysis is similar as for the dG method of [22], the proofs of the results that hold a fortiori are sometimes omitted to leave room to specific issues related to the ccG method.

\subsubsection{Velocity-pressure coupling}

The velocity-pressure coupling is based on the bilinear form $b_{h} \in \mathcal{L}\left(U_{h} \times P_{h}, \mathbb{R}\right)$ such that

$$
b_{h}\left(v_{h}, q_{h}\right):=-\int_{\Omega} q_{h} \nabla_{h} \cdot v_{h}+\sum_{F \in \mathcal{F}_{h}} \int_{F} \llbracket v_{h} \rrbracket \cdot \mathbf{n}_{F}\left\{q_{h}\right\}=-\sum_{F \in \mathcal{F}_{h}^{\mathrm{i}}} \int_{F}\left\{v_{h}\right\} \cdot \mathbf{n}_{F} \llbracket q_{h} \rrbracket .
$$

A useful equivalent form for $b_{h}$ can be inferred introducing the discrete divergence operator $\mathrm{D}_{h}:\left[H^{1}\left(\mathcal{T}_{h}\right)\right]^{d} \rightarrow$ $\mathbb{P}_{d}^{0}\left(\mathcal{T}_{h}\right)$ defined as follows: For all $v \in\left[H^{1}\left(\mathcal{T}_{h}\right)\right]^{d}$,

$$
\mathrm{D}_{h}(v):=\mathbf{G}_{h}\left(v_{i}\right) \cdot \mathbf{e}_{i} .
$$


The subscript $\omega$ has been omitted from discrete gradient since $\boldsymbol{\kappa}=\nu \mathbf{1}_{d}$ (as the kinematic viscosity is homogeneous and isotropic) implies $\omega_{T_{1}, F}=\omega_{T_{2}, F}=1 / 2$ for all $F \in \mathcal{F}_{h}^{\mathrm{i}}$ with $F \subset \partial T_{1} \cap \partial T_{2}$. The discrete divergence $\mathrm{D}_{h}$ is defined as the trace of $\mathbf{G}_{h}$ applied to a vector function. It follows from (4.3) that

$$
\forall\left(v_{h}, q_{h}\right) \in X_{h}, \quad b_{h}\left(v_{h}, q_{h}\right)=-\int_{\Omega} q_{h} \mathrm{D}_{h}\left(v_{h}\right) .
$$

We let, for all vector functions $v_{h} \in U_{h}$,

$$
\left\|v_{h}\right\|\left\|^{2}:=\sum_{i=1}^{d}\right\| v_{h, i} \|^{2}, \quad\left|v_{h}\right|_{\mathrm{J}}^{2}:=\sum_{i=1}^{d}\left|v_{h, i}\right|_{\mathrm{J}}^{2} .
$$

As the discrete operator associated to the discrete bilinear form $b_{h}$ is not surjective, pressure stabilization must be introduced. To this end, we define the bilinear form $s_{h} \in \mathcal{L}\left(P_{h} \times P_{h}, \mathbb{R}\right)$ and the associated seminorm $|\cdot|_{p}$ such that

$$
s_{h}\left(p_{h}, q_{h}\right):=\sum_{F \in \mathcal{F}_{h}^{\mathrm{i}}} h_{F} \int_{F} \llbracket p_{h} \rrbracket \llbracket q_{h} \rrbracket, \quad\left|q_{h}\right|_{p}^{2}:=s_{h}\left(q_{h}, q_{h}\right) .
$$

We are now ready to state the main result of this section.

Lemma 4.1 (stability of the velocity-pressure coupling). Under Assumption 3.7, there exists $\beta>0$ independent of the meshsize $h$ such that

$$
\forall q_{h} \in P_{h}, \quad \beta\left\|q_{h}\right\|_{L^{2}(\Omega)} \leq \sup _{w_{h} \in U_{h} \backslash\{0\}} \frac{b_{h}\left(w_{h}, q_{h}\right)}{\left\|w_{h}\right\|}+\left|q_{h}\right|_{p} .
$$

Proof. In the proof we abbreviate $a \lesssim b$ the inequality $a \leq C b$ where $C$ can depend on the mesh regularity parameters and on $\Omega$ but not on the meshsize $h$. Owing to the surjectivity of the divergence operator from $U$ to $P$, there exists $C_{\Omega}>0$ uniquely depending on the domain $\Omega$ such that, for all $q \in L_{0}^{2}(\Omega)$, there exists $v \in\left[H_{0}^{1}(\Omega)\right]^{d}$ such that

$$
\nabla \cdot v=q, \quad C_{\Omega}\|v\|_{\left[H^{1}(\Omega)\right]^{d}} \leq\|q\|_{L^{2}(\Omega)} .
$$

Let now $q=q_{h}$, denote by $v$ the element of $\left[H_{0}^{1}(\Omega)\right]^{d}$ satisfying (4.5) and set $\mathbf{v}_{h}:=\left(\langle v\rangle_{T}\right)_{T \in \mathcal{T}_{h}} \in \mathbb{V}_{h}$ and $v_{h}:=\mathfrak{R}_{h}^{\mathrm{ccg}}\left(\mathbf{v}_{h}\right) \in V_{h}^{\mathrm{ccg}}$. Then,

$$
\begin{aligned}
C_{\Omega}\|v\|_{\left[H^{1}(\Omega)\right] d}\left\|q_{h}\right\|_{L^{2}(\Omega)} & \leq\left\|q_{h}\right\|_{L^{2}(\Omega)}^{2}=\int_{\Omega} q_{h} \nabla \cdot v=\sum_{F \in \mathcal{F}_{h}^{\mathrm{i}}} \int_{F} \llbracket q_{h} \rrbracket\{v\} \cdot \mathbf{n}_{F}=-b_{h}\left(v_{h}, q_{h}\right)+\sum_{F \in \mathcal{F}_{h}^{\mathrm{i}}} \int_{F} \llbracket q_{h} \rrbracket\left\{v-v_{h}\right\} \cdot \mathbf{n}_{F} \\
& \leq\left.\left\{\sup _{w_{h} \in U_{h} \backslash\{0\}} \frac{b_{h}\left(w_{h}, q_{h}\right)}{\left\|w_{h}\right\| \mid}\right\}\left\|v_{h}\right\||+| q_{h}\right|_{p} \times\left\{\sum_{F \in \mathcal{F}_{h}^{\mathrm{i}}} h_{F}^{-1} \int_{F}\left|\left\{v-v_{h}\right\}\right|^{2}\right\}^{1 / 2} .
\end{aligned}
$$

It follows from Lemma 4.2 that $\left\|v_{h}\right\|\|\lesssim\| v \|_{\left[H^{1}(\Omega)\right]^{d}}$. Moreover, using the continuous trace inequality (2.5) together with the approximation properties of $\mathcal{T}_{\mathcal{H}}$, it is readily proven that $\sum_{F \in \mathcal{F}_{h}^{\mathrm{i}}} h_{F}^{-1} \int_{F}\left|\left\{v-v_{h}\right\}\right|^{2} \lesssim\|v\|_{H^{1}(\Omega)}^{2}$.

The following lemma establishes the $H^{1}$-stability property used in the proof of Lemma 4.1. Observe that, unlike elsewhere, the degrees of freedom are here interpreted as average values over the cells, since the regularity of the function $v$ is in general insufficient for point values to be defined inside elements. The proof is given in Section A.3. 
Lemma $4.2\left(H^{1}\right.$-stability). Under Assumption 3.7, for all $v \in H_{0}^{1}(\Omega)$ there holds

$$
\left\|v_{h}\right\| \leq \leq\|v\|_{H^{1}(\Omega)},
$$

where $\mathbf{v}_{h}=\left(\langle v\rangle_{T}\right)_{T \in \mathcal{T}_{h}} \in \mathbb{V}_{h}, v_{h}=\mathfrak{R}_{h}^{\mathrm{ccg}}\left(\mathbf{v}_{h}\right) \in V_{h}^{\mathrm{ccg}}$ and $C$ is independent of the meshsize $h$.

4.2.2. A non-dissipative convective trilinear form

The discrete convective trilinear form $t_{h} \in \mathcal{L}\left(U_{h} \times U_{h} \times U_{h}, \mathbb{R}\right)$ is given by

$$
\begin{aligned}
t_{h}\left(w_{h}, u_{h}, v_{h}\right):= & \int_{\Omega}\left(w_{h} \cdot \nabla_{h} u_{h, i}\right) v_{h, i}-\sum_{F \in \mathcal{F}_{h}^{\mathrm{i}}} \int_{F}\left\{w_{h}\right\} \cdot \mathbf{n}_{F} \llbracket u_{h} \rrbracket \cdot\left\{v_{h}\right\} \\
& +\frac{1}{2} \int_{\Omega}\left(\nabla_{h} \cdot w_{h}\right)\left(u_{h} \cdot v_{h}\right)-\frac{1}{2} \sum_{F \in \mathcal{F}_{h}} \int_{F} \llbracket w_{h} \rrbracket \cdot \mathbf{n}_{F}\left\{u_{h} \cdot v_{h}\right\} .
\end{aligned}
$$

The following lemma collects some important results. The proof essentially follows [22], Proposition 5.2. In particular, the Sobolev embedding (2.15) for $q=4$ is required, which limits the space dimension to $d \in\{2,3\}$.

Lemma 4.3 (properties of the trilinear form $t_{h}$ ). For all $h \in \mathcal{H}$, let $\mathcal{U}_{h}:=\left[\mathbb{P}_{d}^{k}\left(\mathcal{T}_{h}\right)\right]^{d}$ for some $k>0$. The trilinear form $t_{h}$ defined by (4.6) enjoys the following properties:

(i) Non-dissipativity. For all $w_{h}, v_{h} \in \mathcal{U}_{h}, t_{h}\left(w_{h}, v_{h}, v_{h}\right)=0$.

(ii) Boundedness. There is $C_{\mathrm{bnd}, t}$ independent of the meshsize $h$ such that, for all $w_{h}, u_{h}, v_{h} \in \mathcal{U}_{h}$, $t_{h}\left(w_{h}, u_{h}, v_{h}\right) \leq C_{\mathrm{bnd}, t}\left\|w_{h}\right\|\left|\left\||| u_{h}|||||| v_{h} \mid\right\|\right.$.

(iii) Asymptotic consistency for smooth functions. Let $\left(v_{h}\right)_{h \in \mathcal{H}}$ be a sequence in $\left(\mathcal{U}_{h}\right)_{h \in \mathcal{H}}$ bounded in the $\||\cdot|\|-$ norm. Then, for all $\Phi \in\left[C_{0}^{\infty}(\Omega)\right]^{d}$, as $h \rightarrow 0$, up to a subsequence, $t_{h}\left(v_{h}, v_{h}, \Phi_{h}\right) \rightarrow t(v, v, \Phi)$, where $\Phi_{h}:=\left(\mathfrak{R}_{h}^{\mathrm{ccg}} \circ \mathcal{I}_{h}^{\mathrm{ccg}}\right)(\Phi) \in U_{h}$ and $v \in U$ is the limit provided by Theorem 2.15 .

(iv) Asymptotic consistency for test functions. In the setting of point (iii), further suppose that $\mathbf{G}_{h}\left(v_{h}\right) \rightarrow \nabla v$ strongly in $\left[L^{2}(\Omega)\right]^{d}$ and that $\left|v_{h}\right|_{J} \rightarrow 0$. Let $\left(w_{h}\right)_{h \in \mathcal{H}}$ be another sequence in $\left(\mathcal{U}_{h}\right)_{h \in \mathcal{H}}$ bounded in the $\| \cdot|| \mid-$-norm. Then, as $h \rightarrow 0$, up to a subsequence, $t_{h}\left(v_{h}, v_{h}, w_{h}\right) \rightarrow t(v, v, w)$, with both $v, w \in U$ resulting from Theorem 2.15 .

The discrete problem reads

$$
\text { Find }\left(u_{h}, p_{h}\right) \in X_{h} \text { s.t. } c_{h}\left(\left(u_{h}, p_{h}\right),\left(v_{h}, q_{h}\right)\right)+t_{h}\left(u_{h}, u_{h}, v_{h}\right)=\int_{\Omega} f \cdot v_{h} \text { for all }\left(v_{h}, q_{h}\right) \in X_{h} \text {, }
$$

with bilinear form $c_{h} \in \mathcal{L}\left(X_{h} \times X_{h}, \mathbb{R}\right)$ such that $c_{h}:=\sum_{i=1}^{d} a_{h}\left(u_{h, i}, v_{h, i}\right)+b_{h}\left(v_{h}, p_{h}\right)-b_{h}\left(u_{h}, q_{h}\right)+s_{h}\left(p_{h}, q_{h}\right)$ and we have set $\boldsymbol{\kappa}=\nu \mathbf{1}_{d}$ in the expression of $a_{h}$.

\subsection{Convergence}

We study the convergence of the method (4.7) in the spirit of Section 3.4. As the ccG space $V_{h}^{\text {ccg }}$ is a subspace of $\mathbb{P}_{d}^{1}\left(\mathcal{T}_{h}\right)$, some of the results presented in [22], Section 5, in the context of dG methods hold a fortiori. In such cases, the details of the proofs are omitted in order to restrict the focus to the peculiarities of the proposed ccG method. Also, since the diffusion coefficient is homogeneous, the standard test space $C_{0}^{\infty}(\Omega)$ can replace $\mathcal{Q}_{P_{\Omega}, \kappa}$ in the convergence proof. The following lemmata contain results that are instrumental to the analysis.

Lemma 4.4 (properties of $\mathrm{D}_{h}$ ). The discrete divergence $\mathrm{D}_{h}$ enjoys the following properties:

(i) Consistency for smooth functions. Let $\Phi \in\left[C_{0}^{\infty}(\Omega)\right]^{d}$ and set $\Phi_{h}:=\left(\mathfrak{R}_{h}^{\mathrm{ccg}} \circ \mathcal{I}_{h}^{\mathrm{ccg}}\right)(\Phi) \in U_{h}$. Then, under Assumption 3.7 , as $h \rightarrow 0, \mathrm{D}_{h}\left(\Phi_{h}\right) \rightarrow \nabla \cdot \Phi$ strongly in $L^{2}(\Omega)$.

(ii) Weak asymptotic consistency for test functions. Let $\left(v_{h}\right)_{h \in \mathcal{H}}$ be a sequence in $U_{h}$ uniformly bounded in the $|\|\cdot\|| \|$ norm. The, as $h \rightarrow 0$, up to a subsequence, $\mathrm{D}_{h}\left(v_{h}\right) \rightarrow \nabla \cdot v$ weakly in $L^{2}(\Omega)$, where $v \in\left[H_{0}^{1}(\Omega)\right]^{d}$ is the limit resulting from Theorem 2.15 . 
Proof. Point (i) is a direct consequence of Theorem 3.8 together with the fact that $\boldsymbol{\kappa}=\nu \mathbf{1}_{d}$ implies $C_{0}^{\infty}(\Omega) \subset$ $\mathcal{Q}_{P_{\Omega}, \kappa}$ and that $\llbracket \Phi \rrbracket_{F}(\mathbf{x})=0$ for all $F \in \mathcal{F}_{h}$ and all $\mathbf{x} \in F$. Point (ii) immediately follows from Lemma 2.16 .

Lemma 4.5 (existence of a solution to (4.7)). There exists $\left(u_{h}, p_{h}\right) \in X_{h}$ solution to (4.7).

Proof. The proof is based on a topological degree argument and it follows [22], Proposition 5.1. The use of a topological degree argument to assert the existence of a discrete solution can be traced back to Eymard et al. [34].

The first step in the convergence proof is to derive a uniform a priori estimate on a suitable norm of the discrete solution. This estimate is used to infer a compactness property for the sequence of discrete solutions. To this end, we introduce the following norm on $X_{h}$ :

$$
\left\|\left(v_{h}, q_{h}\right)\right\|_{\mathrm{ns}}^{2}:=\left\|\left.\left|v_{h}\left\|^{2}+\right\| q_{h} \|_{L^{2}(\Omega)}^{2}+\right| q_{h}\right|_{p} ^{2} .\right.
$$

Lemma 4.6 (a priori estimate). There exists $\gamma>0$ independent of the meshsize $h$ such that

$$
\gamma \mid\left\|\left(u_{h}, p_{h}\right)\right\|\left\|_{\mathrm{ns}} \leq \sigma_{2}\right\| f \|_{\left[L^{2}(\Omega)\right]^{d}}+C_{\mathrm{bnd}, t}\left(\nu C_{\mathrm{sta}}\right)^{-1}\left(\sigma_{2}\|f\|_{\left[L^{2}(\Omega)\right]^{d}}\right)^{2}
$$

where $C_{\mathrm{bnd}, t}$ results from Lemma 4.3ii, $C_{\mathrm{sta}}$ is the coercivity constant of $a_{h}$, and $\sigma_{2}$ results from Theorem 2.14.

Proof. The proof proceeds along the lines of [22], Lemma 5.1. The details are omitted for the sake of brevity.

Theorem 4.7 (convergence). Let $\left(\left(u_{h}, p_{h}\right)\right)_{h \in \mathcal{H}}$ be a sequence of approximate solutions generated by solving the discrete problems (4.7) on the admissible mesh sequence $\mathcal{T}_{\mathcal{H}}$. Then, under Assumption 3.7 , as $h \rightarrow 0$, up to a subsequence, (i) $u_{h} \rightarrow u$ strongly in $\left[L^{2}(\Omega)\right]^{d}$, (ii) $\nabla_{h} u_{h} \rightarrow \nabla u$ strongly in $\left[L^{2}(\Omega)\right]^{d, d}$, (iii) $\left|u_{h}\right|_{\mathrm{J}} \rightarrow 0$, (iv) $p_{h} \rightarrow p$ strongly in $L^{2}(\Omega)$, (v) $\left|p_{h}\right|_{p} \rightarrow 0$, with $(u, p) \in X$ solution to (4.2). If the continuous solution (u,p) is unique, the convergence property extends to the whole sequence.

Proof. (i) Compactness. Owing to the a priori estimate (4.8), by Theorem 2.15 together with Lemma 2.16 there exists $(\bar{u}, \bar{p}) \in X$ such that, up to a subsequence, $u_{h} \rightarrow \bar{u}$ strongly in $\left[L^{2}(\Omega)\right]^{d}, \mathbf{G}_{h}\left(u_{h, i}\right) \rightarrow \nabla \bar{u}_{i}$ weakly in $\left[L^{2}(\Omega)\right]^{d}$ for $i \in\{1, \ldots, d\}$, and $p_{h} \rightarrow \bar{p}$ weakly in $L^{2}(\Omega)$ (as the sequence $\left(p_{h}\right)_{h \in \mathcal{H}}$ is bounded in the $L^{2}$ norm uniformly with respect to $h$ ). For the sake of conciseness, subsequences are not renumbered in what follows. (ii) Identification of the limit and convergence of a subsequence. Let $\Phi \in\left[C_{0}^{\infty}(\Omega)\right]^{d}$ and set $\Phi_{h}:=\left(\Re_{h}^{\text {ccg }} \circ \mathcal{I}_{h}^{\text {ccg }}\right)(\Phi) \in U_{h}$. Using $\left(\Phi_{h}, 0\right)$ as a test function yields

$$
a_{h}\left(u_{h}, \Phi_{h}\right)+b_{h}\left(\Phi_{h}, p_{h}\right)+t_{h}\left(u_{h}, u_{h}, \Phi_{h}\right)=\int_{\Omega} f \cdot \Phi_{h} .
$$

Clearly, as $h \rightarrow 0$, the right-hand side tends to $\int_{\Omega} f \cdot \Phi$. Furthermore, by virtue of Lemma 3.17 , the first term in the left-hand side converges to $a(u, \Phi)=\int_{\Omega} \nu \nabla u_{i} \cdot \nabla \Phi_{i}$. Using (4.4), the second term can be written as $b_{h}\left(\Phi_{h}, p_{h}\right)=-\int_{\Omega} p_{h} \mathrm{D}_{h}\left(\Phi_{h}\right)$. Owing to the weak convergence of $\left(p_{h}\right)_{h \in \mathcal{H}}$ to $\bar{p}$ in $L^{2}(\Omega)$ and to the strong convergence of $\left(\mathrm{D}_{h}\left(\Phi_{h}\right)\right)_{h \in \mathcal{H}}$ to $\nabla \cdot \Phi$ in $L^{2}(\Omega)$ stated in Lemma 4.4, this term converges to $b(\Phi, \bar{p})=-\int_{\Omega} \bar{p} \nabla \cdot \Phi$. Finally $t_{h}\left(u_{h}, u_{h}, \Phi_{h}\right)$ tends to $t(\bar{u}, \bar{u}, \Phi)$ owing to Lemma 4.3iii. As a result,

$$
a(\bar{u}, \Phi)+b(\Phi, \bar{p})+t(\bar{u}, \bar{u}, \Phi)=\int_{\Omega} f \cdot \Phi .
$$

Let now $\varphi \in C_{0}^{\infty}(\Omega) / \mathbb{R}$ and set $\varphi_{h}:=\pi_{h}^{0} \varphi$, where $\pi_{h}^{0}$ denotes the $L^{2}$-orthogonal projection onto $\mathbb{P}_{d}^{0}\left(\mathcal{T}_{h}\right)$. Using $\left(0, \varphi_{h}\right)$ as a test function yields

$$
-b_{h}\left(u_{h}, \varphi_{h}\right)+s_{h}\left(p_{h}, \varphi_{h}\right)=0 .
$$

Clearly, $-b_{h}\left(u_{h}, \varphi_{h}\right)=\int_{\Omega} \varphi_{h} \mathrm{D}_{h}\left(u_{h}\right)$ tends to $\int_{\Omega} \varphi \nabla \cdot \bar{u}$ as $h \rightarrow 0$ since $\left(\mathrm{D}_{h}\left(u_{h}\right)\right)_{h \in \mathcal{H}}$ weakly converges to $\nabla \cdot \bar{u}$ in $L^{2}(\Omega)$ owing to Lemma 4.4ii and $\left(\varphi_{h}\right)_{h \in \mathcal{H}}$ strongly converges to $\varphi$ in $L^{2}(\Omega)$. Furthermore, using the a priori 
estimate (4.8), $\left|s_{h}\left(p_{h}, \varphi_{h}\right)\right| \leq\left|p_{h}\right|_{p}\left|\varphi_{h}\right|_{p} \leq C\left|\varphi_{h}\right|_{p}$ with $C$ independent of the meshsize $h$ and this upper bound tends to zero. Hence,

$$
\int_{\Omega} \varphi \nabla \cdot \bar{u}=0
$$

By density of $\left[C_{0}^{\infty}(\Omega)\right]^{d} \times\left(C_{0}^{\infty}(\Omega) / \mathbb{R}\right)$ in $X$, this shows that $(\bar{u}, \bar{p})=(u, p)$ solution to $(4.2)$.

(iii) Strong convergence of the velocity gradient and of the velocity and pressure jumps. Owing to the nondissipativity of $t_{h}$, and recalling (3.14)

$$
\int_{\Omega} f \cdot u_{h}=c_{h}\left(\left(u_{h}, p_{h}\right),\left(u_{h}, p_{h}\right)\right)=a_{h}\left(u_{h}, u_{h}\right)+s_{h}\left(p_{h}, p_{h}\right) \geq a_{h}\left(u_{h}, u_{h}\right) \geq \nu \sum_{i=1}^{d}\left\|\mathbf{G}_{h}\left(u_{h, i}\right)\right\|_{\left[L^{2}(\Omega)\right]^{d}}^{2} .
$$

Thus, $\nu \lim \sup _{h \rightarrow 0} \sum_{i=1}^{d}\left\|\mathbf{G}_{h}\left(u_{h, i}\right)\right\|_{\left[L^{2}(\Omega)\right]^{d, d}}^{2} \leq \limsup _{h \rightarrow 0} \int_{\Omega} f \cdot u_{h}=\int_{\Omega} f \cdot u=\nu\|\nabla u\|_{\left[L^{2}(\Omega)\right]^{d, d}}^{2}$. Proceeding as in point (iv) of Theorem 3.19, it is then inferred that $\mathbf{G}_{h}\left(u_{h, i}\right) \rightarrow \nabla u_{i}$ in $\left[L^{2}(\Omega)\right]^{d}$ for all $i \in\{1, \ldots, d\}$ and that $\left|u_{h}\right|_{\mathrm{J}} \rightarrow 0$. Finally, since $\left|p_{h}\right|_{p}^{2}=b_{h}\left(u_{h}, p_{h}\right)=\int_{\Omega} f \cdot u_{h}-a_{h}\left(u_{h}, u_{h}\right)$, we conclude that $\left|p_{h}\right|_{p} \rightarrow 0$.

(v) Strong convergence of the pressure. Let $v \in\left[H_{0}^{1}(\Omega)\right]^{d}$ be such that $\nabla \cdot v=p_{h}$ with $\|v\|_{\left[H^{1}(\Omega)\right]^{d}} \leq C_{\Omega}\left\|p_{h}\right\|_{L^{2}(\Omega)}$, and set $v_{h}:=\mathfrak{R}_{h}^{\mathrm{ccg}}\left(\mathbf{v}_{h}\right) \in V_{h}^{\mathrm{ccg}}$ with $\mathbf{v}_{h}=\left(\langle v\rangle_{T}\right)_{T \in \mathcal{T}_{h}} \in \mathbb{V}_{h}$. In the rest of the proof we abbreviate $a \lesssim b$ the inequality $a \leq C b$ with $C$ independent of the meshsize $h$. Proceeding as in the proof of Lemma 4.1 yields

$$
\left\|p_{h}\right\|_{L^{2}(\Omega)}^{2} \lesssim\left|p_{h}\right|_{p}\left\|p_{h}\right\|_{L^{2}(\Omega)}-b_{h}\left(v_{h}, p_{h}\right) \lesssim\left|p_{h}\right|_{p}\left\|p_{h}\right\|_{L^{2}(\Omega)}+a_{h}\left(u_{h}, v_{h}\right)+t_{h}\left(u_{h}, u_{h}, v_{h}\right)-\int_{\Omega} f \cdot v_{h} .
$$

Let $\mathfrak{T}_{i}, i \in\{1, \ldots, 4\}$ denote the terms in the right-hand side. Since $\left|p_{h}\right|_{p}$ tends to zero and $\left\|p_{h}\right\|_{L^{2}(\Omega)}$ is bounded, $\mathfrak{T}_{1}$ converges to zero. Furthermore, since the sequence $\left(v_{h}\right)_{h \in \mathcal{H}}$ is bounded in the $|\|\cdot \mid\|$-norm because $\left\|v_{h}\right\|\|\| v\left\|_{\left[H^{1}(\Omega)\right]^{d}} \lesssim\right\| p_{h} \|_{L^{2}(\Omega)}$ there is $\bar{v} \in\left[H_{0}^{1}(\Omega)\right]^{d}$ such that, up to a subsequence, $v_{h} \rightarrow \bar{v}$ strongly in $\left[L^{2}(\Omega)\right]^{d}$ and $\mathbf{G}_{h}\left(v_{h, i}\right) \rightarrow \nabla \bar{v}_{i}$ weakly in $\left[L^{2}(\Omega)\right]^{d}$ for $i \in\{1, \ldots, d\}$. Owing to the uniqueness of the limit in the distribution sense, it is inferred that $\nabla \cdot \bar{v}=p$. There holds

$$
\mathfrak{T}_{2}=a_{h}\left(u_{h}, v_{h}\right)=\int_{\Omega} \nu \mathbf{G}_{h}\left(u_{h, i}\right) \cdot \mathbf{G}_{h}\left(v_{h, i}\right)+s_{h}\left(u_{h}, v_{h}\right)=\mathfrak{T}_{2,1}+\mathfrak{T}_{2,2}
$$

Owing to the strong convergence of $\left(\mathbf{G}_{h}\left(u_{h, i}\right)\right)_{h \in \mathcal{H}}$ in $\left[L^{2}(\Omega)\right]^{d}$ proven in the previous point together with the weak convergence of $\left(\mathbf{G}_{h}\left(v_{h, i}\right)\right)_{h \in \mathcal{H}}$ in $\left[L^{2}(\Omega)\right]^{d}$ yields $\mathfrak{T}_{2,1} \rightarrow \int_{\Omega} \nabla u_{i} \cdot \nabla \bar{v}_{i}$. Moreover, $\left|\mathfrak{T}_{2,2}\right| \lesssim\left|u_{h}\right|_{\mathrm{J}}\left|v_{h}\right|_{\mathrm{J}}$, which converges to zero. Owing to Lemma 4.3iv, $\mathfrak{T}_{3} \rightarrow t(u, u, \bar{v})$. Finally, since $\mathfrak{T}_{4} \rightarrow \int_{\Omega} f \cdot \bar{v}$, the strong convergence of the pressure in $L^{2}$ classically follows from

$$
\limsup _{h \rightarrow 0}\left\|p_{h}\right\|_{L^{2}(\Omega)}^{2} \leq \int_{\Omega} \nabla u_{i} \cdot \nabla \bar{v}_{i}+t(u, u, \bar{v})-\int_{\Omega} f \cdot \bar{v}=\int_{\Omega} p \nabla \cdot \bar{v}=\|p\|_{L^{2}(\Omega)}^{2} .
$$

\section{Numerical EXAMPLES}

\subsection{Pure diffusion}

Anisotropy. To investigate the behaviour of the method (3.6) for anisotropic diffusion tensors we consider the following exact solution to problem (3.2) in $d=2$ :

$$
u=\sin (\pi x) \sin (\pi y), \quad \kappa=\left[\begin{array}{ll}
1 & 0 \\
0 & \epsilon
\end{array}\right], \quad f=(1+\epsilon) \pi^{2} \sin (\pi x) \sin (\pi y),
$$

with anisotropy ratio $\epsilon=10^{-3}$. The discrete problem is solved on the Kershaw mesh sequence 4.2 of the FVCA5 benchmark [37] ( $c f$. Fig. 3a), and the results are listed in Table 1 . Besides the errors in the $L^{2}-$ and 
TABLE 1. Anisotropic test case.

\begin{tabular}{ccccccc}
\hline $\operatorname{card}\left(\mathcal{T}_{h}\right)$ & $\left\|u-u_{h}\right\|_{L^{2}(\Omega)}$ & Order & $\left\|u-u_{h}\right\|$ & Order & $\max _{F \in \mathcal{F}_{h}^{\text {i }}}\left\|\mathbf{A}_{\mathfrak{g}_{F}}^{-1}\right\|_{\infty}$ & cg+AMG it. \\
\hline 9801 & $1.2396 \mathrm{e}-02$ & - & $5.1296 \mathrm{e}-02$ & - & $1.0028 \mathrm{e}+03$ & 41 \\
17424 & $6.8589 \mathrm{e}-03$ & 2.06 & $3.3572 \mathrm{e}-02$ & 1.47 & $1.0018 \mathrm{e}+03$ & 49 \\
27225 & $3.9340 \mathrm{e}-03$ & 2.49 & $2.3897 \mathrm{e}-02$ & 1.52 & $1.0013 \mathrm{e}+03$ & 55 \\
39204 & $2.5485 \mathrm{e}-03$ & 2.38 & $1.8058 \mathrm{e}-02$ & 1.54 & $1.0009 \mathrm{e}+03$ & 62 \\
\hline
\end{tabular}

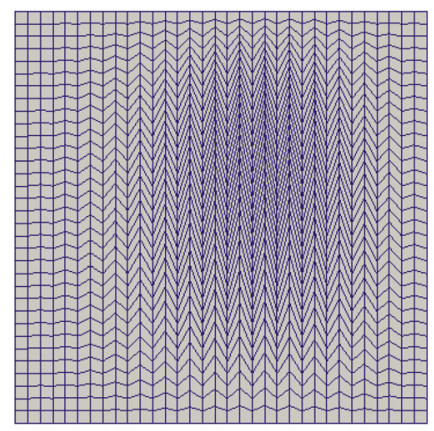

(a) Example of mesh for the test case $(43)$

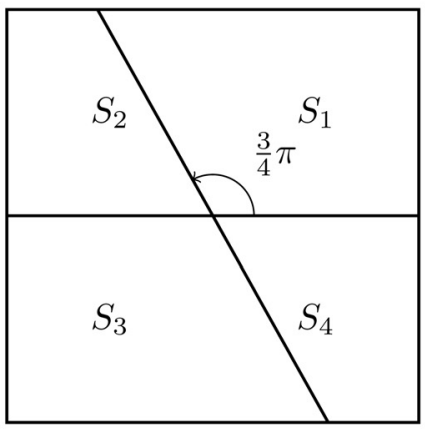

(b) Partition $P_{\Omega}$ for the test cases $(44)-(46)$

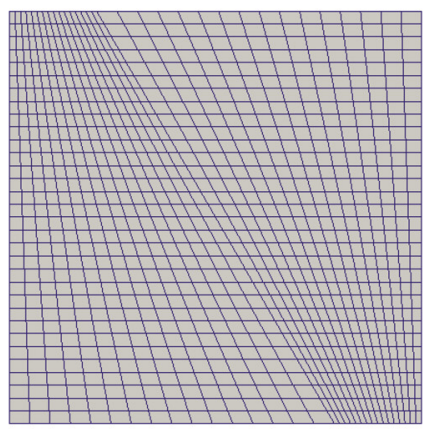

(c) Example of mesh for the test cases $(44)-(46)$

Figure 3. Configurations for pure diffusion test cases.

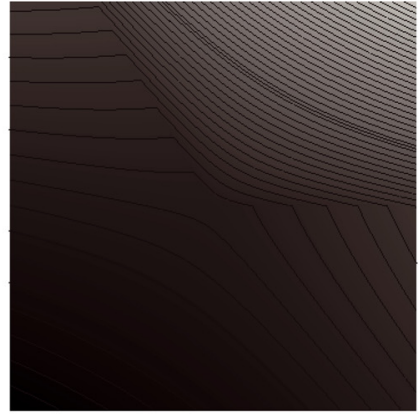

(a) Test case (44), $u \in H^{2.29}(\Omega)$

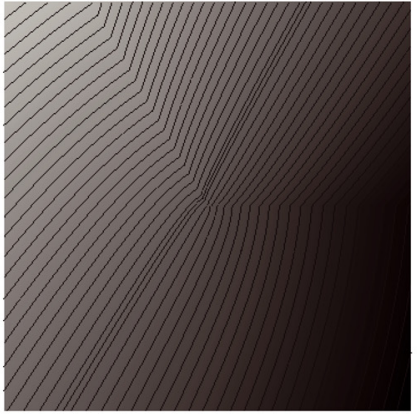

(b) Test case (45), $u \in H^{1.79}(\Omega)$

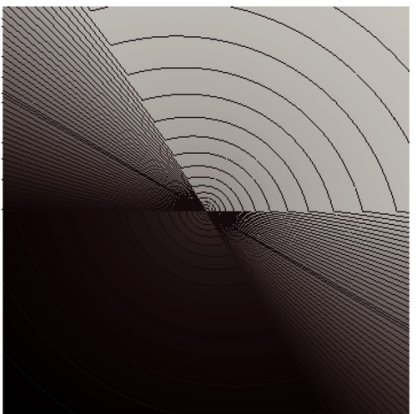

(c) Test case (46), $u \in H^{1.29}(\Omega)$

FiguRE 4. Numerical solutions of problem $(3.1)(128 \times 128$ mesh $)$.

energy-norms, Table 1 lists the maximum $\|\cdot\|_{\infty}$-norm of the matrices defined by (2.11) as well as the number of conjugate gradient iterations with AMG preconditioner required to solve the linear system. An inspection of column 6 shows that Assumption 3.7 is satisfied since the largest norm of the matrices involved in the local construction remains almost constant when refining the mesh. Here and in what follows, we estimate the order of convergence as

$$
\text { order }=d \ln \left(e_{1} / e_{2}\right) / \ln \left(\operatorname{card}\left(\mathcal{T}_{h_{2}}\right) / \operatorname{card}\left(\mathcal{T}_{h_{1}}\right)\right) \text {, }
$$

where $e_{1}$ and $e_{2}$ denote, respectively, the discretization errors on $\mathcal{T}_{h_{1}}$ and $\mathcal{T}_{h_{2}}, h_{1}, h_{2} \in \mathcal{H}$.

Heterogeneity. To assess the behaviour of the method with respect to the heterogeneity of the diffusion tensor we consider the solutions proposed in [3], Section III. The domain $\Omega=(0,1)^{2}$ is partitioned into four areas corresponding to different values of the diffusion coefficient $\boldsymbol{\kappa}$ as depicted in Figure $3 \mathrm{~b}$, and we consider a sequence of meshes matching Assumption 2.9; see Figure 3c. The permeability coefficient is such that $\boldsymbol{\kappa}_{\mid S_{1}}=k_{1} \mathbf{1}_{d}$ and 
TABLE 2. Heterogeneous solution (5.2), $u \in H^{2.29}(\Omega)$.

\begin{tabular}{ccccccc}
\hline $\operatorname{card}\left(\mathcal{T}_{h}\right)$ & $\left\|u-u_{h}\right\|_{L^{2}(\Omega)}$ & Order & $\left\|u-u_{h}\right\|$ & Order & $\max _{F \in \mathcal{F}_{h}^{\mathrm{i}}}\left\|\mathbf{A}_{\mathfrak{g}_{F}}^{-1}\right\|_{\infty}$ & cg+AMG it. \\
\hline $8 \times 8$ & $6.5808 \mathrm{e}-3$ & - & $1.9547 \mathrm{e}-2$ & - & $1.1656 \mathrm{e}+1$ & 5 \\
$16 \times 16$ & $1.9161 \mathrm{e}-3$ & 1.78 & $7.8171 \mathrm{e}-3$ & 1.33 & $1.2094 \mathrm{e}+1$ & 6 \\
$32 \times 32$ & $4.3365 \mathrm{e}-3$ & 2.14 & $3.2200 \mathrm{e}-3$ & 1.28 & $1.2301 \mathrm{e}+1$ & 7 \\
$64 \times 64$ & $9.6024 \mathrm{e}-5$ & 2.18 & $1.3410 \mathrm{e}-3$ & 1.26 & $1.2401 \mathrm{e}+1$ & 8 \\
$128 \times 128$ & $2.1670 \mathrm{e}-5$ & 2.15 & $5.5576 \mathrm{e}-4$ & 1.27 & $1.2451 \mathrm{e}+1$ & 9 \\
\hline
\end{tabular}

TABLE 3. Heterogeneous solution (5.3), $u \in H^{1.79}(\Omega)$.

\begin{tabular}{ccccccc}
\hline $\operatorname{card}\left(\mathcal{T}_{h}\right)$ & $\left\|u-u_{h}\right\|_{L^{2}(\Omega)}$ & Order & $\left\|u-u_{h}\right\|$ & Order & $\max _{F \in \mathcal{F}_{h}^{\mathrm{i}}}\left\|\mathbf{A}_{\mathfrak{g}_{F}}^{-1}\right\|_{\infty}$ & $\operatorname{cg}+$ AMG it. \\
\hline $8 \times 8$ & $1.6679 \mathrm{e}-2$ & - & $3.2200 \mathrm{e}-2$ & - & $1.1656 \mathrm{e}+1$ & 6 \\
$16 \times 16$ & $5.0746 \mathrm{e}-3$ & 1.72 & $1.8484 \mathrm{e}-2$ & 0.80 & $1.2094 \mathrm{e}+1$ & 7 \\
$32 \times 32$ & $1.5348 \mathrm{e}-3$ & 1.72 & $1.0777 \mathrm{e}-2$ & 0.78 & $1.2301 \mathrm{e}+1$ & 8 \\
$64 \times 64$ & $4.6235 \mathrm{e}-4$ & 1.73 & $6.2691 \mathrm{e}-3$ & 0.78 & $1.2401 \mathrm{e}+1$ & 9 \\
$128 \times 128$ & $1.4042 \mathrm{e}-4$ & 1.72 & $3.6333 \mathrm{e}-3$ & 0.79 & $1.2451 \mathrm{e}+1$ & 10 \\
\hline
\end{tabular}

$\boldsymbol{\kappa}_{\mid \Omega \backslash S_{1}}=k_{2} \mathbf{1}_{d}$. Using polar coordinates $(r, \theta)$ with $\theta=\cos ^{-1}(x / r)$ and origin at the center of Figure $3 \mathrm{~b}$, the first solution is given by

$$
u= \begin{cases}r^{\alpha} \cos (\alpha(\theta-\pi / 3)) & \text { if } \theta \in[0,2 \pi / 3), \\ r^{\alpha} \beta \cos (4 \pi / 3-\theta) & \text { if } \theta \in[2 \pi / 3,2 \pi),\end{cases}
$$

where $\alpha=3 / \pi \tan ^{-1}(\sqrt{1+2 / \epsilon}), \beta=\cos \left(\alpha^{\pi} / 3\right) / \cos \left(2 \alpha^{\pi} / 3\right)$ and $\epsilon=k_{1} / k_{2}$ is the heterogeneity ratio taken equal to 0.1. It can be proven that $u \in H^{2.29}(\Omega)$. The convergence results for this case are collected in Table 2. A second solution with less regularity is the following:

$$
u= \begin{cases}r^{\alpha} \sin (\alpha(\theta-\pi / 3)) & \text { if } \theta \in[0,2 \pi / 3) \\ r^{\alpha} \beta \sin (\alpha(4 \pi / 3-\theta)) & \text { if } \theta \in[2 \pi / 3,2 \pi)\end{cases}
$$

where now $\alpha=3 / \pi \tan ^{-1}(\sqrt{1+2 \epsilon}), \beta=\left(2 \cos \left(\alpha^{\pi} / 3\right)\right)^{-1}$ and, as before, $\epsilon=k_{1} / k_{2}=0.1$. In this case, $u \in$ $H^{1.79}(\Omega)$. The convergence results collected in Table 3 show, in particular, that the order of convergence remains optimal even for solution that do not sit in $H^{2}(\Omega)$. To conclude, we consider a solution with an even stronger singularity at the origin. In this case $\boldsymbol{\kappa}_{\mid S_{1}}$ and $\boldsymbol{\kappa}_{\mid S_{3}}$ are constant and equal to $k_{1} \mathbf{1}_{d}$ whereas $\boldsymbol{\kappa}_{\mid S_{2}}$ and $\boldsymbol{\kappa}_{\mid S_{4}}$ are constant and equal to $k_{2} \mathbf{1}_{d}$. This solution satisfies $u(r, \theta)=-u(r, \theta-\pi)$ for $\theta \in[0, \pi)$ and reads

$$
u= \begin{cases}r^{\alpha} \cos (\alpha(\theta-\pi / 3)) & \text { if } \theta \in[0,2 \pi / 3), \\ r^{\alpha} \beta \sin (\alpha(5 \pi / 6-\theta)) & \text { if } \theta \in[2 \pi / 3, \pi),\end{cases}
$$

where $\alpha=6 / \pi \tan ^{-1}(1 / \sqrt{1+2 / \epsilon}), \beta=1 /\left(2 \cos \left(\alpha^{\pi} / 3\right)\right)$ and this time $\epsilon=k_{2} / k_{1}=1 / 30$. This solution belongs to $H^{1.29}(\Omega)$. The convergence results are collected in Table 4. Similar considerations as for the test case (5.3) hold.

\subsection{Steady incompressible Navier-Stokes equations}

The Kovasznay problem. To verify the asymptotic convergence properties of the method (4.7), we consider Kovasznay's analytical solution of the INS equations [39] on the square domain $\Omega=(-0.5,1.5) \times(0,2)$,

$$
u_{1}=1-\mathrm{e}^{\pi x_{2}} \cos \left(2 \pi x_{2}\right), \quad u_{2}=-1 / 2 \mathrm{e}^{\pi x_{1}} \sin \left(2 \pi x_{2}\right), \quad p=-1 / 2 \mathrm{e}^{\pi x_{1}} \cos \left(2 \pi x_{2}\right)-\bar{p},
$$


TABLE 4. Heterogeneous solution (5.4), $u \in H^{1.29}(\Omega)$.

\begin{tabular}{ccccccc}
\hline $\operatorname{card}\left(\mathcal{T}_{h}\right)$ & $\left\|u-u_{h}\right\|_{L^{2}(\Omega)}$ & Order & $\left\|u-u_{h}\right\|$ & Order & $\max _{F \in \mathcal{F}_{h}^{\mathrm{i}}}\left\|\mathbf{A}_{\mathfrak{g}_{F}}^{-1}\right\|_{\infty}$ & $\mathrm{cg}+$ AMG it. \\
\hline $8 \times 8$ & $3.5383 \mathrm{e}-2$ & - & $2.0444 \mathrm{e}-1$ & - & 1.2318 & 11 \\
$16 \times 16$ & $1.7796 \mathrm{e}-2$ & 0.99 & $1.6836 \mathrm{e}-1$ & 0.28 & 1.2416 & 16 \\
$32 \times 32$ & $8.0164 \mathrm{e}-3$ & 1.15 & $1.3747 \mathrm{e}-1$ & 0.29 & 1.2459 & 17 \\
$64 \times 64$ & $3.5358 \mathrm{e}-3$ & 1.18 & $1.1341 \mathrm{e}-1$ & 0.28 & 1.2480 & 20 \\
$128 \times 128$ & $1.5628 \mathrm{e}-3$ & 1.18 & $9.4240 \mathrm{e}-2$ & 0.27 & 1.2490 & 23 \\
\hline
\end{tabular}

TABLE 5. Convergence results for the Kovasznay problem.

\begin{tabular}{ccccccc}
\hline $\operatorname{card}\left(\mathcal{T}_{h}\right)$ & $\left\|u-u_{h}\right\|_{\left.L^{2}(\Omega)\right]^{d}}$ & Order & $\left\|p-p_{h}\right\|_{L^{2}(\Omega)}$ & Order & $\left\|u-u_{h}\right\|_{\mathrm{ns}}$ & Order \\
\hline 224 & $1.6539 \mathrm{e}-01$ & - & $2.5536 \mathrm{e}-01$ & - & $4.7777 \mathrm{e}-01$ & - \\
896 & $4.3732 \mathrm{e}-02$ & 1.92 & $1.0737 \mathrm{e}-01$ & 1.25 & $2.1759 \mathrm{e}-01$ & 1.13 \\
3584 & $1.1847 \mathrm{e}-02$ & 1.88 & $3.9802 \mathrm{e}-02$ & 1.43 & $1.0763 \mathrm{e}-01$ & 1.02 \\
14336 & $3.1620 \mathrm{e}-03$ & 1.91 & $1.7385 \mathrm{e}-02$ & 1.19 & $5.5182 \mathrm{e}-02$ & 0.96 \\
\hline
\end{tabular}

where $\bar{p}=\left\langle-\frac{1}{2} \mathrm{e}^{\pi x_{1}} \cos \left(2 \pi x_{2}\right)\right\rangle_{\Omega} \approx-0.920735694$ ensures the zero mean constraint for the pressure, $\nu=3 \pi$, and $f=0$ (the solution is nontrivial albeit $f=0$ since the boundary condition is non homogeneous). The example is run on a family of uniformly refined triangular meshes with mesh sizes ranging from 0.5 down to 0.03125 . According to Table 5, the errors $\left\|u-u_{h}\right\|_{\text {ns }}$ and $\left\|p-p_{h}\right\|_{L^{2}(\Omega)}$ converge to first order, while second order is attained for $\left\|u-u_{h}\right\|_{\left[L^{2}(\Omega)\right]^{d}}$.

The lid-driven cavity problem. To assess the behavior of the method in more complex situations we consider the classical lid-driven cavity problem. Despite its simple geometry, at large Reynolds numbers this problem presents complex flow patterns with counter-rotating vortices of significantly different scale. The domain is here the unit square with imposed horizontal velocity on the upper side and zero velocity on the others. In Figure 5 we provide the values of the velocity components on the centerlines of the domain. For the sake of completeness, we compare against the method of [22] with piecewise linear approximations of the velocity and the pressure. In both cases a uniform $128 \times 128$ Cartesian orthogonal mesh is used. The reference data of Erturk et al. [31] are also included for comparison. The proposed method shows essentially the same accuracy as the dG method of [22] at $R e=1000$. To observe more sizable differences, we also present the results for $R e=5000$ on the same mesh. In this case, where a slight loss of accuracy can be observed towards the boundaries of the domain.

Acknowledgements. The author is grateful to an anonymous referee for careful reading and constructive remarks. Discussions with Alexandre Ern (École des Ponts ParisTech) are also gratefully acknowledged.

\section{A. TeChnical Results}

\section{A.1. Proof of Theorem 3.8}

We start by proving the following technical result.

Lemma A.1 (consistency of the trace reconstruction). Let $v \in \mathcal{Q}_{P_{\Omega}, \kappa}$ and set $\mathbf{v}_{h}:=\mathcal{I}_{h}^{\mathrm{ccg}}(v)$ and $\left(v_{F}\right)_{F \in \mathcal{F}_{h}}=$ $\mathfrak{T}_{h}\left(\mathbf{v}_{h}\right)$. Under Assumption 3.7 there holds for all $h \in \mathcal{H}$, all $v \in \mathcal{Q}_{P_{\Omega}, \kappa}$, and all $F \in \mathcal{F}_{h}$,

$$
\left|v_{F}-v\left(\overline{\mathbf{x}}_{F}\right)\right| \leq C h_{T_{\mathfrak{g}_{F}}}^{2}
$$

where $C$ depends on $\boldsymbol{\kappa}$, on the mesh regularity parameters, on $\Lambda$, and on $\|v\|_{C^{2}\left(\mathcal{T}_{h}\right)}$.

Proof. The assertion is trivially verified for $F \in \mathcal{F}_{h}^{\mathrm{b}}$. Let now $F \in \mathcal{F}_{h}^{\mathrm{i}}$. Using the Taylor expansion of $v$ about $\mathbf{x}_{T_{\mathfrak{g}_{F}}}$ together with the fact that $T_{\mathfrak{g}_{F}}$ is star-shaped with respect to $\mathbf{x}_{T_{\mathfrak{g}_{F}}}$, we conclude that there exists 


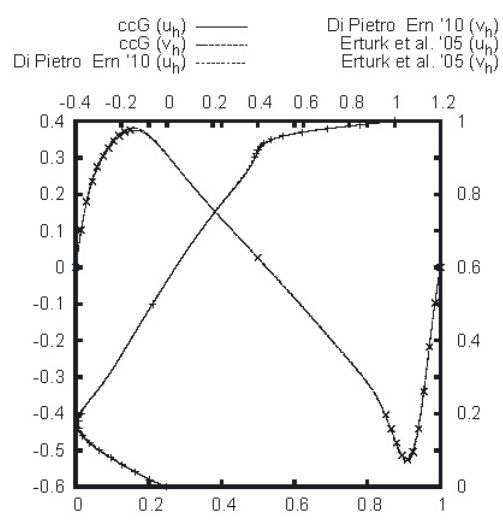

(a) $\operatorname{Re}=1000$

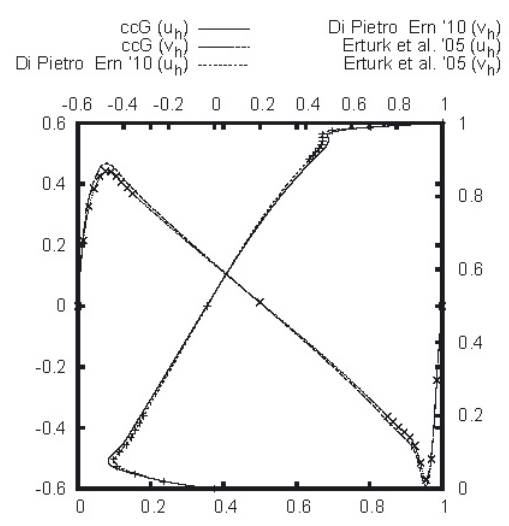

(b) $\operatorname{Re}=5000$

FiguRE 5. Lid-driven cavity problem, comparison of centerline velocity values (ccG $=$ present work, Di Pietro Ern '10 = Ref. [22], Erturk et al. '05 = Ref. [31]).

$\mathbf{y}\left(\overline{\mathbf{x}}_{F}\right) \in\left[\mathbf{x}_{T_{\mathfrak{g}_{F}}}, \mathbf{x}\right]$ such that

$$
v\left(\overline{\mathbf{x}}_{F}\right)=v\left(\mathbf{x}_{T_{\mathfrak{g}_{F}}}\right)+\nabla v\left(\mathbf{x}_{T_{\mathfrak{g}_{F}}}\right) \cdot\left(\overline{\mathbf{x}}_{F}-\mathbf{x}_{T_{\mathfrak{g}_{F}}}\right)+\frac{1}{2}\left(\overline{\mathbf{x}}_{F}-\mathbf{x}_{T_{\mathfrak{g}_{F}}}\right)^{t} \mathbf{H} v\left(\mathbf{y}\left(\overline{\mathbf{x}}_{F}\right)\right)\left(\overline{\mathbf{x}}_{F}-\mathbf{x}_{T_{\mathfrak{g}_{F}}}\right),
$$

where $\mathbf{H} v\left(\mathbf{y}\left(\overline{\mathbf{x}}_{F}\right)\right)$ denotes the Hessian of $v$ evaluated at $\mathbf{y}\left(\overline{\mathbf{x}}_{F}\right)$. Similarly, letting $\mathbf{v}_{h}=\mathcal{I}_{h}^{\mathrm{ccg}}(v)$ and $\left(v_{F}\right)_{F \in \mathcal{F}_{h}}:=$ $\mathfrak{T}_{h}\left(\mathbf{v}_{h}\right) \in \mathbb{R}^{\mathcal{F}_{h}}$,

$$
v_{F}=v\left(\mathbf{x}_{T_{\mathfrak{g}_{F}}}\right)+\nabla \xi_{\mathbf{v}_{h} \mid T_{\mathfrak{g}_{F}}}^{\mathfrak{g}_{F}} \cdot\left(\overline{\mathbf{x}}_{F}-\mathbf{x}_{T_{\mathfrak{g}_{F}}}\right) .
$$

Hence, $\left|v_{F}-v\left(\overline{\mathbf{x}}_{F}\right)\right| \leq h_{T}\left|\nabla \xi_{\mathbf{v}_{h}}^{\mathfrak{g}_{F}}\right| T_{\mathfrak{g}_{F}}-\nabla v\left(\overline{\mathbf{x}}_{F}\right) \mid+\frac{h_{T}^{2}}{2}\|v\|_{C^{2}(\bar{T})}$. The conclusion follows from Lemma 3.6.

We are now ready to prove Theorem 3.8. The proof closely follows that of [5], Lemma 3.10. Since it is clear that $\|w\|\left\|_{\boldsymbol{\kappa}, *} \leq \bar{\lambda}^{1 / 2}\right\| w \|_{*}$ for all $w \in H^{1}\left(\mathcal{T}_{h}\right)$, we only show the second inequality in (3.9). In the rest of the proof we abbreviate $a \lesssim b$ the inequality $a \leq C b$ where $C$ can depend on $\boldsymbol{\kappa}$, on the mesh regularity parameters, on $\Lambda$, and on $\|v\|_{C^{2}\left(\mathcal{T}_{h}\right)}$. Let $v \in \mathcal{Q}_{P_{\Omega}, \kappa}$ and set

$$
\mathbf{v}_{h}:=\mathcal{I}_{h}^{\operatorname{ccg}}(v) \in \mathbb{V}_{h}, \quad v_{h}:=\mathfrak{R}_{h}^{\mathrm{ccg}}\left(\mathbf{v}_{h}\right) \in V_{h}^{\mathrm{ccg}}, \quad\left(v_{F}\right)_{F \in \mathcal{F}_{h}}=\mathfrak{T}_{h}\left(\mathbf{v}_{h}\right) \in \mathbb{R}^{\mathcal{F}_{h}} .
$$

(i) Estimate of $\left\|\nabla v-\nabla_{h} v_{h}\right\|_{\left[L^{2}(\Omega)\right]^{d}}$. For all $T \in \mathcal{T}_{h}$ and all $\mathbf{x} \in T$ there holds

$$
\begin{aligned}
\nabla_{h} v_{h \mid T}-\nabla v(\mathbf{x})=\left\{\nabla v\left(\mathbf{x}_{T}\right)-\nabla v(\mathbf{x})\right\} & +\left\{\frac{1}{|T|_{d}} \sum_{F \in \mathcal{F}_{T}}|F|_{d-1}\left(v_{F}-v\left(\overline{\mathbf{x}}_{F}\right)\right) \mathbf{n}_{T, F}\right\} \\
& +\left\{\frac{1}{|T|_{d}} \sum_{F \in \mathcal{F}_{T}}|F|_{d-1}\left(v\left(\overline{\mathbf{x}}_{F}\right)-v\left(\mathbf{x}_{T}\right)\right) \mathbf{n}_{T, F}-\nabla v\left(\mathbf{x}_{T}\right)\right\}:=\mathfrak{T}_{1}+\mathfrak{T}_{2}+\mathfrak{T}_{3} .
\end{aligned}
$$

It is clear that $\left|\mathfrak{T}_{1}\right| \leq\|v\|_{C^{2}(\bar{T})} h_{T}$, hence $\sum_{T \in \mathcal{T}_{h}}\left\|\mathfrak{T}_{1}\right\|_{\left[L^{2}(T)\right]^{d}}^{2} \lesssim|\Omega|_{d} h^{2}$. Using Lemma A.1 together with the geometric relation (2.8) and mesh regularity it is inferred that

$$
\left|\mathfrak{T}_{2}\right| \leq \sum_{F \in \mathcal{F}_{T}} \frac{|F|_{d-1} d_{T, F}}{|T|_{d}} \frac{\left|v_{F}-v\left(\overline{\mathbf{x}}_{F}\right)\right|}{d_{T, F}} \lesssim d \frac{h_{T_{\mathfrak{g}_{F}}}^{2}}{d_{T, F}} \lesssim h_{T_{\mathfrak{g}_{F}}} .
$$


As a result, $\sum_{T \in \mathcal{T}_{h}}\left\|\mathfrak{T}_{2}\right\|_{\left[L^{2}(T)\right]^{d}}^{2} \lesssim|\Omega|_{d} h^{2}$. To handle the last term, we use the magic formula [5], equation (33): for all $T \in \mathcal{T}_{h}$ and all $\mathbf{x} \in T$,

$$
\frac{1}{|T|_{d}} \sum_{F \in \mathcal{F}_{T}}|F|_{d-1}\left(\overline{\mathbf{x}}_{F}-\mathbf{x}\right)_{i}\left(\mathbf{n}_{T, F}\right)_{j}=\delta_{i j}
$$

where $\delta_{i j}$ is Kronecker's symbol. By virtue of (A.1), there holds

$$
\mathfrak{T}_{3}=\frac{1}{|T|_{d}} \sum_{F \in \mathcal{F}_{T}}|F|_{d-1}\left[v\left(\overline{\mathbf{x}}_{F}\right)-v\left(\mathbf{x}_{T}\right)-\nabla v\left(\mathbf{x}_{T}\right) \cdot\left(\overline{\mathbf{x}}_{F}-\mathbf{x}_{T}\right)\right] \mathbf{n}_{T, F} \leq \sum_{F \in \mathcal{F}_{T}} \frac{|F|_{d-1} d_{T, F}}{|T|_{d}}\|v\|_{C^{2}(\bar{T})} h_{T} .
$$

Proceeding as for $\mathfrak{T}_{2}$, we easily infer that $\sum_{T \in \mathcal{T}_{h}}\left\|\mathfrak{T}_{3}\right\|_{\left[L^{2}(T)\right]^{d}}^{2} \lesssim|\Omega|_{d} h^{2}$, thereby concluding that

$$
\left\|\nabla v-\nabla_{h} v_{h}\right\|_{\left[L^{2}(\Omega)\right]^{d}} \lesssim h .
$$

(ii) Estimate of the remaining terms in $\|\cdot \mid\|_{*}$. By the continuous trace inequality (2.5),

$$
\left|v-v_{h}\right|_{\mathrm{J}}^{2} \lesssim \sum_{F \in \mathcal{F}_{h}} h_{F}^{-1} \sum_{T \in \mathcal{T}_{F}}\left\|\left(v-v_{h}\right)_{\mid T}\right\|_{L^{2}(F)}^{2} \leq C_{\mathrm{tr}, \mathrm{c}}^{2} \sum_{F \in \mathcal{F}_{h}} h_{F}^{-1} \sum_{T \in \mathcal{T}_{F}}\left\{h_{T}^{-1}\left\|v-v_{h}\right\|_{L^{2}(T)}^{2}+h_{T}\left|v-v_{h}\right|_{H^{1}(T)}^{2}\right\} .
$$

On the other hand, for all $T \in \mathcal{T}_{h}$, expanding $v$ at $\mathbf{x}_{T}$ and using the previous point to estimate $\left|\nabla v_{h}-\nabla v\left(\mathbf{x}_{T}\right)\right|$, it is proven that $\left\|v-v_{h}\right\|_{L^{2}(T)} \lesssim h_{T}^{2}$. It follows easily that $\left|v-v_{h}\right|_{J} \lesssim h$. The term $\sum_{T \in \mathcal{T}_{h}} h_{T}\left\|\nabla\left(v-v_{h}\right)_{\mid T} \cdot \mathbf{n}_{T}\right\|_{L^{2}(\partial T)}$ can be handled in a similar way using the trace inequality (2.5), thereby concluding the proof.

\section{A.2. Proof of Theorem 3.11}

Let $v \in V \cap H^{2}(\Omega)$, and set $\mathbf{v}_{h}:=\mathcal{I}_{h}^{\text {ccg }}(v) \in \mathbb{V}_{h}$ and $\left(v_{F}\right)_{F \in \mathcal{F}_{h}}=\mathfrak{T}_{h}\left(\mathbf{v}_{h}\right) \in \mathbb{R}^{\mathcal{F}_{h}}$. In the rest of the proof we abbreviate $a \lesssim b$ the inequality $a \leq C b$ where $C$ can depend on the mesh regularity parameters but not on the meshsize $h$.

(i) Estimate of $\left\|\nabla v-\nabla_{h} v_{h}\right\|_{\left[L^{2}(\Omega)\right]^{d}}$. The quantity to estimate is decomposed as follows:

$$
\begin{aligned}
\left\|\nabla v-\nabla_{h} v_{h}\right\|_{\left[L^{2}(\Omega)\right]^{d}}^{2} & =\sum_{T \in \mathcal{T}_{h}} \sum_{F \in \mathcal{F}_{T}} \int_{\mathcal{P}_{T, F}}\left|\nabla v-\nabla \xi_{\mathbf{v}_{h}}^{\mathfrak{g}_{F}}+\nabla \xi_{\mathbf{v}_{h}}^{\mathfrak{g}_{F}}-\nabla v_{h}\right|^{2} \\
& \lesssim \sum_{T \in \mathcal{T}_{h}} \sum_{F \in \mathcal{F}_{T}} \int_{\mathcal{P}_{T, F}}\left|\nabla v-\nabla \xi_{\mathbf{v}_{h}}^{\mathfrak{g}_{F}}\right|^{2}+\sum_{T \in \mathcal{T}_{h}} \sum_{F \in \mathcal{F}_{T}} \int_{\mathcal{P}_{T, F}}\left|\nabla \xi_{\mathbf{v}_{h}}^{\mathfrak{g}_{F}}-\nabla v_{h}\right|^{2}:=\mathfrak{T}_{1}+\mathfrak{T}_{2} .
\end{aligned}
$$

For the first term it suffices to use Assumption 3.10 to infer

$$
\mathfrak{T}_{1}=\sum_{T \in \mathcal{T}_{h}} \sum_{F \in \mathcal{F}_{T}}\left|v-\xi_{\mathbf{v}_{h}}^{\mathfrak{g}_{F}}\right|_{H^{1}\left(\mathcal{P}_{T, F}\right)}^{2} \lesssim h^{2}|v|_{H^{2}(\Omega)}^{2} .
$$

To estimate the second term, preliminarily observe that, for all $T \in \mathcal{T}_{h}$,

$$
\nabla_{h} v_{h \mid T}=\sum_{F^{\prime} \in \mathcal{F}_{T}} \frac{\left|F^{\prime}\right|_{d-1}}{|T|_{d}}\left(\xi_{\mathbf{v}_{h}^{\prime}}^{\mathfrak{g}_{F^{\prime}} \mid \mathcal{P}_{T, F^{\prime}}}\left(\overline{\mathbf{x}}_{F^{\prime}}\right)-v_{T}\right) \mathbf{n}_{T, F^{\prime}}=\sum_{F^{\prime} \in \mathcal{F}_{T}} \frac{\left|F^{\prime}\right|_{d-1}}{|T|_{d}} \nabla \xi_{\mathbf{v}_{h}}^{\mathfrak{g}_{F^{\prime}}} \mathcal{P}_{T, F^{\prime}} \cdot\left(\overline{\mathbf{x}}_{F^{\prime}}-\mathbf{x}_{T}\right) \mathbf{n}_{T, F^{\prime}}
$$

where we have used the linearity of $\xi_{\mathbf{v}_{h}}^{\mathfrak{g}_{F^{\prime}}} \mid \mathcal{P}_{T, F^{\prime}}$ together with the fact that $\xi_{\mathbf{v}_{h}}^{\mathfrak{g}_{F^{\prime}}} \mid \mathcal{P}_{T, F^{\prime}}\left(\mathbf{x}_{T}\right)=v_{T}$ to infer that $\xi_{\mathbf{v}_{h}}^{\mathfrak{g}_{F^{\prime}}}\left|\mathcal{P}_{T, F^{\prime}}\left(\overline{\mathbf{x}}_{F}\right)=v_{T}+\nabla \xi_{\mathbf{v}_{h}}^{\mathfrak{g}_{F^{\prime}}}\right| \mathcal{P}_{T, F^{\prime}} \cdot\left(\overline{\mathbf{x}}_{F^{\prime}}-\mathbf{x}_{T}\right)$. Using formula (A.1) together with the definition of the gradient 
reconstruction (2.13), we obtain

$$
\langle\nabla v\rangle_{T}-\nabla_{h} v_{h \mid T}=\sum_{F^{\prime} \in \mathcal{F}_{T}} \frac{\left|F^{\prime}\right|_{d-1}}{|T|_{d}}\left(\langle\nabla v\rangle_{T}-\nabla \xi_{\mathbf{v}_{h}^{\prime}}^{\mathfrak{g}_{F^{\prime}} \mathcal{P}_{T, F^{\prime}}}\right) \cdot\left(\overline{\mathbf{x}}_{F^{\prime}}-\mathbf{x}_{T}\right) \mathbf{n}_{T, F^{\prime}}
$$

Exploiting (A.3), we estimate $\left|\mathfrak{T}_{2}\right|$ as follows:

$$
\begin{aligned}
\left|\mathfrak{T}_{2}\right| \lesssim & \sum_{T \in \mathcal{T}_{h}} \sum_{F \in \mathcal{F}_{T}}\left|\mathcal{P}_{T, F}\right|_{d}\left|\nabla \xi_{\mathbf{v}_{h}}^{\mathfrak{g}_{F}}\right| \mathcal{P}_{T, F}-\left.\langle\nabla v\rangle_{\mathcal{P}_{T, F}}\right|^{2}+\sum_{T \in \mathcal{T}_{h}} \sum_{F \in \mathcal{F}_{T}}\left|\mathcal{P}_{T, F}\right|_{d}\left|\langle\nabla v\rangle_{\mathcal{P}_{T, F}}-\langle\nabla v\rangle_{T}\right|^{2} \\
& +\sum_{T \in \mathcal{T}_{h}} \sum_{F \in \mathcal{F}_{T}}\left|\mathcal{P}_{T, F}\right|_{d}\left|\sum_{F^{\prime} \in \mathcal{F}_{T}} \frac{\left|F^{\prime}\right|_{d-1}}{|T|_{d}}\left(\langle\nabla v\rangle_{\mathcal{P}_{T, F^{\prime}}}-\nabla \xi_{\mathbf{v}_{h}}^{\mathfrak{g}_{F^{\prime}}} \mid \mathcal{P}_{T, F^{\prime}}\right) \cdot\left(\overline{\mathbf{x}}_{F^{\prime}}-\mathbf{x}_{T}\right) \mathbf{n}_{T, F^{\prime}}\right|^{2} \\
& +\sum_{T \in \mathcal{T}_{h}} \sum_{F \in \mathcal{F}_{T}}\left|\mathcal{P}_{T, F}\right|_{d}\left|\sum_{F^{\prime} \in \mathcal{F}_{T}} \frac{\left|F^{\prime}\right|_{d-1}}{|T|_{d}}\left(\langle\nabla v\rangle_{T}-\langle\nabla v\rangle_{\mathcal{P}_{T, F^{\prime}}}\right) \cdot\left(\overline{\mathbf{x}}_{F^{\prime}}-\mathbf{x}_{T}\right) \mathbf{n}_{T, F^{\prime}}\right|^{2}:=\mathfrak{T}_{2,1}+\mathfrak{T}_{2,2}+\mathfrak{T}_{2,3}+\mathfrak{T}_{2,4}
\end{aligned}
$$

The Cauchy-Schwarz inequality yields

$$
\nabla \xi_{\mathbf{v}_{h} \mid \mathcal{P}_{T, F}}^{\mathfrak{g}_{F}}-\langle\nabla v\rangle_{\mathcal{P}_{T, F}}=\frac{1}{\left|\mathcal{P}_{T, F}\right|_{d}} \int_{\mathcal{P}_{T, F}}\left(\nabla \xi_{\mathbf{v}_{h}}^{\mathfrak{g}_{F}}-\nabla v\right) \leq \frac{1}{\left|\mathcal{P}_{T, F}\right|_{d}^{1 / 2}}\left|\xi_{\mathbf{v}_{h}}^{\mathfrak{g}_{F}}-v\right|_{H^{1}\left(\mathcal{P}_{T, F}\right)}
$$

whence, by Assumption 3.10,

$$
\mathfrak{T}_{2,1} \leq \sum_{T \in \mathcal{T}_{h}} \sum_{F \in \mathcal{F}_{T}}\left|\xi_{\mathbf{v}_{h}}^{\mathfrak{g}_{F}}-v\right|_{H^{1}\left(\mathcal{P}_{T, F}\right)}^{2} \lesssim h^{2}|v|_{H^{2}(\Omega)}^{2}
$$

The term $\mathfrak{T}_{2,2}$ can be estimated in a similar fashion using the fact that

$$
\langle\nabla v\rangle_{\mathcal{P}_{T, F}}-\langle v\rangle_{T}=\frac{1}{\left|\mathcal{P}_{T, F}\right|_{d}} \int_{\mathcal{P}_{T, F}}\left(\nabla v-\langle\nabla v\rangle_{T}\right)
$$

and the approximation properties of the $L^{2}$-projection of $\nabla v$ onto $\left[\mathbb{P}_{d}^{0}\left(\mathcal{T}_{h}\right)\right]^{d}$ to conclude. To estimate $\mathfrak{T}_{2,3}$, observe preliminarily that, for all $T \in \mathcal{T}_{h}$ and all $F^{\prime} \in \mathcal{F}_{T}$, equation (2.7) yields

$$
\left|F^{\prime}\right|_{d-1}\left(\langle\nabla v\rangle_{\mathcal{P}_{T, F^{\prime}}}-\nabla \xi_{\mathbf{v}_{h}}^{\mathfrak{g}_{F^{\prime}} \mid \mathcal{P}_{T, F^{\prime}}}\right)=\frac{d}{d_{T, F^{\prime}}} \int_{\mathcal{P}_{T, F^{\prime}}}\left(\nabla v-\nabla \xi_{\mathbf{v}_{h}}^{\mathfrak{g}_{F^{\prime}}}\right)
$$

The Cauchy-Schwarz inequality together with (2.6) yield

$$
\begin{aligned}
\mid \sum_{F^{\prime} \in \mathcal{F}_{T}} \frac{\left|F^{\prime}\right|_{d-1}}{|T|_{d}} & \left.\left(\langle\nabla v\rangle_{\mathcal{P}_{T, F^{\prime}}}-\nabla \xi_{\mathbf{v}_{h}}^{\mathfrak{g}_{F^{\prime}}} \mathcal{P}_{T, F^{\prime}}\right) \cdot\left(\overline{\mathbf{x}}_{F^{\prime}}-\mathbf{x}_{T}\right)\right|^{2} \leq \frac{d^{2} h_{T}^{2}}{d_{T, F^{\prime}}^{2}|T|_{d}^{2}}\left|\sum_{F^{\prime} \in \mathcal{F}_{T}} \int_{\mathcal{P}_{T, F^{\prime}}}\right| \nabla v-\nabla \xi_{\mathbf{v}_{h}}^{\mathfrak{g}_{F^{\prime}}}||^{2} \\
\leq & \frac{d^{2} h_{T}^{2}}{d_{T, F^{\prime}}^{2}|T|_{d}^{2}}\left\{\sum_{F^{\prime} \in \mathcal{F}_{T}}\left|\mathcal{P}_{T, F^{\prime}}\right|_{d}\right\} \times\left\{\sum_{F^{\prime} \in \mathcal{F}_{T}}\left|v-\xi_{\mathbf{v}_{h}}^{\mathfrak{g}_{F^{\prime}}}\right|_{H^{1}\left(\mathcal{P}_{T, F^{\prime}}\right)}^{2}\right\}=\frac{d^{2}}{|T|_{d} \varrho_{3}^{2}} \sum_{F^{\prime} \in \mathcal{F}_{T}}\left|v-\xi_{\mathbf{v}_{h}}^{\mathfrak{g}_{F^{\prime}}}\right|_{H^{1}\left(\mathcal{P}_{T, F^{\prime}}\right)}^{2}
\end{aligned}
$$


We therefore have

$$
\begin{aligned}
\mathfrak{T}_{2,3} & \leq \frac{d^{2}}{\varrho_{3}^{2}} \sum_{T \in \mathcal{T}_{h}} \sum_{F \in \mathcal{F}_{T}}\left\{\frac{\left|\mathcal{P}_{T, F}\right|_{d}}{|T|_{d}} \sum_{F^{\prime} \in \mathcal{F}_{T}}\left|v-\xi_{\mathbf{v}_{h}}^{\mathfrak{g}_{F^{\prime}}}\right|_{H^{1}\left(\mathcal{P}_{T, F^{\prime}}\right)}^{2}\right\} \\
& =\frac{d^{2}}{\varrho_{3}^{2}} \sum_{T \in \mathcal{T}_{h}}\left\{\sum_{F \in \mathcal{F}_{T}} \frac{\left|\mathcal{P}_{T, F}\right|_{d}}{|T|_{d}}\right\} \times\left\{\sum_{F^{\prime} \in \mathcal{F}_{T}}\left|v-\xi_{\mathbf{v}_{h}}^{\mathfrak{g}_{F^{\prime}}}\right|_{H^{1}\left(\mathcal{P}_{T, F^{\prime}}\right)}^{2}\right\}=\frac{d^{2}}{\varrho_{3}^{2}} \sum_{T \in \mathcal{T}_{h}} \sum_{F^{\prime} \in \mathcal{F}_{T}}\left|v-\xi_{\mathbf{v}_{h}}^{\mathfrak{g}_{F^{\prime}}}\right|_{H^{1}\left(\mathcal{P}_{T, F^{\prime}}\right.}^{2},
\end{aligned}
$$

and, by Assumption 3.10, we infer that $\mathfrak{T}_{2,3} \lesssim h^{2}\|v\|_{H^{2}(\Omega)}^{2}$. Similar geometric manipulations allow to prove that $\mathfrak{T}_{2,4} \lesssim h^{2}\|v\|_{H^{2}(\Omega)}^{2}$. In conclusion,

$$
\left\|\nabla v-\nabla_{h} v_{h}\right\|_{\left[L^{2}(\Omega)\right]^{d}} \lesssim h\|v\|_{H^{2}(\Omega)}
$$

(ii) Estimate of the remaining terms in $\|\cdot \mid\|_{*}$. We start by estimating $\left\|v-v_{h}\right\|_{L^{2}(\Omega)}$ in view of applying the trace inequality (2.5). Let $w_{h} \in \mathbb{P}_{d}^{1}\left(\mathcal{S}_{h}\right)$ be such that

$$
\forall T \in \mathcal{T}_{h}, \forall F \in \mathcal{F}_{T}, \quad w_{h \mid \mathcal{P}_{T, F}}=\xi_{\mathbf{v}_{h} \mid \mathcal{P}_{T, F}}^{\mathfrak{g}_{F}}
$$

The triangle inequality yields

$$
\left\|v-v_{h}\right\|_{L^{2}(\Omega)} \leq\left\|v-w_{h}\right\|_{L^{2}(\Omega)}+\left\|w_{h}-v_{h}\right\|_{L^{2}(\Omega)}:=\mathfrak{T}_{1}+\mathfrak{T}_{2} .
$$

By Assumption 3.10, $\mathfrak{T}_{1} \lesssim h^{2}\|v\|_{H^{2}(\Omega)}$. To estimate the second term, observe that for all $T \in \mathcal{T}_{h}$, all $F \in \mathcal{F}_{T}$ and all $\mathbf{x} \in \mathcal{P}_{T, F}$,

$$
\xi_{\mathbf{v}_{h} \mid \mathcal{P}_{T, F}}^{\mathfrak{g}_{F}}(\mathbf{x})=v_{T}+\left(\nabla \xi_{\mathbf{v}_{h}}^{\mathfrak{g}_{F}}\right)_{\mathcal{P}_{T, F}} \cdot\left(\mathbf{x}-\mathbf{x}_{T}\right)
$$

since, by construction, $\xi_{\mathbf{v}_{h}}^{\mathfrak{g}_{F}} \mathcal{P}_{T, F}\left(\mathbf{x}_{T}\right)=v_{T}$. As a consequence, by virtue of (A.4), (2.14) and the triangular inequality, there holds

$$
\mathfrak{T}_{2}^{2}=\sum_{T \in \mathcal{T}_{h}} \sum_{F \in \mathcal{F}_{T}}\left\|w_{h}-v_{h}\right\|_{L^{2}\left(\mathcal{P}_{T, F}\right)}^{2} \leq h \sum_{T \in \mathcal{T}_{h}} \sum_{F \in \mathcal{F}_{T}}\left\|\nabla \xi_{\mathbf{v}_{h}}^{\mathfrak{g}_{F}}-\nabla v_{h}\right\|_{\left[L^{2}\left(\mathcal{P}_{T, F}\right)\right]^{d}}^{2}
$$

Proceeding as for the second term in the right-hand side of (A.2), we infer that $\mathfrak{T}_{2} \lesssim h^{2}|v|_{H^{2}(\Omega)}$. Therefore,

$$
\left\|v-v_{h}\right\|_{L^{2}(\Omega)} \lesssim h^{2}\|v\|_{H^{2}(\Omega)}
$$

To bound $\left|v-v_{h}\right|_{\mathrm{J}}$ and $\sum_{T \in \mathcal{T}_{h}} h_{T}\left\|\nabla(v)_{\mid T} \cdot \mathbf{n}_{F}\right\|_{L^{2}(\partial T)}$ use the continuous trace inequality (2.5) as in point (ii) of Theorem 3.8 together with (A.5).

\section{A.3. Proof of Lemma 4.2}

In the proof we abbreviate $a \lesssim b$ the inequality $a \leq C b$ where $C$ can depend on the mesh regularity parameters and on $\Omega$ but not on the meshsize $h$. By definition,

$$
\left\|v_{h}\right\|^{2}=\left\|\nabla_{h} v_{h}\right\|_{\left[L^{2}(\Omega)\right]^{d}}^{2}+\left|v_{h}\right|_{\mathrm{J}}^{2}:=\mathfrak{T}_{1}+\mathfrak{T}_{2}
$$

(i) Bound for $\mathfrak{T}_{1}$. The first term can be bounded as follows:

$$
\left.\left.\mathfrak{T}_{1} \lesssim \sum_{T \in \mathcal{T}_{h}}|T|_{d}\left|\frac{1}{|T|_{d}} \sum_{F \in \mathcal{F}_{T}}\right| F\right|_{d-1}\left(\langle v\rangle_{T}-\langle v\rangle_{F}\right) \mathbf{n}_{T, F}\right|^{2}+\left.\left.\sum_{T \in \mathcal{T}_{h}}|T|_{d}\left|\frac{1}{|T|_{d}} \sum_{F \in \mathcal{F}_{T}}\right| F\right|_{d-1}\left(\langle v\rangle_{F}-\left\langle\xi_{\mathbf{v}_{h}}^{\mathfrak{g}_{F}}\right\rangle_{F}\right) \mathbf{n}_{T, F}\right|^{2} .
$$


Denote by $\mathfrak{T}_{1,1}$ and $\mathfrak{T}_{1,2}$ the addends in the right-hand side. Using the Cauchy-Schwarz inequality together with formula $(2.8)$

$\mathfrak{T}_{1,1} \leq \sum_{T \in \mathcal{T}_{h}}\left\{\left(\sum_{F \in \mathcal{F}_{T}} \frac{|F|_{d-1}}{|T|_{d}} d_{T, F}\right) \times\left(\sum_{F \in \mathcal{F}_{T}} d_{T, F}^{-1}\left\|\langle v\rangle_{T}-v\right\|_{L^{2}(F)}^{2}\right)\right\} \lesssim d \sum_{T \in \mathcal{T}_{h}} \sum_{F \in \mathcal{F}_{T}} \frac{h_{T}}{d_{T, F}}\|v\|_{H^{1}(T)}^{2} \lesssim\|v\|_{H^{1}(\Omega)}^{2}$,

where we have used the classical estimate

$$
\left\|\langle v\rangle_{T}-v\right\|_{L^{2}(F)}=\left\|\pi_{h}^{0} v-v\right\|_{L^{2}(F)} \lesssim h_{T}^{1 / 2}|v|_{H^{1}(T)} .
$$

As for the term $\mathfrak{T}_{1,2}$, repeated applications of the Cauchy-Schwarz inequality together with (2.8) yield

$$
\mathfrak{T}_{1,2} \leq d \sum_{T \in \mathcal{T}_{h}} \sum_{F \in \mathcal{F}_{T}} \frac{1}{d_{T, F}}\left\|v-\xi_{\mathbf{v}_{h}}^{\mathfrak{g}_{F}}\right\|_{L^{2}(F)}^{2} .
$$

Since $\boldsymbol{\kappa}=\nu \mathbf{1}_{d}$, for all $F \in \mathcal{F}_{h}^{\mathbf{i}}$ and all $\mathbf{x} \in F, \xi_{\mathbf{v}_{h}}^{\mathfrak{g}_{F}}(\mathbf{x})$ can be expressed as a linear combination of the values $\left\{\langle v\rangle_{T}\right\}_{T \in \mathcal{T}_{\mathfrak{g}}}$ with coefficients $\left\{\tau_{T}^{F}(\mathbf{x})\right\}_{T \in \mathcal{T}_{\mathfrak{g}}}$ equal to the barycentric coordinates of $\mathbf{x}$ with respect to the simplex $S_{\mathfrak{g}}$ (cf. Fig. 1). Clearly, there exists $C_{\Lambda}<+\infty$ depending on $\Lambda$ in Assumption 3.7 and on the mesh regularity parameters but independent of $h$ such that $\max _{\mathbf{x} \in F} \max _{T \in \mathcal{T}_{\mathfrak{g}}}\left|\tau_{T}^{F}(\mathbf{x})\right| \leq C_{\Lambda}$. Hence, for all $F \in \mathcal{F}_{h}^{\mathrm{i}}$, using the triangular inequality, the continuous trace inequality (2.5), and (A.6) we infer

$$
\left\|v-\xi_{\mathbf{v}_{h}}^{\mathfrak{g}_{F}}\right\|_{L^{2}(F)} \leq \sum_{T \in \mathcal{T}_{\mathfrak{g}}}\left(\max _{\mathbf{x} \in F}\left|\tau_{T}^{F}(\mathbf{x})\right|\right)\left\|v-\langle v\rangle_{T}\right\|_{L^{2}(F)} \lesssim C_{\Lambda} \sum_{T \in \mathcal{T}_{\mathfrak{g}}} \tau_{T}^{F} h_{T}^{1 / 2}|v|_{H^{1}(T)} .
$$

On boundary faces $F \in \mathcal{F}_{h}^{\mathrm{b}}$ both $\xi_{\mathbf{v}_{h}}^{\mathfrak{g}_{F}}$ (by definition, $c f$. point (i) of Section 2.3) and $v$ vanish, hence $\| v-$ $\xi_{\mathbf{v}_{h}}^{\mathfrak{g}_{F}} \|_{L^{2}(F)}=0$. We conclude that $\mathfrak{T}_{1,2} \lesssim\|v\|_{H^{1}(\Omega)}$.

(ii) Bound for $\mathfrak{T}_{2}$. Using the fact that $\llbracket v \rrbracket_{F}(\mathbf{x})=0$ for all $F \in \mathcal{F}_{h}$ and all $\mathbf{x} \in F$ together with the continuous trace inequality (2.5) and the bound (2.3) there holds

$$
\begin{aligned}
\left|v_{h}\right|_{J}^{2} & =\sum_{F \in \mathcal{F}_{h}}\left\|\llbracket v_{h}-v \rrbracket\right\|_{L^{2}(F)}^{2} \lesssim \sum_{F \in \mathcal{F}_{h}} \sum_{T \in \mathcal{F}_{T}} h_{F}^{-1}\left\|\left(v-v_{h}\right)_{\mid T}\right\|_{L^{2}(F)}^{2} \\
& \lesssim \sum_{F \in \mathcal{F}_{h}} \sum_{T \in \mathcal{T}_{F}} h_{T}^{-2}\left\|v-v_{h}\right\|_{L^{2}(T)}^{2}+\sum_{F \in \mathcal{F}_{h}} \sum_{T \in \mathcal{T}_{F}}\left\|\nabla\left(v-v_{h}\right)\right\|_{\left[L^{2}(T)\right]^{d}}^{2}:=\mathfrak{T}_{2,1}+\mathfrak{T}_{2,2} .
\end{aligned}
$$

To estimate the first term, use (2.14) together with the approximation properties of $\mathcal{T}_{h}$ to infer, for all $T \in \mathcal{T}_{h}$,

$$
\left\|v-v_{h}\right\|_{L^{2}(T)}^{2}=\int_{T}\left(v_{T}+\mathfrak{G}_{h}\left(\mathbf{v}_{h}\right)_{\mid T} \cdot\left(\mathbf{x}-\mathbf{x}_{T}\right)-v(\mathbf{x})\right)^{2} \mathrm{~d} \mathbf{x} \lesssim\left\|\langle v\rangle_{T}-v\right\|_{L^{2}(T)}^{2}+h_{T}\left\|\nabla v_{h}\right\|_{\left[L^{2}(T)\right]^{d}}^{2} .
$$

Using point (i) it is inferred that $\mathfrak{T}_{2,1} \lesssim\|\nabla u\|_{\left[L^{2}(\Omega)\right]^{d}}^{2}$. To conclude the proof use again point (i) to estimate $\mathfrak{T}_{2,2} \lesssim\|\nabla u\|_{\left[L^{2}(\Omega)\right]^{d}}^{2}$

\section{REFERENCES}

[1] I. Aavatsmark, T. Barkve, Ø. Bøe and T. Mannseth, Discretization on unstructured grids for inhomogeneous, anisotropic media, Part I: Derivation of the methods. SIAM J. Sci. Comput. 19 (1998) 1700-1716.

[2] I. Aavatsmark, T. Barkve, $\varnothing$. Bøe and T. Mannseth, Discretization on unstructured grids for inhomogeneous, anisotropic media, Part II: Discussion and numerical results. SIAM J. Sci. Comput. 19 (1998) 1717-1736.

[3] I. Aavatsmark, G.T. Eigestad, B.T. Mallison and J.M. Nordbotten, A compact multipoint flux approximation method with improved robustness. Numer. Methods Partial Differential Equations 24 (2008) 1329-1360. 
[4] L. Agélas, D.A. Di Pietro and J. Droniou, The G method for heterogeneous anisotropic diffusion on general meshes. ESAIM: M2AN 44 (2010) 597-625.

[5] L. Agélas, D.A. Di Pietro, R. Eymard and R. Masson, An abstract analysis framework for nonconforming approximations of diffusion problems on general meshes. IJFV 7 (2010) 1-29.

[6] D.N. Arnold, An interior penalty finite element method with discontinuous elements. SIAM J. Numer. Anal. 19 (1982) $742-760$.

[7] D.N. Arnold, F. Brezzi, B. Cockburn and L.D. Marini, Unified analysis of discontinuous Galerkin methods for elliptic problems. SIAM J. Numer. Anal. 39 (2002) 1749-1779.

[8] J.-P. Aubin, Analyse fonctionnelle appliquée. Presses Universitaires de France, Paris (1987).

[9] L. Botti and D.A. Di Pietro, A pressure-correction scheme for convection-dominated incompressible flows with discontinuous velocity and continuous pressure. J. Comput. Phys. 230 (2011) 572-585.

[10] S.C. Brenner and L.R. Scott, The mathematical theory of finite element methods, Texts in Applied Mathematics, 3th edition 15. Springer, New York (2008).

[11] F. Brezzi, K. Lipnikov and M. Shashkov, Convergence of mimetic finite difference methods for diffusion problems on polyhedral meshes. SIAM J. Numer. Anal. 45 (2005) 1872-1896.

[12] F. Brezzi, K. Lipnikov and V. Simoncini, A family of mimetic finite difference methods on polygonal and polyhedral meshes. Math. Models Methods Appl. Sci. 15 (2005) 1533-1553.

[13] F. Brezzi, G. Manzini, L.D. Marini, P. Pietra and A. Russo, Discontinuous Galerkin approximations for elliptic problems. Numer. Methods Partial Differential Equations 16 (2000) 365-378.

[14] A. Buffa and C. Ortner, Compact embeddings of broken Sobolev spaces and applications. IMA J. Numer. Anal. 4 (2009) $827-855$.

[15] E. Burman and A. Ern, Continuous interior penalty $h p$-finite element methods for advection and advection-diffusion equations. Math. Comp. 76 (2007) 1119-1140.

[16] E. Burman and P. Zunino, A domain decomposition method for partial differential equations with non-negative form based on interior penalties. SIAM J. Numer. Anal. 44 (2006) 1612-1638.

[17] Y. Cao, R. Helmig and B.I. Wohlmuth, Geometrical interpretation of the multi-point flux approximation L-method. Internat. J. Numer. Methods Fluids 60 (2009) 1173-1199.

[18] P.G. Ciarlet, The finite element method for elliptic problems, Classics in Applied Mathematics 40. Society for Industrial and Applied Mathematics (SIAM), Philadelphia, PA (2002). Reprint of the 1978 original [North-Holland, Amsterdam; MR0520174 (58 \#25001)].

[19] D.A. Di Pietro, Analysis of a discontinuous Galerkin approximation of the Stokes problem based on an artificial compressibility flux. Internat. J. Numer. Methods Fluids 55 (2007) 793-813.

[20] D.A. Di Pietro, Cell centered Galerkin methods. C. R. Acad. Sci. Paris, Sér. I 348 (2010) $31-34$.

[21] D.A. Di Pietro, A compact cell-centered Galerkin method with subgrid stabilization. C. R. Acad. Sci. Paris, Sér. I 349 (2011) 93-98.

[22] D.A. Di Pietro and A. Ern, Discrete functional analysis tools for discontinuous Galerkin methods with application to the incompressible Navier-Stokes equations. Math. Comp. 79 (2010) 1303-1330.

[23] D.A. Di Pietro and A. Ern, Analysis of a discontinuous Galerkin method for heterogeneous diffusion problems with lowregularity solutions. Numer. Methods Partial Differential Equations (2011). Published online, DOI: 10.1002/num.20675.

[24] D.A. Di Pietro and A. Ern, Mathematical aspects of discontinuous Galerkin methods, Mathematics and Applications 69. Springer-Verlag, Berlin (2011). In press.

[25] D.A. Di Pietro, A. Ern and J.-L. Guermond, Discontinuous Galerkin methods for anisotropic semi-definite diffusion with advection. SIAM J. Numer. Anal. 46 (2008) 805-831.

[26] J. Droniou and R. Eymard, A mixed finite volume scheme for anisotropic diffusion problems on any grid. Numer. Math. 105 (2006) 35-71.

[27] J. Droniou, R. Eymard, T. Gallouët and R. Herbin, A unified approach to mimetic finite difference, hybrid finite volume and mixed finite volume methods. Math. Models Methods Appl. Sci. 20 (2010) 265-295.

[28] M.G. Edwards and C.F. Rogers, A flux continuous scheme for the full tensor pressure equation, in Proc. of the 4th European Conf. on the Mathematics of Oil Recovery. D Røros, Norway (1994).

[29] M.G. Edwards and C.F. Rogers, Finite volume discretization with imposed flux continuity for the general tensor pressure equation. Comput. Geosci. 2 (1998) 259-290.

[30] A. Ern and J.-L. Guermond, Theory and Practice of Finite Elements, Applied Mathematical Sciences 159. Springer-Verlag, New York, NY (2004).

[31] E. Erturk, T.C. Corke and C. Gökçöl, Numerical solutions of 2-D steady incompressible driven cavity flow at high Reynolds numbers. Internat. J. Numer. Methods Fluids 48 (2005) 747-774.

[32] R. Eymard, Th. Gallouët and R. Herbin, The Finite Volume Method, edited by Ph. Charlet and J.L. Lions. North Holland (2000).

[33] R. Eymard, Th. Gallouët and R. Herbin, Discretization of heterogeneous and anisotropic diffusion problems on general nonconforming meshes SUSHI: a scheme using stabilization and hybrid interfaces. IMA J. Numer. Anal. 30 (2010) $1009-1043$. 
[34] R. Eymard, R. Herbin and J.-C. Latché, Convergence analysis of a colocated finite volume scheme for the incompressible Navier-Stokes equations on general 2D or 3D meshes. SIAM J. Numer. Anal. 45 (2007) 1-36.

[35] P. Grisvard, Singularities in Boundary Value Problems. Masson, Paris (1992).

[36] B. Heinrich and K. Pietsch, Nitsche type mortaring for some elliptic problem with corner singularities. Computing 68 (2002) $217-238$.

[37] R. Herbin and F. Hubert, Benchmark on discretization schemes for anisotropic diffusion problems on general grids, in Finite Volumes for Complex Applications V, edited by R. Eymard and J.-M. Hérard. John Wiley \& Sons (2008) 659-692.

[38] R.B. Kellogg, On the Poisson equation with intersecting interfaces. Appl. Anal. 4 (1974/75) 101-129. Collection of articles dedicated to Nikolai Ivanovich Muskhelishvili.

[39] L.S.G. Kovasznay, Laminar flow behind a two-dimensional grid. Proc. Camb. Philos. Soc. 44 (1948) 58-62.

[40] S. Nicaise and A.-M. Sändig, General interface problems. I, II. Math. Methods Appl. Sci. 17 (1994) 395-429, 431-450.

[41] J. Nitsche, On Dirichlet problems using subspaces with nearly zero boundary conditions, in The mathematical foundations of the finite element method with applications to partial differential equations (Proc. Sympos., Univ. Maryland, Baltimore, Md., 1972). Academic Press, New York (1972) 603-627.

[42] R. Stenberg, Mortaring by a method of J.A. Nitsche, in Computational Mechanics: New trends and applications, edited by S.R. Idelsohn, E. Oñate and E.N. Dvorkin. Barcelona, Spain (1998) 1-6. Centro Internacional de Métodos Numéricos en Ingeniería.

[43] R. Temam, Navier-Stokes Equations, Studies in Mathematics and its Applications 2. North-Holland Publishing Co., Amsterdam, revised edition (1979). Theory and numerical analysis, with an appendix by F. Thomasset. 